\title{
Sensitivity study of explosive nucleosynthesis in type Ia supernovae: Modification of individual thermonuclear reaction rates
}

\author{
Eduardo Bravo ${ }^{1, *}$ and Gabriel Martínez-Pinedo ${ }^{2,3, \dagger}$ \\ ${ }^{1}$ Departament de Física i Enginyeria Nuclear, Universidad Politècnica de Catalunya, Carrer Pere Serra 1-15, \\ 08173 Sant Cugat del Vallès, Spain \\ ${ }^{2}$ Technische Universität Darmstadt, Institut für Kernphysik, Schlossgartenstrasse 2, 64289 Darmstadt, Germany \\ ${ }^{3}$ GSI Helmholtzzentrum für Schwerioneneforschung, Planckstrasse 1, 64291 Darmstadt, Germany
}

(Received 20 July 2011; revised manuscript received 10 April 2012; published 18 May 2012)

\begin{abstract}
Background: Type Ia supernovae contribute significantly to the nucleosynthesis of many Fe-group and intermediate-mass elements. However, the robustness of nucleosynthesis obtained via models of this class of explosions has not been studied in depth until now.

Purpose: We explore the sensitivity of the nucleosynthesis resulting from thermonuclear explosions of massive white dwarfs with respect to uncertainties in nuclear reaction rates. We put particular emphasis on indentifying the individual reactions rates that most strongly affect the isotopic products of these supernovae.
\end{abstract}

Method: We have adopted a standard one-dimensional delayed detonation model of the explosion of a Chandrasekhar-mass white dwarf and have postprocessed the thermodynamic trajectories of every mass shell with a nucleosynthetic code to obtain the chemical composition of the ejected matter. We have considered increases (decreases) by a factor of 10 on the rates of 1196 nuclear reactions (simultaneously with their inverse reactions), repeating the nucleosynthesis calculations after modification of each reaction rate pair. We have computed as well hydrodynamic models for different rates of the fusion reactions of ${ }^{12} \mathrm{C}$ and of ${ }^{16} \mathrm{O}$. From the calculations we have selected the reactions that have the largest impact on the supernova yields, and we have computed again the nucleosynthesis using two or three alternative prescriptions for their rates, taken from the JINA REACLIB database. For the three reactions with the largest sensitivity we have analyzed as well the temperature ranges where a modification of their rates has the strongest effect on nucleosynthesis.

Results: The nucleosynthesis resulting from the type Ia supernova models is quite robust with respect to variations of nuclear reaction rates, with the exception of the reaction of fusion of two ${ }^{12} \mathrm{C}$ nuclei. The energy of the explosion changes by less than $\sim 4 \%$ when the rates of the reactions ${ }^{12} \mathrm{C}+{ }^{12} \mathrm{C}$ or ${ }^{16} \mathrm{O}+{ }^{16} \mathrm{O}$ are multiplied by a factor of $\times 10$ or $\times 0.1$. The changes in the nucleosynthesis owing to the modification of the rates of these fusion reactions are also quite modest; for instance, no species with a mass fraction larger than 0.02 experiences a variation of its yield larger than a factor of 2 . We provide the sensitivity of the yields of the most abundant species with respect to the rates of the most intense reactions with protons, neutrons, and $\alpha$. In general, the yields of Fe-group nuclei are more robust than the yields of intermediate-mass elements. Among the species with yields larger than $10^{-8} M_{\odot},{ }^{35} \mathrm{~S}$ has the largest sensitivity to the nuclear reaction rates. It is remarkable that the reactions involving elements with $Z>22$ have a tiny influence on the supernova nucleosynthesis. Among the charged-particle reactions, the most influential on supernova nucleosynthesis are ${ }^{30} \mathrm{Si}+p \rightleftarrows{ }^{31} \mathrm{P}+\gamma,{ }^{20} \mathrm{Ne}+\alpha \rightleftarrows{ }^{24} \mathrm{Mg}+\gamma$, and ${ }^{24} \mathrm{Mg}+\alpha \rightleftarrows{ }^{27} \mathrm{Al}+p$. The temperatures at which a modification of their rate has a larger impact are in the range $2 \lesssim T \lesssim 4 \mathrm{GK}$.

Conclusions: The explosion model (i.e., the assumed conditions and propagation of the flame) chiefly determines the element production of type Ia supernovae and derived quantities such as their luminosity, while the nuclear reaction rates used in the simulations have a small influence on the kinetic energy and final chemical composition of the ejecta. Our results show that the uncertainty in individual thermonuclear reaction rates cannot account for discrepancies of a factor of 2 between isotopic ratios in type Ia supernovae and those in the solar system, especially within the Fe group.

DOI: 10.1103/PhysRevC.85.055805

PACS number(s): 26.30.-k, 24.10.-i, 26.50.+x, 97.60.Bw

\section{INTRODUCTION}

Thanks to their high luminosity, type Ia supernovae (SNIa) are used routinely as standard candles to measure cosmological distances. They are instrumental to our current understanding of the universe, providing evidence for its accelerated expan-

\footnotetext{
*eduardo.bravo@upc.edu

$\dagger$ g.martinez@gsi.de
}

sion [1-6]. Type Ia supernovae play also an important role in the chemical evolution of galaxies, being responsible for most of the Fe-group elements and smaller amounts of silicon, sulfur, argon, and calcium (see, e.g., [7-9]). The elemental composition is evident in optical and infrared spectra recorded from days to months after the explosion (see, e.g., $[10,11])$ and in X-ray spectra of their remnants visible for hundreds of years (for a review, see [12]). Finally, SNIa are one of the key targets for $\gamma$-ray astronomy, as a source of a variety of radioactive isotopes (see, e.g., [13]). 
This is the first paper of a series in which we will study the sensitivity of the nucleosynthesis produced in SNIa with respect to uncertainties in nuclear data. In this paper, we study the sensitivity to variations in rates of thermonuclear reactions (fusion reactions, radiative captures, and transfer reactions). In forthcoming publications, we will study the sensitivity to uncertainties in nuclear masses and in weak interaction rates. Studies of the effect of nuclear data uncertainties in different astrophysical scenarios have been published from time to time during the past few decades (e.g., for example, Refs. [14-21]), to cite only a few, although none of them has dealt with SNIa. These works followed different methodologies to test the impact of nuclear reaction rates. For instance, Ref. [19] varied the rate of individual nuclear reactions relevant for ${ }^{44} \mathrm{Ti}$ nucleosynthesis to determine which reactions were a prime target for the experimental measurement of their cross sections. However, the authors of Ref. [20] designed a numerical experiment to measure the uncertainty of the nucleosynthesis of nova explosions. To this end, they followed a Monte Carlo approach in which they varied simultaneously by random factors all the reaction rates in their network. The focus of this second approach was on the final nova nucleosynthesis rather than in determining the individual reactions that are most influential. Finally, Ref. [21] used theoretical nuclear reaction rates based on four different nuclear mass models to determine their impact on the $r$-process abundances. In this case, the emphasis was on testing different nuclear models. In the present work, we wish to determine the individual nuclear reactions most influential on the nucleosynthesis of SNIa; hence, we follow the same strategy as in Ref. [19].

At present, the favored model of SNIa is the thermonuclear explosion of a carbon-oxygen white dwarf (WD) near the Chandrasekhar mass that accretes matter from a companion star in a close binary system [22]. Other models, such as the sub-Chandrasekhar models or the double degenerate scenario, although not completely ruled out, either have difficulties in explaining the gross features of the spectrum and light curve of normal SNIa, or face severe theoretical objections (see, e.g., Refs. [23-28]). Super-Chandrasekhar models have been proposed to explain a few overluminous SNIa [29-33] but, given the scarcity of observations despite of their high intrinsic luminosity they are thought to represent at most a few percent of all SNIa explosions. Moreover, the properties of the progenitors of super-Chandrasekhar SNIa and the explosions themselves are not well understood. Thus, we concentrate our efforts on the study of a reference SNIa Chandrasekhar-mass model [34].

Even though the hydrostatic evolution of SNIa progenitors lasts for several Gyr while the thermonuclear explosion lasts for a few seconds at most, the outcome is nearly independent of the history of the WD prior to its explosive ignition. This fact is commonly denoted as "stellar amnesia" [35]. The only link between the WD at ignition time and its previous evolution comes through its chemical composition $\left({ }^{12} \mathrm{C},{ }^{16} \mathrm{O},{ }^{22} \mathrm{Ne}\right.$, and other trace species) and the distribution of hot spots that are the seeds of the emerging thermonuclear flame. The influence of uncertain reaction rates, specifically that of the reaction ${ }^{12} \mathrm{C}(\alpha, \gamma){ }^{16} \mathrm{O}$, on the chemical composition of massive WDs has been studied by the authors of Ref. [36], who found that the central $\mathrm{C} / \mathrm{O}$ ratio might vary by a factor of $\sim 13$ at ignition time. However, the effect of different $\mathrm{C} / \mathrm{O}$ ratios on supernova luminosity and nucleosynthesis was studied in Ref. [37], therefore accounting implicitly for a variation on the rate of $\alpha$ capture on ${ }^{12} \mathrm{C}$. They found that the $\mathrm{C} / \mathrm{O}$ ratio can have a sizable impact on the ejecta composition. On the contrary, the authors of Ref. [38] reached the opposite conclusion after analyzing the same problem with their three-dimensional deflagration models of SNIa.

Prior to the SNIa explosion there is a phase of carbon simmering that lasts $\sim 1000 \mathrm{yr}$ and involves temperatures below $10^{9} \mathrm{~K}$. During this phase the neutron excess of matter can be raised owing to electron captures on ${ }^{13} \mathrm{~N}$ and ${ }^{23} \mathrm{Na}$. The leading thermonuclear reactions during carbon simmering are, aside from ${ }^{12} \mathrm{C}+{ }^{12} \mathrm{C}$, reactions that participate in the transmutation of ${ }^{12} \mathrm{C}$ into ${ }^{16} \mathrm{O}:{ }^{12} \mathrm{C}(p, \gamma){ }^{13} \mathrm{~N},{ }^{12} \mathrm{C}(n, \gamma){ }^{13} \mathrm{C}$, and ${ }^{13} \mathrm{C}(\alpha, n){ }^{16} \mathrm{O}$. However, the time scale of neutronization is controlled by the ${ }^{12} \mathrm{C}$ fusion reaction and the rate of electron captures $[39,40]$. Thus, we do not expect that a modification of the rates of radiative captures and transfer reactions can affect appreciably the neutronization of the WD and, hence, the final supernova composition. In this work, we consider only modifications of the thermonuclear reaction rates during the explosive phase of the supernova.

The temperature range relevant for explosive nucleosynthesis in SNIa is approximately $10^{9} \mathrm{~K}$ to $10^{10} \mathrm{~K}$. However, at densities and temperatures in excess of $\sim 10^{8} \mathrm{~g} \mathrm{~cm}^{-3}$ and $\sim 5.5 \times 10^{9} \mathrm{~K}$ nuclei attain a nuclear statistical equilibrium (NSE) state in which the chemical composition, for given temperature, density, and electron mole fraction, is determined by nuclear bulk properties (masses and partition functions); that is, it does not depend on the reaction rates. Under these conditions, NSE erases any imprint of the previous thermodynamic evolution of matter, and reaction rates do not play any role until matter leaves NSE (freeze-out process). The minimum temperature relevant for nucleosynthesis in SNIa depends on the type of combustion front. For a detonation, a shock heats the fuel to temperatures $\gtrsim 2 \times 10^{9} \mathrm{~K}$, the precise value depending mainly on density, before nuclear reactions start modifying the chemical composition. However, the process of combustion within a subsonic flame presents two different phases. Below a critical temperature, $T_{\text {crit }} \sim 2-$ $5 \times 10^{9} \mathrm{~K}$, the matter temperature is set by heat diffusion from the hot ashes, while above $T_{\text {crit }}$ the nuclear energy released by combustion dominates over heat diffusion. Thus, we do not expect modifying the thermonuclear reaction rates below $\sim 1-2 \times 10^{9} \mathrm{~K}$ to have an impact on the final chemical composition.

The structure of the paper is as follows. In the next section, we detail the methodology used to achieve our goals. We describe the postprocessing code used to integrate the nuclear evolutionary equations, the characteristics of our reference SNIa model, the selection of the nuclear reactions to test for variations in their rates, and the ways in which we have modified these rates. In Sec. III, we present the results of the sensitivity study with respect to the fusion reactions of ${ }^{12} \mathrm{C}$, ${ }^{16} \mathrm{O}$, and the $3 \alpha$ reaction, which are the reactions that rule the initial steps of thermonuclear combustion in SNIa. We test modifications of the first two reaction rates for effects on the 
propagation of the flame during a SNIa explosion. In Sec. IV, we present the results of the sensitivity study with respect to thermonuclear reaction rates involving protons, neutrons, and $\alpha$ particles. We have followed different strategies in modifying these reaction rates, using either a fixed enhancement factor or a temperature-dependent one. We have tested as well the use of different prescriptions for the most influential reaction rates, taken from recent literature. For a few reactions we have explored the temperature range where a modification of their rates have a stronger impact on the supernova yields. Finally, in Sec. V, we summarize and give our conclusions.

\section{METHODOLOGY}

\section{A. Integration of the nuclear evolutionary equations}

We have computed the chemical composition of a reference SNIa model with the nucleosynthetic code CRANK (code for the resolution of an adaptive nuclear network). CRANK is a postprocessing code that integrates the temporal evolution of a nuclear network for a given thermal and structural (density) time profile and initial composition. We have selected the nuclear reactions that contribute most to the synthesis of abundant species. Then we recomputed the nucleosynthesis modifying the rate of each one of the selected reactions.

The inputs to CRANK are the nuclear data and the thermodynamic trajectories, as a function of time, of each mass shell of the supernova model. The evolutionary equations for the nuclear composition follow the time evolution of the molar fraction, $Y_{i}$, or abundance of each species until the temperature falls below $10^{8} \mathrm{~K}$, after which time the chemical composition is no longer substantially modified. The nuclear network is integrated with an implicit, iterative method with adaptive time steps. The iterative procedure ends when the molar abundances of all species with $Y_{i}>10^{-14} \mathrm{~mol} \mathrm{~g}^{-1}$ have converged to better than a relative variation of $10^{-6}$.

The nuclear species present in the network are dynamically determined during the calculation. Initially, the network is defined by those species with an appreciable abundance $\left(>10^{-24} \mathrm{~mol} \mathrm{~g}^{-1}\right)$ plus $n, p$, and $\alpha$ 's and the nuclei that can be reached from any of the abundant species by any one of the reactions included in the network. A reaction rate is included in the network only if the predicted change of a molar abundance in the next time step, $\Delta t$, is larger than a threshold:

$$
N_{\mathrm{A}} \rho\langle\sigma v\rangle Y_{i} Y_{j} \Delta t>10^{-20} \mathrm{~mol} \mathrm{~g}^{-1} .
$$

A similar method of integration of the nuclear evolutionary equations using an adaptive network has been described in Ref. [41].

Our nuclear network consists of a maximum of 722 nuclei, from free nucleons up to ${ }^{101} \mathrm{In}$, linked by three fusion reactions $\left(3 \alpha,{ }^{12} \mathrm{C}+{ }^{12} \mathrm{C}\right.$, and $\left.{ }^{16} \mathrm{O}+{ }^{16} \mathrm{O}\right)$, electron and positron captures, $\beta^{-}$and $\beta^{+}$decays, and 12 reactions per each nucleus with $Z \geqslant 6$ : $(n, \gamma),(n, p),(n, \alpha),(p, \gamma),(p, n)$, $(p, \alpha),(\alpha, \gamma),(\alpha, n),(\alpha, p),(\gamma, n),(\gamma, p)$, and $(\gamma, \alpha)$. We show the nuclear network in Table I. From the whole set of reactions that might be included in the calculations, only 3138 enter effectively into the reaction network equations during the integration of the thermodynamic trajectories in our SNIa model.

The thermonuclear reaction rates, nuclear masses, and partition functions are taken from the REACLIB compilation [42]. Both theoretical and experimental thermonuclear reaction rates are fitted in the $\mathrm{JINA}^{1}$ REACLIB library by an analytic function with seven parameters. The fits are usually better than $5 \%$ although deviations up to $30 \%$ are possible. The authors estimate an additional uncertainty typically of order $30 \%$ in the original reaction rates. Electron screening to thermonuclear reactions in the strong, intermediate, and weak regimes was taken into account $[43,44]$. In general, in the conditions achieved during thermonuclear supernova explosions the electron screening factors are small [45]. Weak interaction rates were taken from Refs. [46,47].

\section{B. Type Ia supernova model}

Our reference SNIa model is the one-dimensional delayeddetonation model DDTc in Ref. [48], characterized by its deflagration-to-detonation (DDT) transition density, $\rho_{\mathrm{DDT}}=$ $2.2 \times 10^{7} \mathrm{~g} \mathrm{~cm}^{-3}$. The supernova progenitor is a Chandrasekhar mass WD of central density $1.8 \times 10^{9} \mathrm{~g} \mathrm{~cm}^{-3}$ and uniform composition: $49.5 \%{ }^{12} \mathrm{C}, 49.5 \%{ }^{16} \mathrm{O}$, and $1 \%$ ${ }^{22} \mathrm{Ne}$ by mass. In this model the flame begins as a subsonic deflagration flame near the center of the star. As the flame propagates through the star, the pressure rises and the star expands. When the flame reaches a zone with a low-enough density, $\rho_{\mathrm{DDT}}$, there is a transition to a supersonic detonation that burns most of the remaining fuel. Finally, the nuclear energy released is enough to unbind the whole star and eject its matter into the interstellar medium. In Fig. 1 we show the profiles of the most relevant physicochemical quantities affecting the nucleosynthesis.

This kind of SNIa model generates a layered structure (see Fig. 2) in which the inner several tenths of a solar mass achieve maximum temperatures high enough $\left(T_{\max } \gtrsim 5.5 \times 10^{9} \mathrm{~K}\right)$ to process matter into NSE, undergoing copious electron captures. When matter expands the composition is relaxed out of NSE and consists mainly of iron group elements with isotopic fractions determined by the electron mole number resulting from the electron captures phase. As can be seen in Fig. 1, in our reference model the electron captures modify the progenitor electron mole number only in the central $\sim 0.1 M_{\odot}$. The zone where the transition from deflagration to detonation takes place, at a mass coordinate of $\sim 0.2 M_{\odot}$, can be identified by the trough in the $Y_{\mathrm{p}}, Y_{\alpha}$, and $Y_{\mathrm{n}}$ profiles. The central $0.4 M_{\odot}$ reach NSE, from which roughly $0.24 M_{\odot}$ experience a moderately $\alpha$-rich freeze-out. Shortly after the detonation forms, it propagates fast through the WD, which has no time to relax its structure before the combustion front burns most of the remaining fuel (this condition can be identified in Fig. 1 by the crowding of the star symbols between $T_{9} \sim 4$ and $\sim 5.5$ ). Between Lagrangian mass coordinates of $\sim 0.4 M_{\odot}$ and $\sim 1.1 M_{\odot}$ the peak temperatures and densities are high enough to experience Si burning and achieve quasistatistical

\footnotetext{
${ }^{1}$ http://groups.nscl.msu.edu/jina/reaclib/db/.
} 
TABLE I. Nuclear network.

\begin{tabular}{|c|c|c|c|c|c|c|c|c|c|c|c|}
\hline$n$ & 1 & 1 & $\mathrm{Al}$ & 22 & 36 & $\mathrm{Fe}$ & 49 & 63 & $\mathrm{Y}$ & 79 & 101 \\
\hline $\mathrm{H}$ & 1 & 4 & $\mathrm{Si}$ & 24 & 38 & Co & 51 & 65 & $\mathrm{Zr}$ & 81 & 101 \\
\hline $\mathrm{Li}$ & 4 & 11 & $\mathrm{~S}$ & 28 & 42 & $\mathrm{Cu}$ & 55 & 71 & Mo & 87 & 101 \\
\hline $\mathrm{Be}$ & 6 & 14 & $\mathrm{Cl}$ & 30 & 44 & $\mathrm{Zn}$ & 57 & 78 & $\mathrm{Tc}$ & 89 & 101 \\
\hline B & 7 & 17 & $\mathrm{Ar}$ & 32 & 46 & $\mathrm{Ga}$ & 61 & 81 & $\mathrm{Ru}$ & 91 & 101 \\
\hline $\mathrm{N}$ & 10 & 21 & $\mathrm{Ca}$ & 36 & 51 & As & 65 & 85 & $\mathrm{Pd}$ & 95 & 101 \\
\hline $\mathrm{O}$ & 12 & 23 & $\mathrm{Sc}$ & 38 & 52 & $\mathrm{Se}$ & 67 & 87 & $\mathrm{Ag}$ & 97 & 101 \\
\hline $\mathrm{F}$ & 14 & 25 & $\mathrm{Ti}$ & 40 & 54 & $\mathrm{Br}$ & 69 & 90 & $\mathrm{Cd}$ & 99 & 101 \\
\hline $\mathrm{Ne}$ & 16 & 27 & $\mathrm{~V}$ & 42 & 56 & $\mathrm{Kr}$ & 71 & 93 & In & 101 & 101 \\
\hline $\mathrm{Na}$ & 18 & 34 & $\mathrm{Cr}$ & 44 & 58 & $\mathrm{Rb}$ & 73 & 99 & & & \\
\hline $\mathrm{Mg}$ & 20 & 35 & $\mathrm{Mn}$ & 46 & 60 & $\mathrm{Sr}$ & 77 & 100 & & & \\
\hline
\end{tabular}

equilibrium (QSE) of the Fe group, although this group does not achieve equilibrium with the Si group. Farther out from the center, a tinier amount of mass is subject to explosive oxygen and neon burning, and only a few thousandths of a solar mass experience only explosive carbon burning. The mass of unburned carbon ejected by the supernova explosion is on the same order, in agreement with the upper limits deduced in Ref. [49]. We note that all the nucleosynthetic processes deemed relevant in SNIa feature in our reference model.

For reference, we give in Table II the nucleosynthesis obtained for this supernova model. The composition given in this and forthcoming tables corresponds to a time of one day after beginning of the explosion; hence, there appear radioactive as well as stable nuclides. We have included in this table all nuclides whose ejected mass is $m_{i}>10^{-5} M_{\odot}$, with the exception of ${ }^{26} \mathrm{Al}$, which has been included because it is an interesting radionuclide. The ejected mass of ${ }^{56} \mathrm{Ni}$ is $0.675 M_{\odot}$, and the kinetic energy of the ejecta is $1.16 \times 10^{51}$ erg, both values deemed typical for normal bright SNIa. The resulting chemical composition (Fig. 2) compares well with the abundance stratification induced from observations of normal SNIa as, for instance, SN2003du (e.g., Fig. 8 in Ref. [50], where it was estimated that the ejected mass of ${ }^{56} \mathrm{Ni}$ was $\left.0.65 M_{\odot}\right)$. Model DDTc also provides an excellent match to the
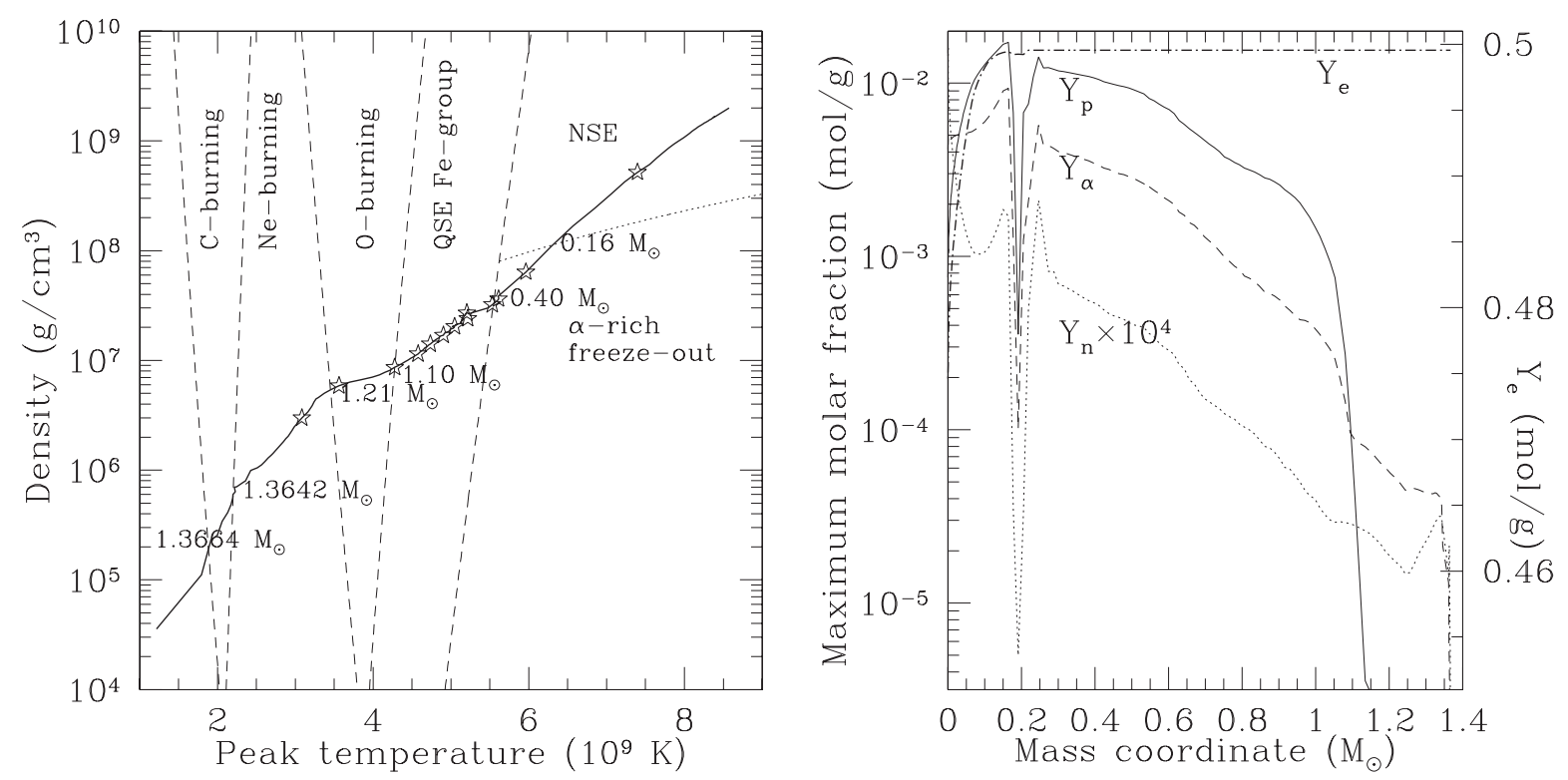

FIG. 1. Profiles of physicochemical properties across the reference model, as a function of the Lagrangian mass coordinate (zero at the center). (Left) Peak temperature and density achieved at each mass shell during the supernova explosion (thick solid line). Star marks have been located every $0.1 M_{\odot}$, with the center of the WD at the top right end of the solid line and the surface at its bottom left end. The $\rho-T$ plane has been divided according to approximate locations of different explosive nucleosynthetic processes (dashed and dotted lines). We indicate as well the Lagrangian mass coordinate at which the solid line crosses the dashed and dotted lines. (Right) Maximum molar fractions of neutrons, protons, and $\alpha$ 's achieved at a given mass coordinate at any time during the explosion and final electron mole number (dot-dashed line). Note that the neutron molar fraction has been scaled up by a factor of $10^{4}$ for presentation purposes. 
TABLE II. Nucleosynthesis of the reference type Ia supernova model.

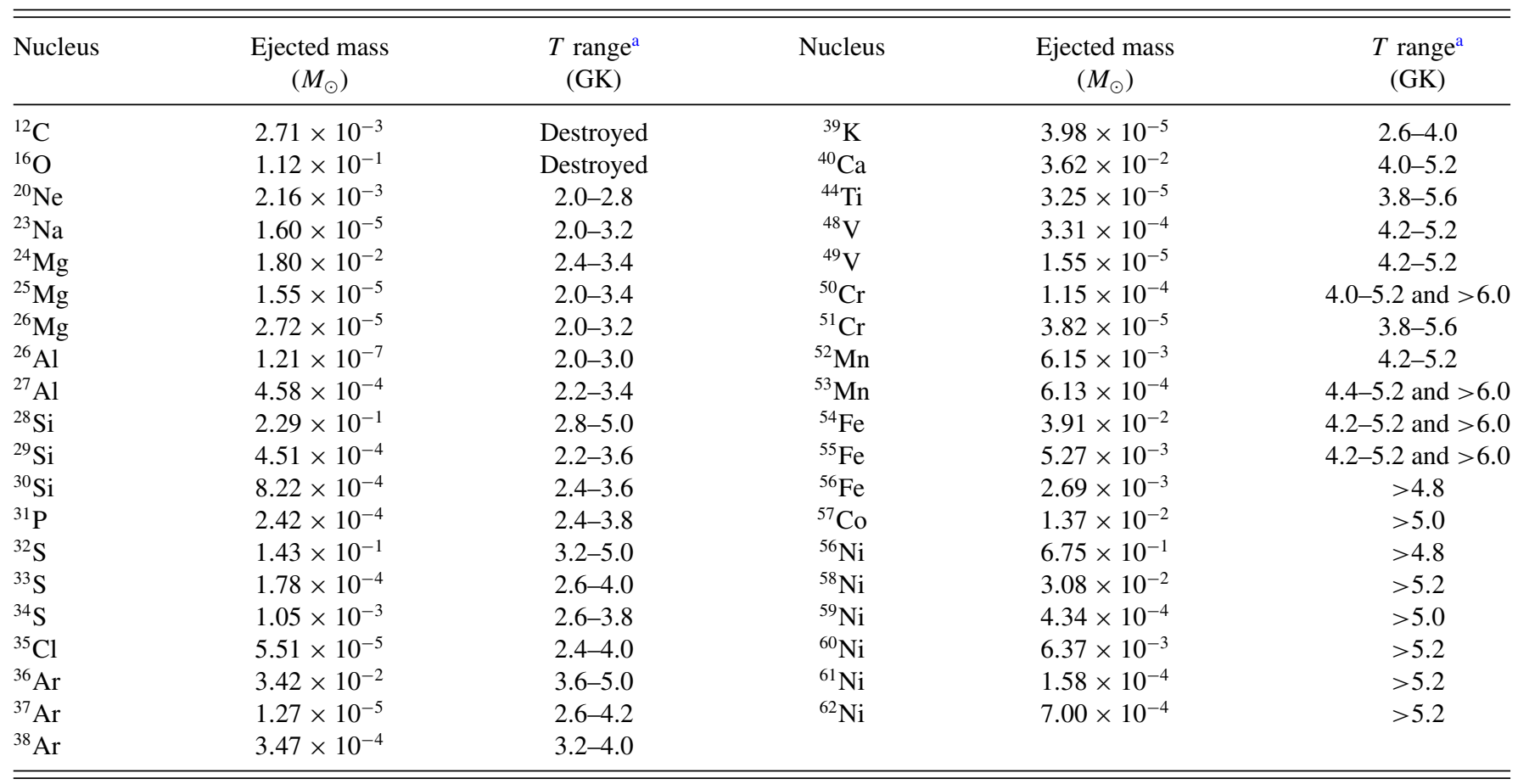



X-ray spectrum of the remnant of SN1572 (Tycho), a prototype of SNIa (see Fig. 7 in Ref. [51]).

The maximum abundances of free protons, neutrons, and $\alpha$ particles attained during the explosion are shown in the right panel of Fig. 1, as a function of the Lagrangian mass coordinate within the exploding WD. These profiles can be used to gain insight into the expected sensitivities of the nucleosynthesis with respect to different types of nuclear reactions, to be

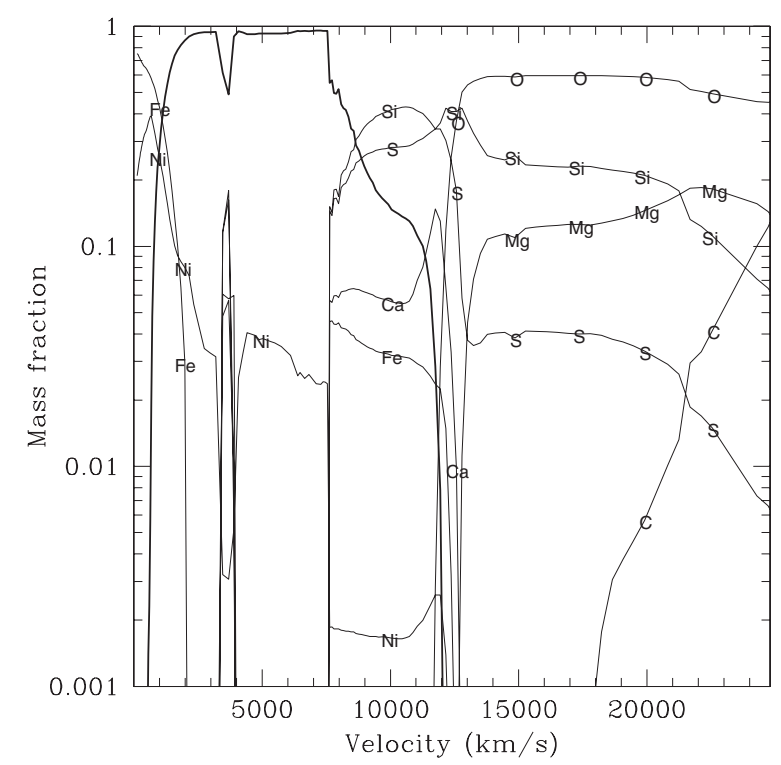

FIG. 2. Chemical composition of the reference model as a function of the final velocity. The curves labeled as $\mathrm{Fe}$ and $\mathrm{Ni}$ include only stable isotopes. The thick curve is the mass fraction of ${ }^{56} \mathrm{Ni}$. discussed in the next sections. Neutrons are always the less abundant nucleons by $\sim 5-6$ orders of magnitude; thus, we expect that the nucleosynthesis will not be too sensitive to reactions with neutrons, except, perhaps, in the outer $\sim 0.1 M_{\odot}$. Note that neutrons are relatively abundant in the very center of the WD, because of the lower $Y_{\mathrm{e}}$ that results from efficient electron captures in NSE matter at high density, but nucleosynthesis in these layers is not expected to be sensitive to the rate of any particular reaction with neutrons because the chemical composition there is controlled by the Saha equation until matter cools to low temperatures. Protons and $\alpha$ particles have similar abundances within the inner $\sim 1.1 M_{\odot}$, although their maximum molar fractions decrease steadily outward within the detonated matter $\left(M \gtrsim 0.25 M_{\odot}\right)$. Beyond $\sim 1.1 M_{\odot}$, the maximum abundance achieved by protons is much lower than that of $\alpha$ particles. The maximum temperatures attained in these layers stay below $\sim 4.4 \times 10^{9} \mathrm{~K}$, implying that the thermonuclear combustion hardly goes beyond $\mathrm{O}$ burning. Thus, we expect that the products of $\mathrm{O}$ burning will be mostly sensitive to reactions with $\alpha$ particles.

The above analysis can be complemented with an examination of the molar fluxes owing to different reaction types [e.g., $(p, n)$, etc.]. In Fig. 3 we show the evolution of the net molar fluxes in two representative mass shells of our SNIa model, grouped by reaction type. The net molar fluxes of a given reaction type in a mass shell are accumulated in time according to

$$
\sum\left[\int \rho N_{\mathrm{A}}\langle\sigma v\rangle_{j k} Y_{j} Y_{k} \mathrm{~d} t\right],
$$

where $\rho$ is density, $N_{\mathrm{A}}$ is Avogadro's number, $Y_{j}$ is the molar fraction of species $j$, and the time integral extends from 

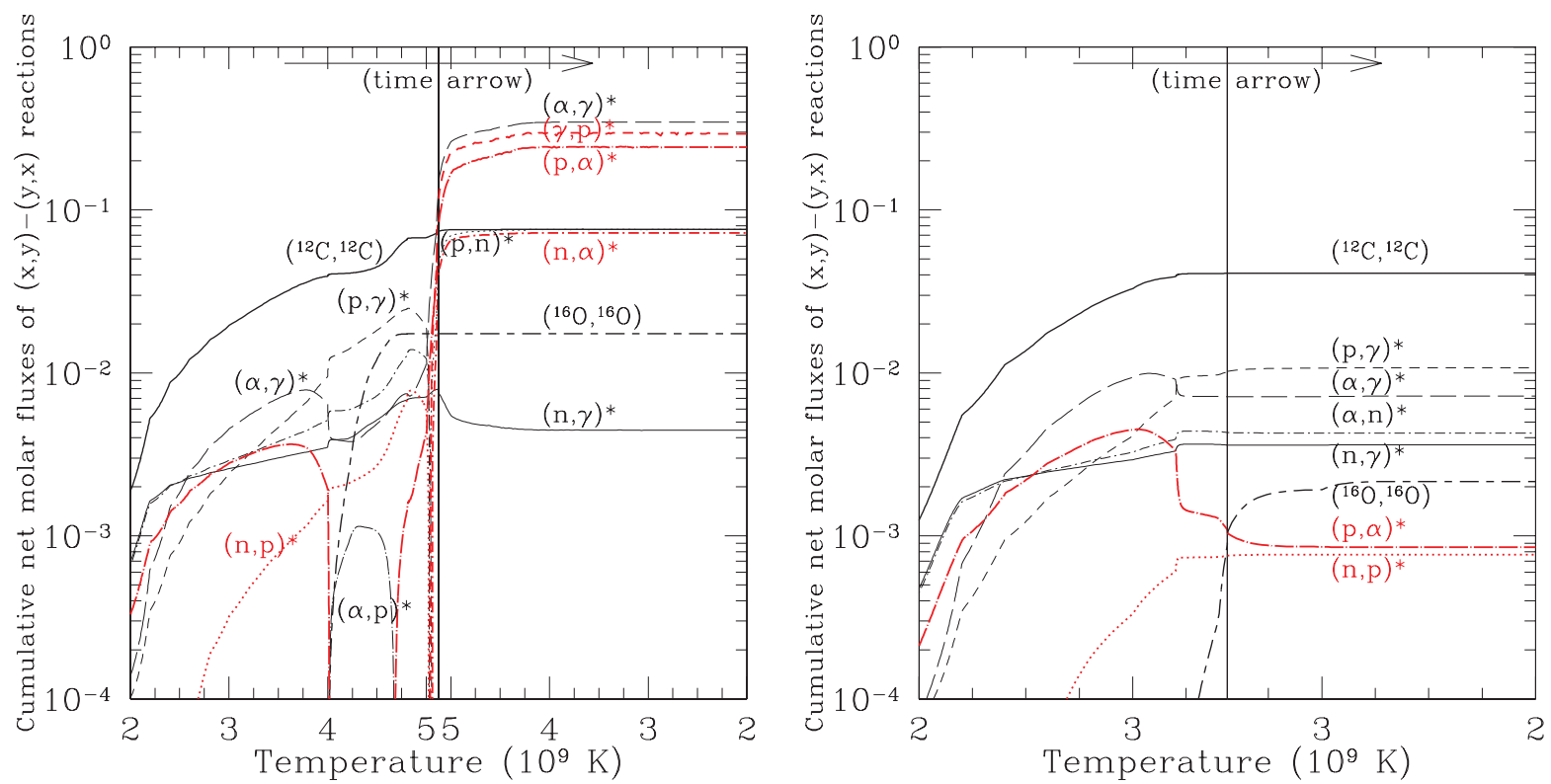

FIG. 3. (Color online) Cumulative net molar fluxes of direct reactions minus inverse reactions, grouped by reaction type, compared to the ${ }^{12} \mathrm{C}$-fusion and ${ }^{16} \mathrm{O}$-fusion reaction fluxes, as functions of temperature. Each reaction type is identified by a different line type and a label; in each label an $*$ is drawn to recall that the fluxes take into account direct and inverse reactions. Thin (black) curves represent those cases in which the (accumulated) molar flux of the direct reaction is larger than that of the inverse reaction, while thick (red) curves belong to the opposite case. Vertical lines are drawn to separate the heating phase from the cooling phase. (Left) Evolution at Lagrangian mass coordinate of $0.5 M_{\odot}$. (Right) Evolution at Lagrangian mass coordinate of $1.2 M_{\odot}$.

thermal runaway until the temperature goes below $10^{8} \mathrm{~K}$. The summation extends to all reactions of the given type, from which their inverse reactions are subtracted, for example, in the computation of the net molar fluxes of the $(p, n)$-type reactions all the $(n, p)$ reactions are considered inverse reactions and their contributions are deducted from those of the direct $(p, n)$ reactions.

In the left panel of Fig. 3 we show the net molar fluxes in a mass shell located at a Lagrangian mass coordinate of $0.5 M_{\odot}$. This layer was hit by the detonation wave $\sim 2.5 \mathrm{~s}$ after central thermal runaway, when its density was $1.17 \times$ $10^{7} \mathrm{~g} \mathrm{~cm}^{-3}$, and heated to $\gtrsim 2 \times 10^{9} \mathrm{~K}$ by the shock front associated with the detonation. Above this temperature, it is the energy release by nuclear reactions that controls the evolution of temperature. The temperature rises very fast at the beginning owing to rapid burning of carbon and oxygen, mainly to produce silicon and sulfur. About $1 \mathrm{~ms}$ after being shocked, a maximum temperature of $5.12 \times 10^{9} \mathrm{~K}$ is achieved. Later, matter expands and cools with a longer time scale (it takes $0.1 \mathrm{~s}$ to cool by $2 \times 10^{9} \mathrm{~K}$ ), while most of the nuclear reactions are nearly in equilibrium with their inverse reactions. During the heating phase, it is the ${ }^{12} \mathrm{C}+{ }^{12} \mathrm{C}$ reaction which dominates the nuclear fluxes, followed by radiative captures of protons and $\alpha$ particles once the temperature exceeds $\sim 2.5 \times 10^{9} \mathrm{~K}$. Compared to the plethora of reactions with light particles unleashed by the carbon fusion reaction, the contribution of the ${ }^{16} \mathrm{O}+{ }^{16} \mathrm{O}$ reaction is quite modest until the temperature exceeds $\gtrsim 4 \times 10^{9} \mathrm{~K}$. Above $\sim 5 \times 10^{9} \mathrm{~K}$ there is a sharp increase in the cumulative molar fluxes belonging to $(\alpha, \gamma),(\gamma, p)$, and $(p, \alpha)$ reactions, which reach similar levels.
However, during the cooling phase there is little additional contribution to the net molar fluxes, and $(p, n)$ and $(n, \alpha)$ reactions attain a level similar to that of ${ }^{16} \mathrm{O}+{ }^{16} \mathrm{O}$, while $(n, \gamma)$ reactions are the ones that process the smallest mass. Note that, in this mass shell, the final cumulative molar flux owing to the ${ }^{16} \mathrm{O}$ fusion reaction is about a factor of 4 smaller than that owing to the ${ }^{12} \mathrm{C}$ fusion reaction. Taking this mass shell as representative of layers that experience incomplete $\mathrm{Si}$ burning, we expect that the products of this nucleosynthetic process will be most sensitive to $(\alpha, \gamma),(\gamma, p)$, and $(p, \alpha)$ reactions and their inverses. Note that in shells that achieve a temperature high enough to reach NSE all the molar fluxes established prior to NSE are irrelevant, because NSE erases all memory of previous nuclear processes with the exception of weak interactions.

The right panel of Fig. 3 shows the net molar fluxes in a mass shell located at a Lagrangian mass coordinate of $1.2 M_{\odot}$. In this case the maximum temperature achieved was $3.45 \times 10^{9} \mathrm{~K}$, because the density at the time of detonation impact (at $t \sim$ $2.64 \mathrm{~s}$ ) was only $2.4 \times 10^{6} \mathrm{~g} \mathrm{~cm}^{-3}$. Owing to the small value of the maximum temperature, the ${ }^{12} \mathrm{C}+{ }^{12} \mathrm{C}$ reaction dominates the molar fluxes at all times. Oxygen burning is incomplete, the final molar flux owing to the ${ }^{16} \mathrm{O}$ fusion reaction being about 20 times smaller than that owing to the ${ }^{12} \mathrm{C}$ fusion reaction. Even $(n, \gamma)$ reactions process more matter than the ${ }^{16} \mathrm{O}$ fusion reaction. Taking this mass shell as representative of layers that do not go beyond carbon burning, we expect that the products of this nucleosynthetic process will be most sensitive to the rate of the ${ }^{12} \mathrm{C}+{ }^{12} \mathrm{C}$ reaction and, to a lesser extent, to $(p, \gamma)$ and $(\alpha, \gamma)$ reactions. 


\section{Selection of the nuclear reactions}

As explained before, only 3138 nuclear reactions exceed the threshold of Eq. (1) and are actually included in the nucleosynthesis calculation. However, most of these reactions contribute negligibly to the determination of the final chemical composition of the supernova ejecta. To determine the most relevant reactions, we define the total mass processed by a nuclear reaction, between particle $k$ and nucleus $j$, in all the mass shells of the supernova model $\mathfrak{M}_{j k}$ :

$$
\mathfrak{M}_{j k}=\sum_{\alpha}\left[M_{\alpha} \int \rho\langle\sigma v\rangle_{j k} N_{\mathrm{A}} Y_{j} Y_{k}\left(A_{j}+A_{k}\right) \mathrm{d} t\right],
$$

where $M_{\alpha}$ is the mass of shell $\alpha$ of the supernova model and $A_{j}$ is the baryon number of species $j$. In the computation of the integral we have not taken into account reactions above $5 \times 10^{9} \mathrm{~K}$, because at such temperatures the direct and inverse reactions are in equilibrium, causing the nuclear abundances to be determined by properties of the nuclei involved (mass, partition function) instead of the reaction rates. For mass shells that went through NSE, the computation of the integral in Eq. (3) starts when the temperature drops below $5 \times$ $10^{9} \mathrm{~K}$, because their chemical composition is insensitive to the nuclear history prior to the NSE state (with the exception of weak interactions, whose effect is not addressed in the present work).

The reactions we have selected for careful study are the three fusion reactions plus those for which $\mathfrak{M}_{j k} \geqslant 10^{-8} M_{\odot}$. This warrants that we test all the reactions able to contribute significantly to the synthesis of every species whose yield is larger than the chosen $10^{-8} M_{\odot}$. Each time we integrate the nuclear evolutionary equations we modify by the same factor the direct and inverse reactions. Following this procedure, we find that the nucleosynthesis at this chosen level could be sensitive to 1096 (pairs of) reactions in addition to the abovementioned three fusion reactions.

Table III gives the masses processed by the three fusion reactions and the top ten radiative captures and transfer reactions, where the masses processed by the inverse reactions have been subtracted from those of the direct reactions. The quoted values of $\mathfrak{M}_{j k}$ give a quite generous upper limit of the impact these reactions might have on the resulting nucleosynthesis of the supernova, as the subsequent nuclear reactions destroy the products of earlier reactions. As we see in the following, the top ten reactions listed in Table III are not, in fact, the most influential reactions.

\section{Modification of the reaction rates}

As a first approach to studying the sensitivity to the different reaction rates, we modify them, one by one, by a fixed factor, equal to either $f_{0}=10$ or $f_{0}=0.1$, repeating the nucleosynthesis calculation for each variation. As mentioned previously, each time we modify the rate of a reaction we modify as well by the same factor the rate of the inverse reaction, to maintain detailed balance.

The Gamow energies in the reactions that play a significant role in the nucleosynthesis of type Ia supernovae go from a few tenths of a MeV (for instance, $E_{0}=0.39 \mathrm{MeV}$ for the
${ }^{12} \mathrm{C}+p \rightleftarrows{ }^{13} \mathrm{~N}+\gamma$ reaction at $T=10^{9} \mathrm{~K}$ ) to nearly $10 \mathrm{MeV}$ (e.g., $E_{0}=8.50 \mathrm{MeV}$ for the ${ }^{62} \mathrm{Zn}+\alpha \rightleftarrows{ }^{65} \mathrm{Ga}+p$ reaction at $T=5 \times 10^{9} \mathrm{~K}$ ). It is expected, from both theoretical and experimental arguments, that the uncertainties in the rates at low temperatures are larger than at high temperatures [52]. Most of the theoretical reaction rates we have used are based on an statistical model of nuclei, which assumes formation of a compound nucleus with a high-level density, a condition generally satisfied at high temperatures. Furthermore, experimental measurements of nuclear cross sections involving high- $Z$ nuclei are generally difficult to perform at energies below the Coulomb barrier. Consequently, we use a second approach in which the reaction rates are modified by applying a factor that is a monotonic decreasing (exponential) function of the temperature. We have applied the following temperature-dependent factor to each reaction rate:

$$
f(T)=1+\left(f_{0}-1\right) \exp \left(-\frac{T}{3 \times 10^{9} \mathrm{~K}}\right),
$$

where $f_{0}=10$ or 0.1 is the fixed factor applied in the first approach. Of course, we are not trying to convey that Eq. (4) is representative of the uncertainty of all the reactions studied here (see Secs. IV C and IV D), but it provides a convenient way to investigate the effects of a temperature-dependent rate error.

\section{SENSITIVITY TO THE RATE OF FUSION OF CARBON AND OF OXYGEN AND THE TRIPLE- $\alpha$ REACTION}

We have checked the effect of varying each fusion reaction rate by the factors given above, either taking them fixed or as function of the temperature. Because the fusion reactions are relevant for the nuclear-energy generation in the supernova explosion, we have recomputed the hydrodynamics with the modified reaction rates and give the results in Sec. III A. When we kept unchanged the thermodynamic trajectories of the reference model, but the reaction rates were modified in the nucleosynthetic code, we obtained the results shown in Sec. III B.

\section{A. Rate modified in the hydrodynamic explosion model}

The nuclear-energy release of the supernova is more sensitive to the rate of the ${ }^{16} \mathrm{O}$ fusion reaction than to that of ${ }^{12} \mathrm{C}$. The final kinetic energy of the ejecta varies by less than $1 \%$ when the ${ }^{12} \mathrm{C}+{ }^{12} \mathrm{C}$ reaction rate is varied by a factor of 10 or 0.1 , either fixed or as a function of the temperature given by Eq. (4). In contrast, the same relative variation in the rate of the ${ }^{16} \mathrm{O}+{ }^{16} \mathrm{O}$ reaction produces a change of kinetic energy of up to $\pm 4 \%$. We ascribe this lack of sensitivity to the relatively small amount of mass that does not experience complete carbon or oxygen burning. Figure 4 shows the final chemical profiles in the outermost $0.2 M_{\odot}$ of ejecta, where the changes in the ${ }^{12} \mathrm{C}+{ }^{12} \mathrm{C}$ and the ${ }^{16} \mathrm{O}+{ }^{16} \mathrm{O}$ reaction rates are most influential. The three panels show the profiles belonging to our reference model and the models in which either the carbon or the oxygen fusion rates are increased by a factor of 10 , both in the hydrodynamic as well as in the nucleosynthetic 
TABLE III. Masses processed by the fusion reactions and the top ten radiative captures and transfer reactions.

\begin{tabular}{lccc}
\hline \hline Reaction & $\mathfrak{M}_{j k}\left(M_{\odot}\right)$ & Reaction & $\mathfrak{M}_{j k}\left(M_{\odot}\right)$ \\
\hline${ }^{12} \mathrm{C}+{ }^{12} \mathrm{C}$ & 0.524 & ${ }^{29} \mathrm{Si}+\alpha \rightleftarrows{ }^{32} \mathrm{~S}+n$ & 0.70 \\
${ }^{16} \mathrm{O}+{ }^{16} \mathrm{O}$ & 0.198 & ${ }^{30} \mathrm{P}+\alpha \rightleftarrows{ }^{33} \mathrm{~S}+p$ & 0.68 \\
${ }^{3} \alpha$ & $6.7 \times 10^{-4}$ & ${ }^{32} \mathrm{Si}+p \rightleftarrows \alpha \rightleftarrows{ }^{30} \mathrm{P}+\gamma$ & $\mathrm{Cl}+p$ \\
${ }^{28} \mathrm{Si}+\alpha \rightleftarrows{ }^{32} \mathrm{~S}+\gamma$ & 0.93 & ${ }^{33} \mathrm{~S}+\alpha \rightleftarrows{ }^{36} \mathrm{Ar}+n$ & 0.67 \\
${ }^{28} \mathrm{Si}+p \rightleftarrows{ }^{29} \mathrm{P}+\gamma$ & 0.84 & ${ }^{27} \mathrm{Al}+\alpha \rightleftarrows{ }^{30} \mathrm{Si}+p$ & 0.65 \\
${ }^{29} \mathrm{P}+\alpha \rightleftarrows{ }^{32} \mathrm{~S}+p$ & 0.83 & & 0.64 \\
${ }^{28} \mathrm{Si}+\alpha \rightleftarrows{ }^{31} \mathrm{P}+p$ & 0.77 & & 0.63 \\
\hline \hline
\end{tabular}

codes. As can be seen, increasing the ${ }^{12} \mathrm{C}+{ }^{12} \mathrm{C}$ rate by a factor of 10 barely affects the limits of the region undergoing carbon burning, which move outward $\sim 0.004 M_{\odot}$. However, when the ${ }^{16} \mathrm{O}+{ }^{16} \mathrm{O}$ reaction rate is enhanced by the same factor the limits of the oxygen-burning region move outward $\sim 0.082 M_{\odot}$. We conclude that the impact of the rates uncertainties on the energy of the supernova is negligible.

Figures 5 to 8 and Table IV show the impact of the changes in the fusion rates of ${ }^{12} \mathrm{C}$ and of ${ }^{16} \mathrm{O}$ on the nucleosynthesis of the type Ia supernova when we modified the rates in the full supernova simulation. Figures 5 and 6 show the results sorted by final mass fraction of the product species. The mass fractions of the most abundant species are insensitive to the rate of fusion of ${ }^{12} \mathrm{C}$. As one goes to smaller abundances, the scatter of the yield ratio is larger. Among the species with mass fraction greater than 0.01 , there is only one nuclide that is significantly affected by the modification of the rate of ${ }^{12} \mathrm{C}+{ }^{12} \mathrm{C}$ : Not surprisingly, it is ${ }^{24} \mathrm{Mg}$. When the factor that modifies the ${ }^{12} \mathrm{C}$ fusion rate is a function of temperature [Eq. (4)] the effect on the yields of all species is dramatically

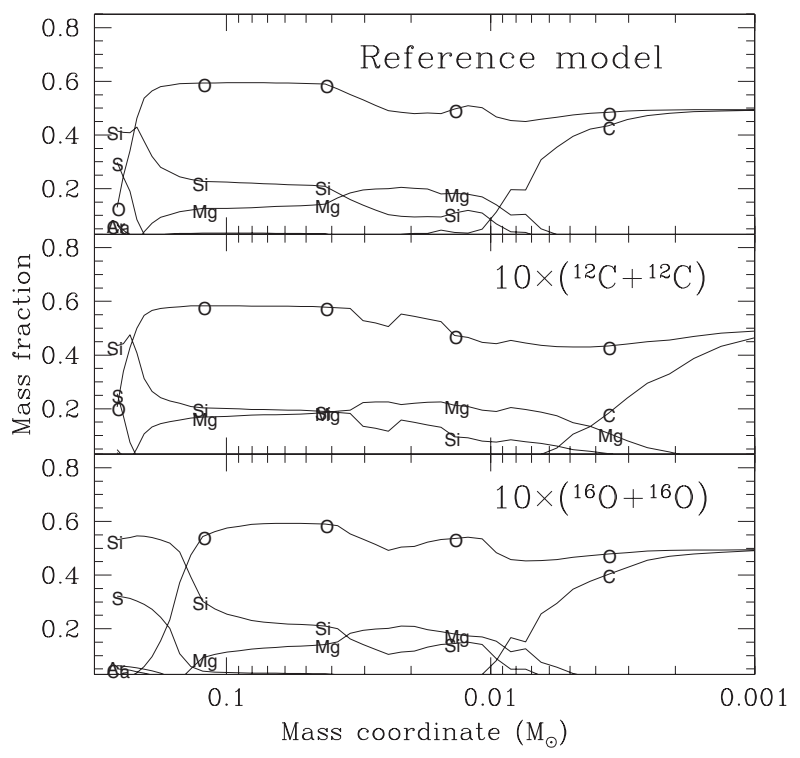

FIG. 4. Final chemical profile within the outer layers of the SNIa ejecta for three of the computed models: our reference model (top), the model with the ${ }^{12} \mathrm{C}+{ }^{12} \mathrm{C}$ reaction increased by a constant factor of 10 (middle), and the model with the ${ }^{16} \mathrm{O}+{ }^{16} \mathrm{O}$ reaction increased by a constant factor of 10 (bottom). In this plot, the mass coordinate is zero at the WD surface and increases inward. reduced (bottom frame in Fig. 5): No species experiences an increase larger than a factor of 2 in its abundance, and only a few species with quite small mass fractions $\left(X_{i}<10^{-6}\right)$ experience a reduction of more than a factor of 2 in their yields when the ${ }^{12} \mathrm{C}$ fusion reaction rate is multiplied by a factor of 10 .

When the ${ }^{16} \mathrm{O}+{ }^{16} \mathrm{O}$ rate is modified (Fig. 6) the impact is in general smaller than when the ${ }^{12} \mathrm{C}$ fusion rate was modified. However, many of the most abundant species are more sensitive to the ${ }^{16} \mathrm{O}$ fusion rate than to the ${ }^{12} \mathrm{C}$ rate because the products of $\mathrm{C}$ burning (mainly ${ }^{16} \mathrm{O},{ }^{20} \mathrm{Ne}$, and ${ }^{24} \mathrm{Mg}$ ) are, in general, less abundant than the products of $\mathrm{O}$ burning (mainly ${ }^{28} \mathrm{Si},{ }^{32} \mathrm{~S},{ }^{36} \mathrm{Ar}$, and ${ }^{40} \mathrm{Ca}$ ).

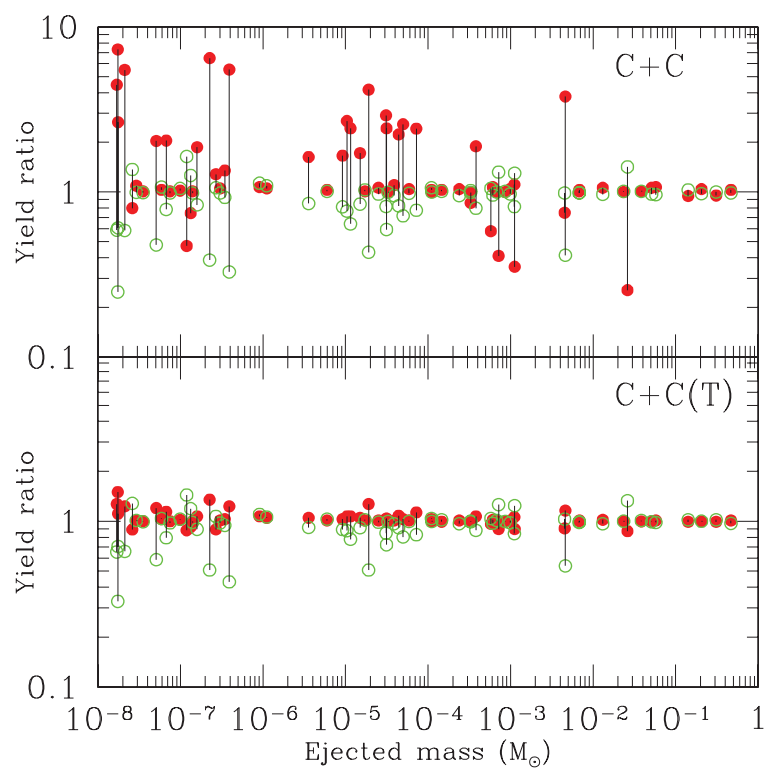

FIG. 5. (Color online) Ratio of mass ejected for each nuclide with a modified ${ }^{12} \mathrm{C}+{ }^{12} \mathrm{C}$ reaction rate with respect to the mass ejected in the reference model, as a function of the mass fraction in the reference model (most abundant species are located to the right of each figure). Note that the species included in Table IV are those with an ejected mass larger than $10^{-5} M_{\odot}$. Vertical lines link the results obtained for the same nuclide when the rate is either increased or decreased. The reaction rate was modified both in the hydrodynamics calculation as well as in the nucleosynthetic code. (Top) Rate multiplied by a fixed factor, either $\times 10$ (green open circles) or $\times 0.1$ (red solid circles). (Bottom) Rate multiplied by a factor function of temperature given by Eq. (4), with either $f_{0}=10$ (green open circles) or $f_{0}=0.1$ (red solid circles). 


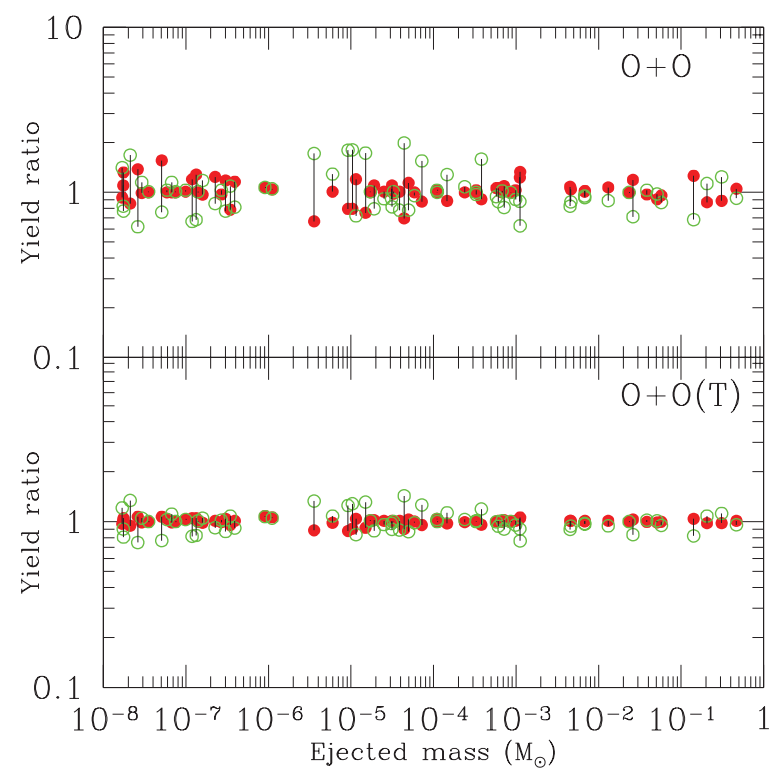

FIG. 6. (Color online) Ratio of mass ejected for each nuclide with a modified ${ }^{16} \mathrm{O}+{ }^{16} \mathrm{O}$ reaction rate with respect to the mass ejected in the reference model, as a function of the mass fraction in the reference model (most abundant species are located to the right of each figure). Note that the species included in Table IV are those with an ejected mass larger than $10^{-5} M_{\odot}$. Vertical lines link the results obtained for the same nuclide when the rate is either increased or decreased. We modified the reaction rate both in the hydrodynamics calculation and in the nucleosynthetic code. (Top) Rate multiplied by a fixed factor, either $\times 10$ (green open circles) or $\times 0.1$ (red solid circles). (Bottom) Rate multiplied by a factor function of temperature given by Eq. (4), with either $f_{0}=10$ (green open circles) or $f_{0}=0.1$ (red solid circles).

Figure 7 presents the same results as Fig. 5 from another perspective: The impact of the modification of the ${ }^{12} \mathrm{C}+{ }^{12} \mathrm{C}$ reaction rate is shown against the element atomic number. The trend that can be observed in this figure is that increasing the ${ }^{12} \mathrm{C}$ fusion rate (green open circles) decreases the abundances both of CNO nuclei and of intermediate-mass elements (IMEs) between phosphorus and titanium and increases the abundances of magnesium, aluminum, and silicon, while elements beyond vanadium are scarcely affected at all. If the rate of ${ }^{12} \mathrm{C}$ fusion is decreased (red solid circles) the trend is inverted, but the yields are, in general, more sensitive to a decrease in this rate than to an increase by the same factor.

As can be seen in Fig. 8, an increase in the ${ }^{16} \mathrm{O}+{ }^{16} \mathrm{O}$ reaction rate results in a small decrease in the production of elements up to magnesium and an increase in elements from chlorine to chromium. The effect on the mass fractions is much smaller than that owing to variations in the ${ }^{12} \mathrm{C}$ fusion rate.

We give in Table IV the sensitivity of the yield of each one of the species included in Table II to the rate of fusion reactions. There, $D_{i}$ is the logarithmic derivative of the mass ejected of species $i$ with respect to the enhancement factor of each fusion reaction, $f_{0}$ [note that when using Eq. (4), $f_{0}$ represents the maximum enhancement factor, attained at low

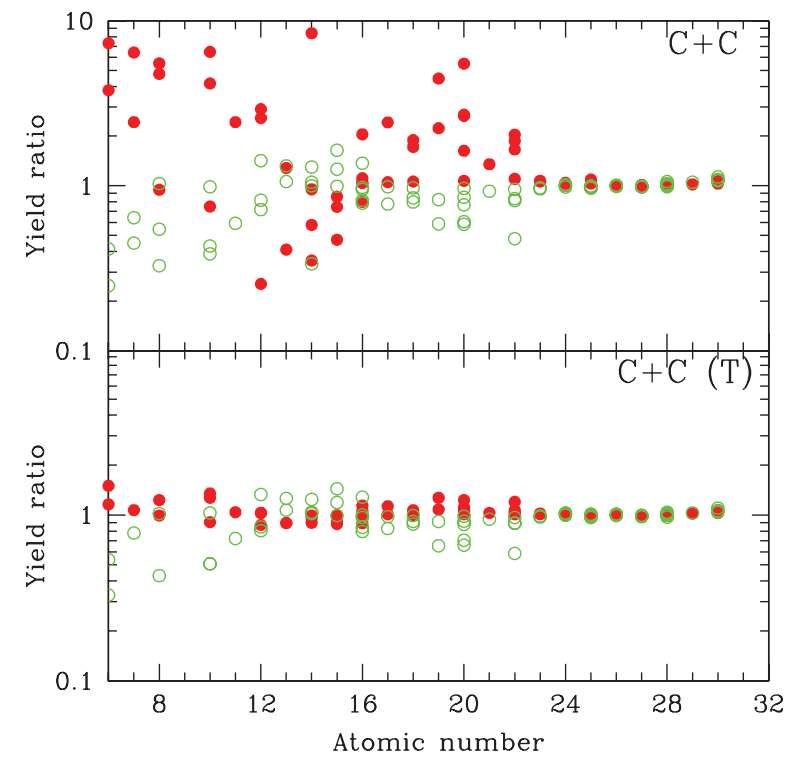

FIG. 7. (Color online) Same as Fig. 5, but plotted as a function of the atomic number of the product nucleus. Note that not all the isotopes shown here appear in Table IV. (Top) Rate multiplied by a fixed factor, either $\times 10$ (green open circles) or $\times 0.1$ (red solid circles). (Bottom) Rate multiplied by a factor function of temperature given by Eq. (4), with either $f_{0}=10$ (green open circles) or $f_{0}=0.1$ (red solid circles).

temperatures],

$$
D_{i}=\frac{\mathrm{d} \log _{10} m_{i}}{\mathrm{~d} \log _{10} f_{0}} \approx 0.5 \log _{10}\left(\frac{m_{i, 10}}{m_{i, 0.1}}\right),
$$

where $m_{i, 10}$ is the mass ejected of species $i$ for $f_{0}=10$ and $m_{i, 0.1}$ is the corresponding mass when $f_{0}=0.1$. According to this definition, a value of $D_{i} \approx 0.3$ means that the abundance of species $i$ approximately doubles for a constant enhancement factor of $f_{0}=10$ in the corresponding fusion reaction rate. Similarly, a relative change in the abundance of a species by $12 \%$ would correspond to $D_{i} \approx 0.05$, and a change by $2 \%$ would derive from $D_{i} \approx 0.01$.

Most notable is the robustness of the production of most Fegroup isotopes, notably of ${ }^{56} \mathrm{Ni}$. When the enhancement factor is computed from Eq. (4), there is no species with $\left|D_{i}\right|>0.1$, neither with respect to the rate of ${ }^{12} \mathrm{C}+{ }^{12} \mathrm{C}$ nor with respect to the ${ }^{16} \mathrm{O}+{ }^{16} \mathrm{O}$ rate, with the exceptions of ${ }^{12} \mathrm{C}$ and ${ }^{39} \mathrm{~K}$, respectively.

\section{B. Rate modified only in the nucleosynthetic code}

Figures 9 and 10 show the impact of the changes in the fusion rates of ${ }^{12} \mathrm{C}$ and of ${ }^{16} \mathrm{O}$ on the nucleosynthesis of type Ia supernovae when the rates are modified only in the nucleosynthetic code. They can be compared with Figs. 7 and 8, respectively, to evaluate the relevance of incorporating the modified rates into the hydrodynamic code. The trends visible in these figures are qualitatively similar, irrespective of whether the reaction rate has been modified in the hydrodynamic calculations. 


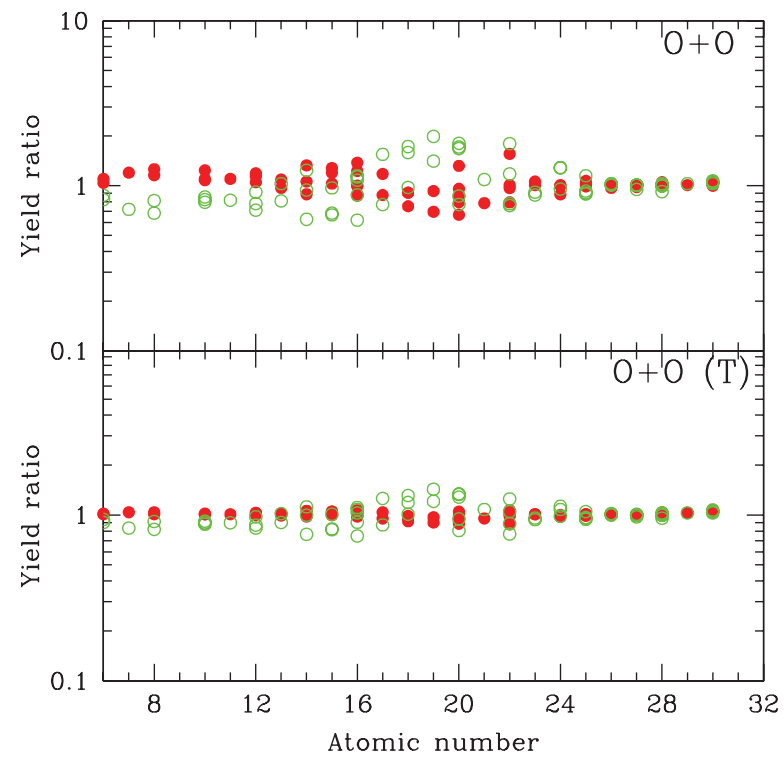

FIG. 8. (Color online) Same as Fig. 6, but plotted as a function of the atomic number of the product nucleus. Note that not all the isotopes shown here appear in Table IV. (Top) Rate multiplied by a fixed factor, either $\times 10$ (green open circles) or $\times 0.1$ (red solid circles). (Bottom) Rate multiplied by a factor function of temperature given by Eq. (4), with either $f_{0}=10$ (green open circles) or $f_{0}=0.1$ (red solid circles).

In Fig. 11 we show the yield ratios belonging to a modified rate of the $3 \alpha$ reaction. The influence of the rate of this reaction focuses on a few elements: nitrogen, nickel, copper, and zinc (specially the isotopes of nickel and zinc produced during $\alpha$-rich freeze-out of NSE) inversely correlate with the factor of enhancement of the $3 \alpha$ reaction, while titanium and, to a lesser extent, scandium, manganese, and iron (specially the isotopes produced during explosive Si burning) are more abundant when the $3 \alpha$ reaction is faster. These results can be explained by the fact that for a faster rate an $\alpha$-rich freeze-out occurs at lower temperature and density. As a result, the dotted line in Fig. 1 (left) shifts down when the $3 \alpha$ reaction is faster, increasing the yield of species made in normal freeze-out at the expense of $\alpha$-rich freeze-out products.

We give in Table $V$ the sensitivity of the yield of each one of the species included in Table II to the rate of fusion reactions, when they are modified only in the nucleosynthetic code. This table can be compared to Table IV to evaluate the importance of running a hydrodynamic code with the reaction rates modified or take the thermodynamic profiles of a reference model and modifying the rates only in a postprocessing code. One finds that the sensitivities shown in both tables are qualitatively similar. Although the precise values of $D_{i}$ for given species are not equal, the rating of the species that are most sensitive to any fusion reaction rate is the same in both tables. Given the pre-eminence of the fusion reaction rates with respect to the release of nuclear energy, we conclude that for SNI $a$ this kind of study can be safely carried out with a postprocessing code, using a set of thermodynamic trajectories obtained with a supernova hydrodynamics code where the reaction rates remain unchanged.

\section{SENSITIVITY TO THE RATE OF RADIATIVE CAPTURES AND TRANSFER REACTIONS}

We discuss in this section the sensitivity of the nucleosynthesis to changes in the rate of radiative captures and transfer reactions. We measure the sensitivity in a similar way as with respect to the rate of the fusion reactions, by defining $D_{i}$ as in Eq. (5). The meaning of $D_{i}$ is now the logarithmic derivative of the mass ejected of species $i$ with respect to the enhancement factor, $f_{0}$, of a reaction between particle $k$ and nucleus $j$, while $m_{i, 10}$ is the mass ejected of species $i$ when an enhancement factor $f_{0}=10$ (either fixed or function of temperature) is applied to the rate of the reactions $j+k \rightarrow l+m$ and $l+m \rightarrow j+k$, and $m_{i, 0.1}$ is the corresponding mass when $f_{0}=0.1$.

We start by analyzing the results obtained with a fixed enhancement factor (our first approach). In Sec. IV B we present the results obtained when we compute the enhancement factor with a decreasing uncertainty [see Eq. (4), our second approach]. Then, we select the reactions to which the nucleosynthesis is most sensitive and analyze in Sec. IV C the results achieved by adopting different prescriptions for their reaction rates, chosen among the most recent literature. Finally, in Sec. IV D we analyze the temperature ranges in which a modification of a given reaction rate affects most the chemical composition of the supernova ejecta.

\section{A. Fixed-rate enhancement factor}

Tables VI to XI give, for each reaction pair $j+k \rightleftarrows l+m$ that has a significant impact on the nucleosynthesis, the nuclei $i$ for which $\left|D_{i}\right|>0.3$ (more than twofold increase or decrease in the yield when the rate in enhanced or decreased by a factor of 10), and those for which $0.3>\left|D_{i}\right|>0.05$ (relative increase or decrease of the yield between $12 \%$ and a factor of 2). Although we only list in the tables the direct reactions, the inverse reactions contributed as well to the changes in the nucleosynthetic yield. There is only one reaction pair for which $\left|D_{i}\right|>1$; it is the ${ }^{30} \mathrm{Si}+\mathrm{p} \rightleftarrows{ }^{31} \mathrm{P}+\gamma$ reaction and the species whose abundance is mostly affected is ${ }^{35} \mathrm{~S}$. Each table shows the reactions belonging to a given type, for example $(n, \gamma)$, sorted according to the total mass they processed in our reference model, $\mathfrak{M}_{j k}$ (see Table III).

The species most sensitive to changes in the rates of $(n, \gamma)$ reactions (Table VI) are ${ }^{17} \mathrm{O},{ }^{26} \mathrm{Al},{ }^{21} \mathrm{Ne}$, and ${ }^{35} \mathrm{~S}$. The yields of all these nuclides are small, of order $10^{-7} M_{\odot}$. Apart from the neutron captures on iron isotopes, all the reactions listed in Table VI involve IMEs or CNO elements as the parent nuclides. Among the species with $\left|D_{i}\right|>0.05$, the most abundant are ${ }^{29} \mathrm{Si}$ and ${ }^{33} \mathrm{~S}$, both with yields on the order of a few times $10^{-4} M_{\odot}$, suggesting that the temperature range where $(n, \gamma)$ reaction rates most affect the final nucleosynthesis is approximately $2 \times 10^{9} \lesssim T \lesssim 4 \times 10^{9} \mathrm{~K}$, in agreement with our analysis in Sec. II B.

Radiative captures of protons are by far the group of reactions whose rate most strongly determines the final abundances of the supernova explosion, as can be deduced from Table VII. The reaction with the largest $\left|D_{i}\right|$ of the whole 
TABLE IV. Sensitivity of the nucleosynthesis to the rate of fusion reactions: Rate modified in the hydrodynamic and nucleosynthetic codes (see also Figs. 5 to 8 ). ${ }^{\text {a }}$

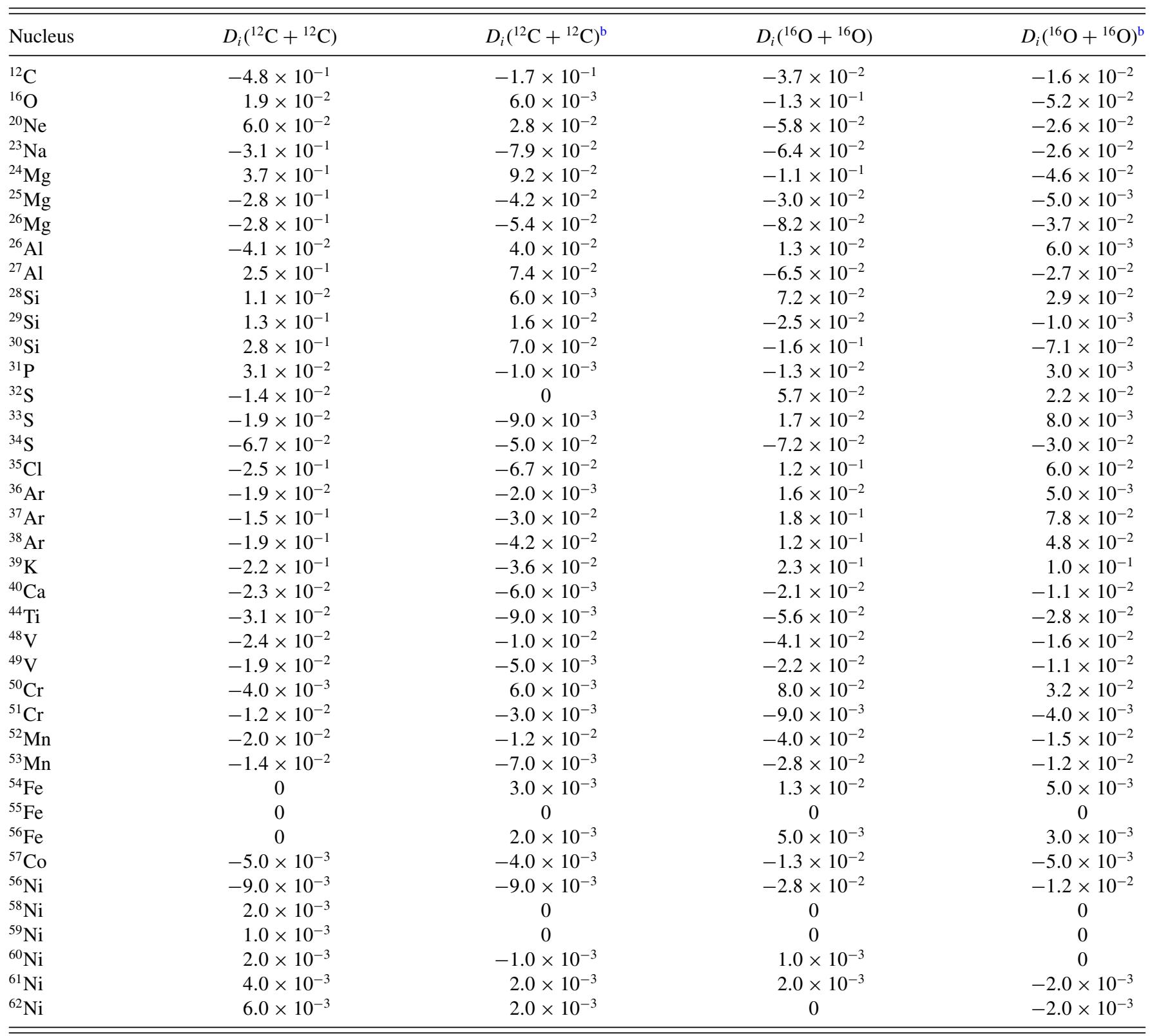

${ }^{\mathrm{a}}$ Values of $D_{i}$ less than $10^{-3}$ have been put to 0 .

${ }^{\mathrm{b}}$ Enhancement factor function of temperature according to Eq. (4).

network is ${ }^{30} \mathrm{Si}+p \rightleftarrows{ }^{31} \mathrm{P}+\gamma$, for which as many as 20 product species have $\left|D_{i}\right|>0.05$. The species most affected by changes in the rates of the proton capture group of reactions are ${ }^{14} \mathrm{~N},{ }^{26} \mathrm{Mg},{ }^{26} \mathrm{Al},{ }^{27} \mathrm{Al},{ }^{32} \mathrm{P},{ }^{35} \mathrm{~S}$, and ${ }^{43} \mathrm{Ca}$. Among these, ${ }^{27} \mathrm{Al}$ is the species with the largest yield, $m_{i}=4.6 \times 10^{-4} M_{\odot}$. Within the species with $0.3>\left|D_{i}\right|>0.05$ there are important products of the supernova explosion such as ${ }^{24} \mathrm{Mg},{ }^{25} \mathrm{Mg},{ }^{29} \mathrm{Si}$, ${ }^{30} \mathrm{Si},{ }^{31} \mathrm{P},{ }^{33} \mathrm{~S},{ }^{34} \mathrm{~S},{ }^{35} \mathrm{Cl},{ }^{38} \mathrm{Ar},{ }^{50} \mathrm{Cr},{ }^{51} \mathrm{Cr}$, and ${ }^{55} \mathrm{Fe}$. The parent nuclei involved in these reactions cover a wide range from ${ }^{14} \mathrm{C}$ to ${ }^{64} \mathrm{Ga}$.

The rates of $(p, n)$ reactions do not influence significantly the nucleosynthesis. The most affected species are ${ }^{32} \mathrm{P}$ and ${ }^{37} \mathrm{Cl}$, both with final yields on the order of $10^{-7} M_{\odot}$. Among the species with $\left|D_{i}\right|>0.05$, the most abundant are ${ }^{29} \mathrm{Si}$, with $m_{i}=4.5 \times 10^{-4} M_{\odot}$, and ${ }^{25} \mathrm{Mg}$ and ${ }^{49} \mathrm{~V}$, both with yields on the order of $10^{-5} M_{\odot}$.

There are several reactions of radiative capture of $\alpha$ particles that bear a non-negligible influence on the synthesis of large numbers of species (Table IX). The most notable is the reaction ${ }^{20} \mathrm{Ne}+\alpha \rightleftarrows{ }^{24} \mathrm{Mg}+\gamma$, for which there are 13 species with $\left|D_{i}\right|>0.3$ and 20 species with $0.3>\left|D_{i}\right|>0.05$, among them species with large abundances such as ${ }^{20} \mathrm{Ne},{ }^{24} \mathrm{Mg}$, ${ }^{28} \mathrm{Si},{ }^{36} \mathrm{Ar},{ }^{40} \mathrm{Ca}$, or ${ }^{52} \mathrm{Mn}$. Variations in the rate of $(\alpha, \gamma)$ reactions on ${ }^{24} \mathrm{Mg}$ and ${ }^{12} \mathrm{C}$ also influence the yields of large numbers of species, although not as much as does the reaction ${ }^{20} \mathrm{Ne}(\alpha, \gamma)$, while captures on ${ }^{28} \mathrm{Si}$ and ${ }^{32} \mathrm{~S}$ have a much 


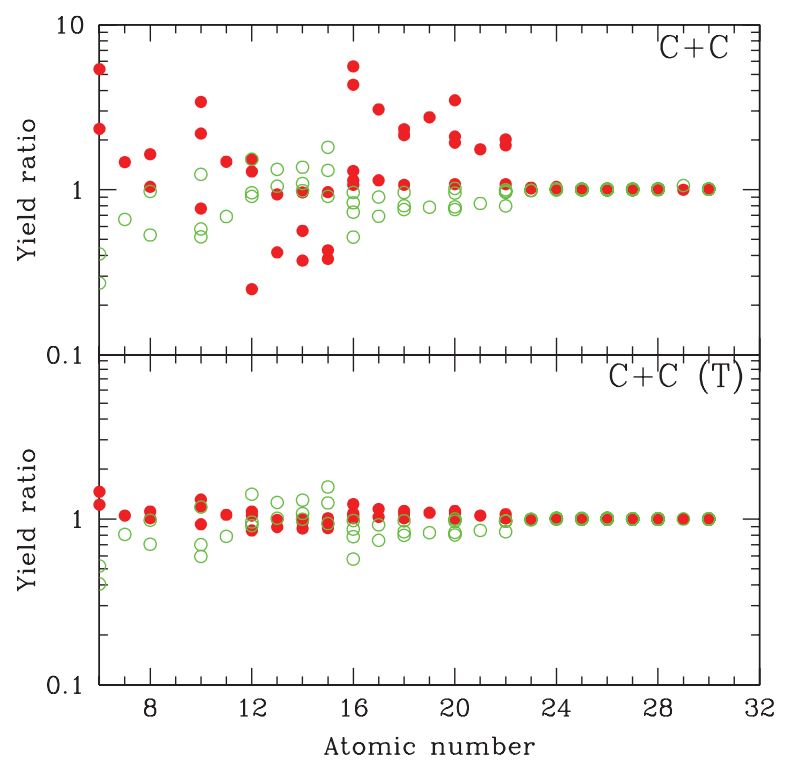

FIG. 9. (Color online) Ratio of mass ejected for each nuclide with a modified ${ }^{12} \mathrm{C}+{ }^{12} \mathrm{C}$ reaction rate with respect to the mass ejected in the reference model, as a function of the atomic number of the product nucleus. We modified the reaction rate only in the nucleosynthetic code. (Top) Rate multiplied by a fixed factor, either $\times 10$ (green open circles) or $\times 0.1$ (red solid circles). (Bottom) Rate multiplied by a factor function of temperature given by Eq. (4), with either $f_{0}=10$ (green open circles) or $f_{0}=0.1$ (red solid circles).

more limited reach. Note that, because we always modify coherently direct and inverse reaction rates to maintain detailed balance, the sensitivity to the reactions ${ }^{20} \mathrm{Ne}(\alpha, \gamma)^{24} \mathrm{Mg}$ and ${ }^{24} \mathrm{Mg}(\alpha, \gamma)^{28} \mathrm{Si}$ also pick up the effect of variations on ${ }^{24} \mathrm{Mg}(\gamma, \alpha){ }^{20} \mathrm{Ne}$ and ${ }^{28} \mathrm{Si}(\gamma, \alpha)^{24} \mathrm{Mg}$, respectively, which are reactions relevant for silicon burning. Most of the parent nuclei listed in the table belong to the IMEs group. An interesting exception is the reaction ${ }^{58} \mathrm{Ni}+\alpha \rightleftarrows{ }^{62} \mathrm{Zn}+\gamma$, which plays a relevant role in the $\alpha$-rich freeze-out of incinerated matter that leaves NSE at densities below $\sim 10^{8} \mathrm{~g} \mathrm{~cm}^{-3}$, because of the adiabatic expansion of the ejecta.

The species most affected by variations on the rate of $(\alpha, n)$ reactions, as well as the parent species listed in Table X, belong to the IMEs group. Among the species with $\left|D_{i}\right|>0.3$, the most abundant is ${ }^{26} \mathrm{Mg}$, whose yield is $2.7 \times 10^{-5} M_{\odot}$.

In Table XI, we give the sensitivities to the rates of $(\alpha, p)$ reactions. Abundant species most affected by variations of this type of reactions are ${ }^{23} \mathrm{Na},{ }^{26} \mathrm{Mg}$, and ${ }^{27} \mathrm{Al}$, the last with a yield of $4.6 \times 10^{-4} M_{\odot}$. As with $(p, \gamma)$ reactions, the parent species cover a wide range of baryon numbers, from ${ }^{13} \mathrm{~N}$ to ${ }^{62} \mathrm{Zn}$. The most influential reactions are ${ }^{13} \mathrm{~N}+\alpha \rightleftarrows{ }^{16} \mathrm{O}+p$, ${ }^{20} \mathrm{Ne}+\alpha \rightleftarrows{ }^{23} \mathrm{Na}+p,{ }^{23} \mathrm{Na}+\alpha \rightleftarrows{ }^{26} \mathrm{Mg}+p,{ }^{24} \mathrm{Mg}+\alpha \rightleftarrows$ ${ }^{27} \mathrm{Al}+p$, and ${ }^{27} \mathrm{Al}+\alpha \rightleftarrows{ }^{30} \mathrm{Si}+p$. There are present as well several reactions relevant for the $\alpha$-rich freeze-out of NSE, such as ${ }^{56} \mathrm{Ni}+\alpha \rightleftarrows{ }^{59} \mathrm{Cu}+p$ and ${ }^{58} \mathrm{Ni}+\alpha \rightleftarrows{ }^{61} \mathrm{Cu}+p$.

Several reactions appear in the tables that are responsible for bridging the gap between the QSE groups (the QSE groups of silicon and iron) in silicon burning [53], such as ${ }^{45} \mathrm{Sc}+p \rightleftarrows$ ${ }^{46} \mathrm{Ti}+\gamma,{ }^{42} \mathrm{Ca}+\alpha \rightleftarrows{ }^{46} \mathrm{Ti}+\gamma$, and ${ }^{44} \mathrm{Ti}+\alpha \rightleftarrows{ }^{47} \mathrm{~V}+p$. It is remarkable that the set of abundances significantly affected

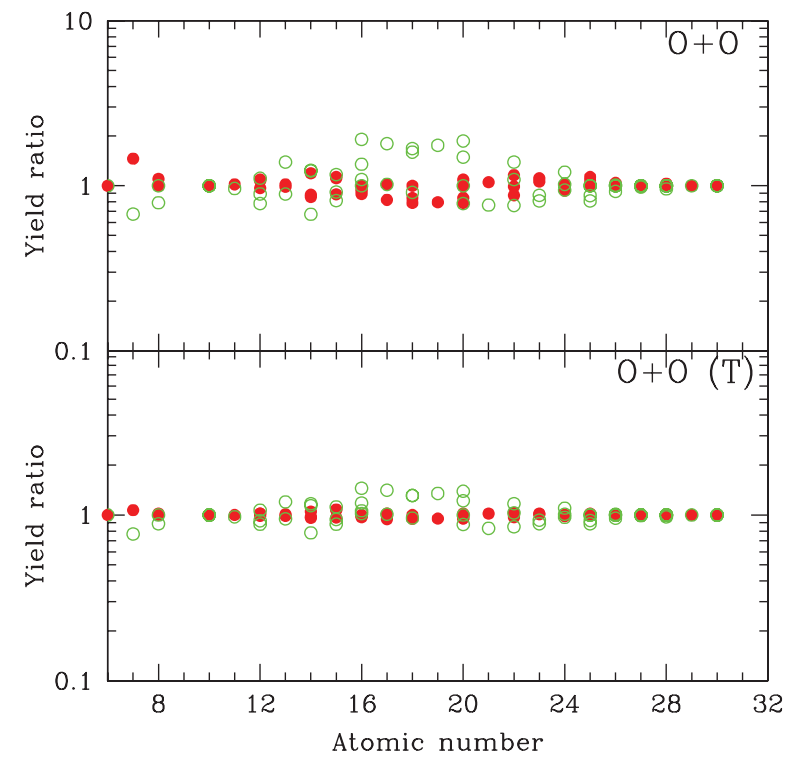

FIG. 10. (Color online) Ratio of mass ejected for each nuclide with a modified ${ }^{16} \mathrm{O}+{ }^{16} \mathrm{O}$ reaction rate with respect to the mass ejected in the reference model, as a function of the atomic number of the product nucleus. We modified the reaction rate only in the nucleosynthetic code. (Top) Rate multiplied by a fixed factor, either $\times 10$ (green open circles) or $\times 0.1$ (red solid circles). (Bottom) Rate multiplied by a factor function of temperature given by Eq. (4), with either $f_{0}=10$ (green open circles) or $f_{0}=0.1$ (red solid circles).

by the modification of the rates of these reactions is limited to species that fall into the QSE groups gap, but there appear very few species belonging to the QSE groups. When one of these

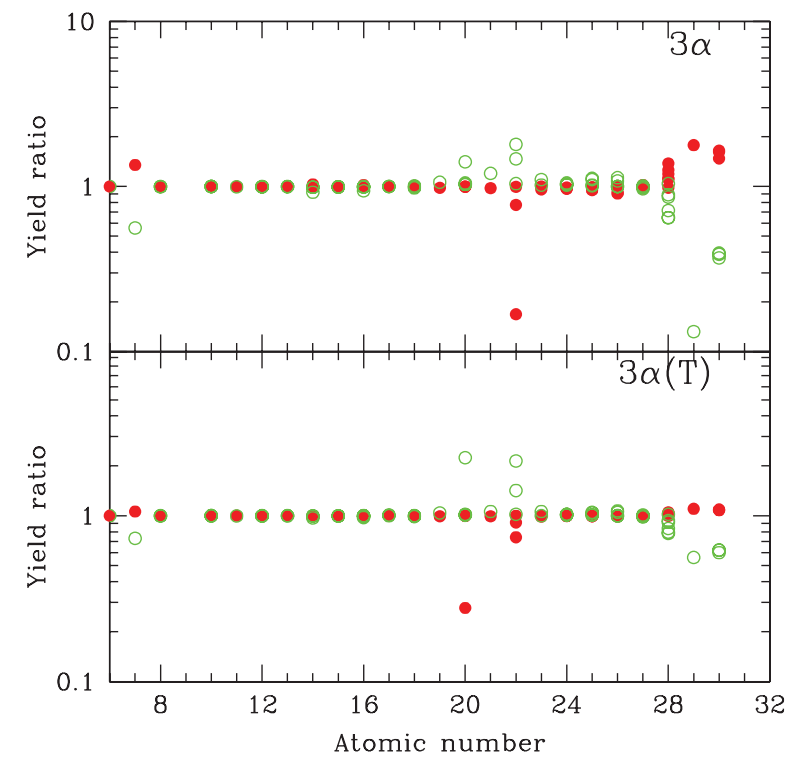

FIG. 11. (Color online) Ratio of mass ejected for each nuclide when the $3 \alpha$ reaction rate is modified to the mass ejected in the reference model, as a function of the atomic number of the product nucleus. We modified the reaction rate only in the nucleosynthetic code. (Top) Rate multiplied by a fixed factor, either $\times 10$ (green open circles) or $\times 0.1$ (red solid circles). (Bottom) Rate multiplied by a factor function of temperature given by Eq. (4), with either $f_{0}=10$ (green open circles) or $f_{0}=0.1$ (red solid circles). 
TABLE V. Sensitivity of the nucleosynthesis to the rate of fusion reactions: Rate modified only in the nucleosynthetic code (see also Figs. 9 to 11$){ }^{\text {a }}$

\begin{tabular}{|c|c|c|c|c|c|c|}
\hline Nucleus & $D_{i}\left({ }^{12} \mathrm{C}+{ }^{12} \mathrm{C}\right)$ & $D_{i}\left({ }^{12} \mathrm{C}+{ }^{12} \mathrm{C}\right)^{\mathrm{b}}$ & $D_{i}\left({ }^{16} \mathrm{O}+{ }^{16} \mathrm{O}\right)$ & $D_{i}\left({ }^{16} \mathrm{O}+{ }^{16} \mathrm{O}\right){ }^{\mathrm{b}}$ & $D_{i}(3 \alpha)$ & $D_{i}(3 \alpha)^{\mathrm{b}}$ \\
\hline${ }^{12} \mathrm{C}$ & $-3.8 \times 10^{-1}$ & $-1.9 \times 10^{-1}$ & 0 & 0 & 0 & 0 \\
\hline${ }^{16} \mathrm{O}$ & $-1.3 \times 10^{-2}$ & $-6.0 \times 10^{-3}$ & $-7.2 \times 10^{-2}$ & $-3.1 \times 10^{-2}$ & $-1.0 \times 10^{-3}$ & 0 \\
\hline${ }^{23} \mathrm{Na}$ & $-1.7 \times 10^{-1}$ & $-6.5 \times 10^{-2}$ & $-1.3 \times 10^{-2}$ & $-5.0 \times 10^{-3}$ & $-1.0 \times 10^{-3}$ & 0 \\
\hline${ }^{24} \mathrm{Mg}$ & $3.9 \times 10^{-1}$ & $1.1 \times 10^{-1}$ & $-6.8 \times 10^{-2}$ & $-3.2 \times 10^{-2}$ & $-1.0 \times 10^{-3}$ & 0 \\
\hline${ }^{25} \mathrm{Mg}$ & $-6.4 \times 10^{-2}$ & $-2.0 \times 10^{-2}$ & $3.0 \times 10^{-2}$ & $1.7 \times 10^{-2}$ & 0 & 0 \\
\hline${ }^{27} \mathrm{Al}$ & $2.5 \times 10^{-1}$ & $7.4 \times 10^{-2}$ & $-3.0 \times 10^{-2}$ & $-1.4 \times 10^{-2}$ & $-1.0 \times 10^{-3}$ & 0 \\
\hline${ }^{28} \mathrm{Si}$ & $5.0 \times 10^{-3}$ & 0 & $7.4 \times 10^{-2}$ & $3.4 \times 10^{-2}$ & $-2.4 \times 10^{-2}$ & $-7.0 \times 10^{-3}$ \\
\hline${ }^{29} \mathrm{Si}$ & $1.4 \times 10^{-1}$ & $2.7 \times 10^{-2}$ & $7.9 \times 10^{-2}$ & $4.3 \times 10^{-2}$ & $-2.0 \times 10^{-3}$ & $-1.0 \times 10^{-3}$ \\
\hline${ }^{30} \mathrm{Si}$ & $2.8 \times 10^{-1}$ & $8.5 \times 10^{-2}$ & $-1.2 \times 10^{-1}$ & $-6.4 \times 10^{-2}$ & $-2.0 \times 10^{-3}$ & $-1.0 \times 10^{-3}$ \\
\hline${ }^{31} \mathrm{P}$ & $-1.3 \times 10^{-2}$ & $-1.6 \times 10^{-2}$ & $6.0 \times 10^{-2}$ & $3.2 \times 10^{-2}$ & $-2.0 \times 10^{-3}$ & 0 \\
\hline${ }^{36} \mathrm{Ar}$ & $-2.3 \times 10^{-2}$ & $-7.0 \times 10^{-3}$ & $-2.0 \times 10^{-2}$ & $-9.0 \times 10^{-3}$ & $-7.0 \times 10^{-3}$ & $-2.0 \times 10^{-3}$ \\
\hline${ }^{37} \mathrm{Ar}$ & $-2.1 \times 10^{-1}$ & $-5.5 \times 10^{-2}$ & $1.7 \times 10^{-1}$ & $7.0 \times 10^{-2}$ & $2.0 \times 10^{-3}$ & 0 \\
\hline${ }^{38} \mathrm{Ar}$ & $-2.4 \times 10^{-1}$ & $-7.4 \times 10^{-2}$ & $1.4 \times 10^{-1}$ & $6.9 \times 10^{-2}$ & $-2.0 \times 10^{-3}$ & 0 \\
\hline${ }^{39} \mathrm{~K}$ & $-2.7 \times 10^{-1}$ & $-6.0 \times 10^{-2}$ & $1.7 \times 10^{-1}$ & $7.6 \times 10^{-2}$ & $1.7 \times 10^{-2}$ & $1.0 \times 10^{-2}$ \\
\hline${ }^{40} \mathrm{Ca}$ & $-2.6 \times 10^{-2}$ & $-9.0 \times 10^{-3}$ & $-7.2 \times 10^{-2}$ & $-3.3 \times 10^{-2}$ & $6.0 \times 10^{-3}$ & $2.0 \times 10^{-3}$ \\
\hline${ }^{44} \mathrm{Ti}$ & $-2.4 \times 10^{-2}$ & $-9.0 \times 10^{-3}$ & $-9.2 \times 10^{-2}$ & $-4.2 \times 10^{-2}$ & $1.4 \times 10^{-1}$ & $9.6 \times 10^{-2}$ \\
\hline${ }^{48} \mathrm{~V}$ & $-7.0 \times 10^{-3}$ & $-2.0 \times 10^{-3}$ & $-6.8 \times 10^{-2}$ & $-3.0 \times 10^{-2}$ & $2.9 \times 10^{-2}$ & $1.5 \times 10^{-2}$ \\
\hline${ }^{49} \mathrm{~V}$ & $-8.0 \times 10^{-3}$ & $-1.0 \times 10^{-3}$ & $-4.1 \times 10^{-2}$ & $-1.7 \times 10^{-2}$ & $5.0 \times 10^{-3}$ & 0 \\
\hline${ }^{50} \mathrm{Cr}$ & $-6.0 \times 10^{-3}$ & $3.0 \times 10^{-3}$ & $5.6 \times 10^{-2}$ & $2.4 \times 10^{-2}$ & $1.4 \times 10^{-2}$ & $5.0 \times 10^{-3}$ \\
\hline${ }^{51} \mathrm{Cr}$ & 0 & $3.0 \times 10^{-3}$ & $-1.8 \times 10^{-2}$ & $-7.0 \times 10^{-3}$ & $1.7 \times 10^{-2}$ & $5.0 \times 10^{-3}$ \\
\hline${ }^{52} \mathrm{Mn}$ & $2.0 \times 10^{-3}$ & $-1.0 \times 10^{-3}$ & $-7.3 \times 10^{-2}$ & $-3.1 \times 10^{-2}$ & $3.5 \times 10^{-2}$ & $1.3 \times 10^{-2}$ \\
\hline${ }^{60} \mathrm{Ni}$ & $2.0 \times 10^{-3}$ & 0 & 0 & 0 & $-1.1 \times 10^{-1}$ & $-4.5 \times 10^{-2}$ \\
\hline${ }^{61} \mathrm{Ni}$ & $2.0 \times 10^{-3}$ & 0 & 0 & 0 & $-1.4 \times 10^{-1}$ & $-5.8 \times 10^{-2}$ \\
\hline${ }^{62} \mathrm{Ni}$ & $2.0 \times 10^{-3}$ & 0 & 0 & 0 & $-1.6 \times 10^{-1}$ & $-6.4 \times 10^{-2}$ \\
\hline
\end{tabular}

${ }^{\mathrm{a}}$ Values of $D_{i}$ less than $10^{-3}$ have been put to 0 .

${ }^{b}$ Enhancement factor function of temperature according to Eq. (4).

reactions is modified, the increase or decrease it produces in the flux between QSE groups is offset against a slight adjustment in the abundances of other species located within the gap, therefore leaving the abundances of the species in QSE nearly unchanged. For instance, modifying the rate of ${ }^{42} \mathrm{Ca}+\alpha \rightleftarrows$ ${ }^{46} \mathrm{Ti}+\gamma$ by a factor of $\times 10$, the global flux carried by all the reactions that bridge the QSE gap changes by less than 5\%, in spite of an increase in the flux carried by the ${ }^{42} \mathrm{Ca}+\alpha \rightleftarrows$ ${ }^{46} \mathrm{Ti}+\gamma$ reaction by a factor of $\times 7$ (together with a decrease of the final yield of ${ }^{42} \mathrm{Ca}$ by a factor of $\times 0.7$ and an increase of the final yield of ${ }^{46} \mathrm{Ti}$ by a factor of $\times 1.8$ ). The larger flux carried by ${ }^{42} \mathrm{Ca}+\alpha \rightleftarrows{ }^{46} \mathrm{Ti}+\gamma$ is offset by a decrease in the fluxes owing to ${ }^{44} \mathrm{Ti}+\alpha \rightleftarrows{ }^{47} \mathrm{~V}+p$, and other reactions within the gap.
We have plotted in Fig. 12 the reactions with the largest $\max \left(\left|D_{i}\right|\right)$ in Tables VI to XI. It is remarkable that no reaction appears involving the main products of SNIa, that is, elements from the Fe-group nuclei, except ${ }^{47} \mathrm{Cr}+\alpha \rightleftarrows{ }^{50} \mathrm{Mn}+p$. This reaction might play a role in the freeze-out from incomplete silicon burning [54], as one of the last links between the two main QSE groups. The rest of the reactions in the plot sketch a connected network from ${ }^{12} \mathrm{C}$ up to ${ }^{37} \mathrm{Ar}$. The pattern displayed in the figure suggests that we can talk not only of the reactions whose rates are most influential in the supernova yields, but also of a path in the nuclear chart that is most influential. The main stream involves $(\alpha, \gamma)$ reactions from ${ }^{12} \mathrm{C}$ to ${ }^{28} \mathrm{Si}$, although the step from ${ }^{16} \mathrm{O}$ to ${ }^{20} \mathrm{Ne}$ is not covered by the ${ }^{16} \mathrm{O}+\alpha \rightleftarrows{ }^{20} \mathrm{Ne}+\gamma$ reaction pair but by the combination 
TABLE VI. Sensitivity of the nucleosynthesis to the rate of $(n, \gamma)$ reactions with the parent nuclide given in the first column. ${ }^{a}$

\begin{tabular}{|c|c|c|c|}
\hline${ }^{28} \mathrm{Si}$ & 0.30 & ${ }^{35} \mathrm{~S}$ & ${ }^{21} \mathrm{Ne},{ }^{25} \mathrm{Mg},{ }^{26} \mathrm{Al},{ }^{33} \mathrm{~S}$ \\
\hline${ }^{55} \mathrm{Fe}$ & 0.20 & & ${ }^{54,55} \mathrm{Mn},{ }^{57,58} \mathrm{Fe},{ }^{58,59} \mathrm{Co}$ \\
\hline${ }^{36} \mathrm{Ar}$ & 0.093 & & ${ }^{37} \mathrm{Cl},{ }^{37} \mathrm{Ar}$ \\
\hline${ }^{44} \mathrm{Ti}$ & 0.076 & & ${ }^{45} \mathrm{Sc}$ \\
\hline${ }^{24} \mathrm{Mg}$ & 0.045 & ${ }^{26} \mathrm{Al},{ }^{35} \mathrm{~S}$ & ${ }^{17} \mathrm{O},{ }^{21} \mathrm{Ne},{ }^{25} \mathrm{Mg},{ }^{32} \mathrm{P},{ }^{33} \mathrm{~S}$ \\
\hline${ }^{56} \mathrm{Fe}$ & 0.0093 & & ${ }^{57} \mathrm{Fe}$ \\
\hline${ }^{16} \mathrm{O}$ & 0.0092 & ${ }^{17} \mathrm{O}$ & ${ }^{25} \mathrm{Mg}$ \\
\hline${ }^{46} \mathrm{Ti}$ & 0.0068 & & ${ }^{46} \mathrm{Ti}$ \\
\hline${ }^{29} \mathrm{Si}$ & 0.0061 & & ${ }^{29} \mathrm{Si}$ \\
\hline${ }^{20} \mathrm{Ne}$ & 0.0049 & ${ }^{21} \mathrm{Ne}$ & \\
\hline${ }^{33} \mathrm{~S}$ & 0.0033 & & ${ }^{33} \mathrm{P}$ \\
\hline
\end{tabular}

a The reactions listed are those that processed more than $10^{-6} M_{\odot}$ in the reference model (see Table III) and with any max $\left(\left|D_{i}\right|\right)>0.05$.

of ${ }^{16} \mathrm{O}+n \rightleftarrows{ }^{17} \mathrm{O}+\gamma$ and ${ }^{17} \mathrm{O}+\alpha \rightleftarrows{ }^{20} \mathrm{Ne}+n$. Above $\mathrm{Mg}$, there appear many branches owing to a number of $(\alpha, p)$ and $(\alpha, n)$ reactions and their inverses, which shift the stream to the side of moderately neutron-rich nuclei. The path ends in a loop involving the reactions ${ }^{34} \mathrm{~S}+\alpha \rightleftarrows{ }^{37} \mathrm{Ar}+n,{ }^{37} \mathrm{Ar}+n \rightleftarrows$ ${ }^{37} \mathrm{Cl}+p$, and ${ }^{37} \mathrm{Cl}+p \rightleftarrows{ }^{34} \mathrm{~S}+\alpha$.

The right panel of Fig. 12 shows the path defined by the reactions that carry the largest mass flow during the SNIa explosion, computed through Eq. (3), together with the path of most influential reactions. A couple of points have to be retained to make a meaningful comparison between both paths. First, as explained in Sec. IIC, in shells achieving NSE, the mass flow prior to equilibrium has not been accounted for. Hence, Fig. 12 does not reflect the nuclear flow from carbon and oxygen up to the Fe group in incinerated shells, giving the impression that the Si group and the Fe group are disconnected. Second, our consideration of a nuclear reaction as highly influential is based on the relative variation of the yield of any species that has $\max \left(\left|D_{i}\right|\right)>0.3$. Thus, it is possible for a nuclear reaction off the maximum mass flow path to affect significatively the abundance of trace species. The maximum mass flow path follows the $Z=N$ line from carbon to the $\mathrm{Si}$ group, but deviates to the neutron-rich side within the $\mathrm{Fe}$ group, which accumulates the neutron excess owing to the initial metallicity and the electron captures close to the center of the star (see Fig. 1). Consequently, and owing to the small abundance of neutrons, the connection between both groups is provided mainly by $(\alpha, p)$ reactions. It is evident from Fig. 12 that the most influential path occupies a region slightly more neutron rich as compared to the maximum mass flow path, especially below $A \sim 28$. The species most affected by reactions that lay off the maximum mass flow path are trace species, for example, ${ }^{17} \mathrm{O},{ }^{21} \mathrm{Ne}$, ${ }^{25} \mathrm{Mg},{ }^{37} \mathrm{Cl}$, and ${ }^{47} \mathrm{Ti}$. Reactions within the Si group form a dense network in which the rate of a particular reaction loses relevance, and the same applies to reactions within the Fe group.
Tables XII and XIII give, for the most important product nuclei, $i$, the reactions $j+k \rightleftarrows l+m$ that have the largest impact on its yield, $\left|D_{i}\right|$. We have included in these tables only the species with mass fraction greater than $10^{-5}$, or that are interesting radioactive isotopes, and with $\max \left(\left|D_{i}\right|\right)>0.01$. We show as well the production factor of these species (in our reference model), to help in evaluating the relevance of the modifications to the yield of each nuclide. For radioactive isotopes, we have calculated the production factor taking as a reference the solar abundance of the end product of the disintegration chain. For each nucleus, we show a maximum of ten reactions, sorted by $\left|D_{i}\right|$.

Among the species with the largest production factors, the yields of ${ }^{28} \mathrm{Si}$ and ${ }^{32} \mathrm{~S}$ are hardly affected by any rate (Table XII), the maximum $\left|D_{i}\right|$ being 0.09 and 0.04 , respectively (both owing to the reaction ${ }^{13} \mathrm{~N}+\alpha \rightleftarrows{ }^{16} \mathrm{O}+p$ ), implying relative variations on their yields of $23 \%$ and $10 \%$ when the rates change by a factor of 10 . The same applies to ${ }^{36} \mathrm{Ar}$, whose maximum $\left|D_{i}\right|$ is 0.09 (Table XIII), while the yield of ${ }^{40} \mathrm{Ca}$ is slightly more dependent on the rates of the radiative $\alpha$ captures on ${ }^{20} \mathrm{Ne}$ and ${ }^{12} \mathrm{C}$ and on the $(\alpha, p)$ reaction on ${ }^{13} \mathrm{~N}$, with $\left|D_{i}\right|$ up to 0.15 (variation of up to $40 \%$ of the yield for a rate change by a factor of 10 ). Isotopes belonging to the $\mathrm{Fe}$ group with production factor larger than 100 have a similar level of sensitivity to the variation of the reaction rates with maximum $\left|D_{i}\right|$ slightly above $\sim 0.1$, with the exception of ${ }^{56} \mathrm{Ni},{ }^{58} \mathrm{Ni},{ }^{54} \mathrm{Fe}$, and ${ }^{56} \mathrm{Fe}$ (the last two do not appear in the table), whose yields are quite robust.

The list of reactions that are most influential on ${ }^{44} \mathrm{Ti}$ synthesis has some points in common with that found in Ref. [19] in the context of core-collapse supernovae as, for instance, ${ }^{44} \mathrm{Ti}+\alpha \rightleftarrows{ }^{47} \mathrm{~V}+p,{ }^{40} \mathrm{Ca}+\alpha \rightleftarrows{ }^{44} \mathrm{Ti}+\gamma,{ }^{12} \mathrm{C}+$ $\alpha \rightleftarrows{ }^{16} \mathrm{O}+\gamma$, and the $3 \alpha$ reaction. However, in SNIa there are no reactions involving nuclei heavier than Ti that affect significantly the yield of ${ }^{44} \mathrm{Ti}$, at variance with what was found 
TABLE VII. Sensitivity of the nucleosynthesis to the rate of $(p, \gamma)$ reactions with parent nuclide given in the first column. ${ }^{\text {a }}$





in Ref. [19] for core-collapse supernovae. The main reason is that, in our models, ${ }^{44} \mathrm{Ti}$ is made in moderately neutronized matter $(\eta \gtrsim 0.001)$. In QSE, while the composition of the Si group is nearly independent of the neutron excess, that of the Fe group is strongly affected [53]. Thus, an increase in the neutron excess favors the equilibrium linking of ${ }^{44} \mathrm{Ti}$ (a $\eta=0$ nucleus) to the $\mathrm{Si}$ group, leading to a low sensitivity of its abundance to the rate of the reactions within the $\mathrm{Fe}$ group. Such a progressive decrease of the importance of the Fe-group reactions for the production of ${ }^{44} \mathrm{Ti}$ as $\eta$ increases can also be deduced from comparison of Tables 4, 7, and 8 in Ref. [19].

\section{B. Enhancement factor function of temperature}

Tables XIV to XIX are the same as Tables VI to XI, except that the enhancement factor $f$ is a function of temperature, given by Eq. (4).

A general result that applies to all the rates shown in Tables XIV to XIX is that the sensitivities drop (in absolute value) strongly as compared to the case of fixed enhancement factor. Very few reactions have $\left|D_{i}\right|>0.3$ when we compute the enhancement factor using Eq. (4). The list of species sensitive to the rates of $(n, \gamma)$ reactions is much shorter, and the only product species with $\left|D_{i}\right|>0.3$ in this list is ${ }^{21} \mathrm{Ne}$. 
TABLE VIII. Sensitivity of the nucleosynthesis to the rate of $(p, n)$ reactions with parent nuclide given in the first column. ${ }^{\mathrm{a}}$

\begin{tabular}{|c|c|c|c|}
\hline${ }^{53} \mathrm{Mn}$ & 0.61 & \multirow{6}{*}{${ }^{32} \mathrm{P}$} & ${ }^{53} \mathrm{Mn}$ \\
\hline${ }^{57} \mathrm{Co}$ & 0.57 & & ${ }^{57} \mathrm{Co},{ }^{61} \mathrm{Ni},{ }^{65} \mathrm{Zn}$ \\
\hline${ }^{32} \mathrm{P}$ & 0.44 & & \\
\hline${ }^{56} \mathrm{Fe}$ & 0.31 & & ${ }^{55} \mathrm{Mn},{ }^{57,58} \mathrm{Fe}$ \\
\hline${ }^{58} \mathrm{Co}$ & 0.27 & & ${ }^{58} \mathrm{Co}$ \\
\hline${ }^{47} \mathrm{Ti}$ & 0.12 & & ${ }^{46} \mathrm{Ti}$ \\
\hline${ }^{27} \mathrm{Al}$ & 0.12 & \multirow{6}{*}{${ }^{37} \mathrm{Cl}$} & ${ }^{26} \mathrm{Al}$ \\
\hline${ }^{54} \mathrm{Mn}$ & 0.11 & & ${ }^{54} \mathrm{Mn}$ \\
\hline${ }^{44} \mathrm{Sc}$ & 0.072 & & ${ }^{47} \mathrm{Ti}$ \\
\hline${ }^{55} \mathrm{Mn}$ & 0.068 & & ${ }^{55} \mathrm{Mn}$ \\
\hline${ }^{45} \mathrm{Sc}$ & 0.064 & & ${ }^{45} \mathrm{Sc}$ \\
\hline${ }^{35} \mathrm{~S}$ & $3.4 \times 10^{-5}$ & & ${ }^{35} \mathrm{~S}$ \\
\hline
\end{tabular}



Radiative captures of protons suffer as well from a reduction of their influence on the supernova yields: ${ }^{30} \mathrm{Si}+p \rightleftarrows{ }^{31} \mathrm{P}+\gamma$ continues being the most influential reaction, but the number and importance of the species affected by its rate is much lower than with a fixed rate enhancement factor. The only species with $\left|D_{i}\right|>0.3$ are ${ }^{26} \mathrm{Al}$ and ${ }^{35} \mathrm{~S}$. The influence of $(p, n)$ reactions on the supernova yields is marginal when Eq. (4) is used to determine the enhancement factor of the rates, and the same applies to $(\alpha, p)$ reactions. Among the $(\alpha, \gamma)$ reactions, the capture on ${ }^{20} \mathrm{Ne}$ continues being the reaction with the largest list of product species with $\left|D_{i}\right|>0.05$. The only species with $\left|D_{i}\right|>0.3$ owing to variations on the rate of this type of reaction is the trace species ${ }^{43} \mathrm{Ca}$. Finally, the only species with $\left|D_{i}\right|>0.3$ with respect to variations of $(\alpha, n)$ reaction rates in Table XVIII are ${ }^{21} \mathrm{Ne}$ and ${ }^{43} \mathrm{Ca}$.

TABLE IX. Sensitivity of the nucleosynthesis to the rate of $(\alpha, \gamma)$ reactions with parent nuclide given in the first column. ${ }^{a}$

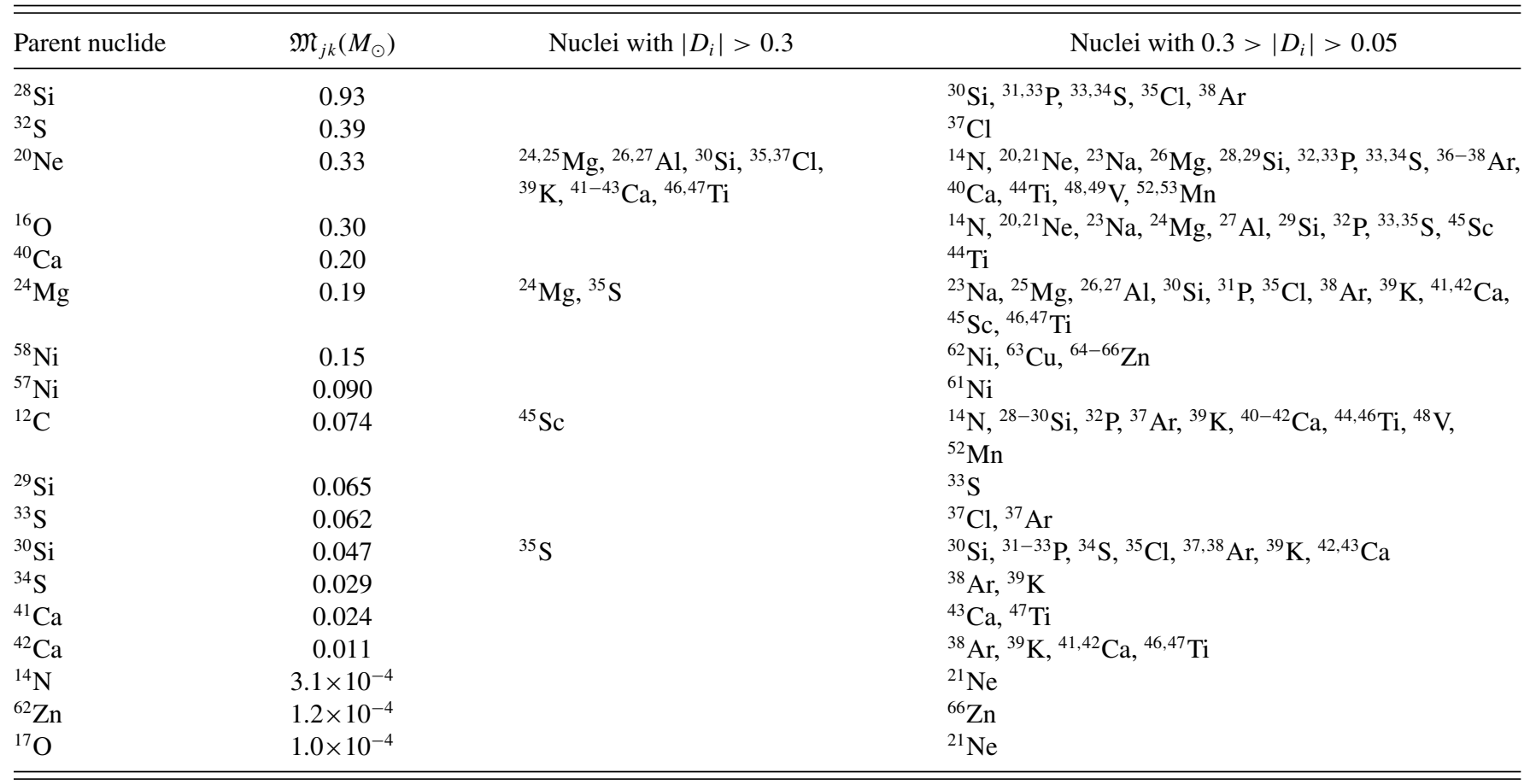

${ }^{\mathrm{a}}$ The reactions listed are those that processed more than $10^{-6} M_{\odot}$ in the reference model (see Table III) and with any max $\left(\left|D_{i}\right|\right)>0.05$. 
TABLE X. Sensitivity of the nucleosynthesis to the rate of $(\alpha, n)$ reactions with the parent nuclide given in the first column. ${ }^{\text {a }}$

\begin{tabular}{|c|c|c|c|}
\hline${ }^{29} \mathrm{Si}$ & 0.70 & ${ }^{35} \mathrm{~S}$ & ${ }^{21} \mathrm{Ne},{ }^{25} \mathrm{Mg},{ }^{26} \mathrm{Al},{ }^{29} \mathrm{Si},{ }^{34} \mathrm{~S},{ }^{35} \mathrm{Cl},{ }^{43} \mathrm{Ca},{ }^{47} \mathrm{Ti}$ \\
\hline${ }^{33} \mathrm{~S}$ & 0.64 & & ${ }^{37} \mathrm{Cl},{ }^{43} \mathrm{Ca}$ \\
\hline${ }^{30} \mathrm{Si}$ & 0.43 & ${ }^{33} \mathrm{P},{ }^{43} \mathrm{Ca}$ & ${ }^{29} \mathrm{Si},{ }^{32} \mathrm{P},{ }^{33,35} \mathrm{~S},{ }^{37} \mathrm{Cl},{ }^{47} \mathrm{Ti}$ \\
\hline${ }^{25} \mathrm{Mg}$ & 0.28 & ${ }^{26} \mathrm{Al}$ & ${ }^{17} \mathrm{O},{ }^{21} \mathrm{Ne},{ }^{25} \mathrm{Mg},{ }^{32} \mathrm{P},{ }^{35} \mathrm{~S}$ \\
\hline${ }^{34} \mathrm{~S}$ & 0.10 & ${ }^{37} \mathrm{Cl}$ & ${ }^{37} \mathrm{Ar}$ \\
\hline${ }^{46} \mathrm{Ti}$ & 0.058 & & ${ }^{46} \mathrm{Ti}$ \\
\hline${ }^{41} \mathrm{Ca}$ & 0.055 & & ${ }^{43} \mathrm{Ca},{ }^{47} \mathrm{Ti}$ \\
\hline${ }^{38} \mathrm{Ar}$ & 0.042 & & ${ }^{41} \mathrm{Ca}$ \\
\hline${ }^{21} \mathrm{Ne}$ & 0.014 & ${ }^{21} \mathrm{Ne}$ & \\
\hline${ }^{22} \mathrm{Ne}$ & 0.013 & & ${ }^{17} \mathrm{O},{ }^{22} \mathrm{Ne}$ \\
\hline${ }^{17} \mathrm{O}$ & 0.011 & ${ }^{17} \mathrm{O}$ & ${ }^{21} \mathrm{Ne}$ \\
\hline
\end{tabular}

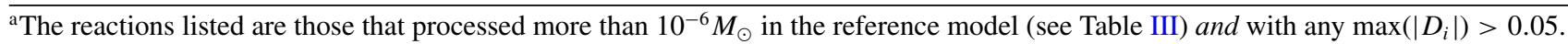

The rate enhancement factor computed with Eq. (4) differs most from the fixed enhancement factor at temperatures $T \gtrsim$ $3-5 \times 10^{9} \mathrm{~K}$. Thus, it has a stronger effect on reactions whose main role is played at high temperatures, as highlighted by comparing, once more, the list of reactions (parent nuclei) in Tables XIV to XIX with that in Tables VI to XI. For instance, as most $(n, \gamma)$ reactions influence the yields at temperatures of order $2 \times 10^{9} \lesssim T \lesssim 4 \times 10^{9} \mathrm{~K}$, the list of reactions in Tables VI and XIV is quite similar. However, the list of $(p, \gamma)$ reactions in Table $\mathrm{XV}$ is much shorter than in Table VII because the reduction of the enhancement factor at high temperatures affects most reactions with $\mathrm{Fe}$-group nuclei, while reactions with IMEs are less affected.

Tables XX and XXI are similar to Tables XII and XIII, except that the enhancement factor $f$ is now a function of temperature, given by Eq. (4). The maximum $\left|D_{i}\right|$ achieved with Eq. (4) for a given species is in general a factor of 2 smaller than when using a fixed rate enhancement factor, while all the species with a production factor larger than 100 have maximum $\left|D_{i}\right|<0.1$.

\section{Sensitivity to different rate prescriptions}

In this section, we analyze the changes in the yields obtained using different prescriptions for the rates of a few selected reactions. To this end, we have accessed the JINA REACLIB Database to compare the most recent rates for each one of the selected reactions. We discuss in the following the prescriptions for the reactions that appear in Tables VI to XI with maximum $\left|D_{i}\right|>0.3$. The results are presented in Table XXII in the form of percent variations of the yield of product species when two different prescriptions are used for each reaction rate. We give in the table as well the sources of the rates of each reaction. The reference rate (i.e., that used in the denominator of the calculation of the relative variation of the yield) is always that cited in second place in the table. All the references that appear in this section are taken from the JINA web page.

\section{1. $(n, \gamma)$ reactions}

The rates of the reactions we consider are fits to either theoretical or experimental results published in Refs. [42,5557].

The discrepancy between the different rates of the reactions ${ }^{32} \mathrm{~S}+n \rightleftarrows{ }^{33} \mathrm{~S}+\gamma,{ }^{28} \mathrm{Si}+n \rightleftarrows{ }^{29} \mathrm{Si}+\gamma,{ }^{24} \mathrm{Mg}+n \rightleftarrows$ ${ }^{25} \mathrm{Mg}+\gamma,{ }^{25} \mathrm{Mg}+n \rightleftarrows{ }^{26} \mathrm{Mg}+\gamma$, and ${ }^{20} \mathrm{Ne}+n \rightleftarrows{ }^{21} \mathrm{Ne}+$ $\gamma$ computed from the above references is less than a factor of 10 for $T \gtrsim 10^{9} \mathrm{~K}$. As this uncertainty is within the range explored in Sec. IV A, we do not deem it necessary to further analyze these reaction rates.

However, the rate of the reaction ${ }^{16} \mathrm{O}+n \rightleftarrows{ }^{17} \mathrm{O}+\gamma$ computed from the two references in JINA (Refs. [55] and [57]) shows a discrepancy of more than two orders of magnitude between these two cases. We have computed the nucleosynthesis of our SNIa model with both rates and compared the results in the first row of Table XXII. Aside from the trace product ${ }^{17} \mathrm{O}$, whose yield decreases by two orders of magnitude when using the rate from Ref. [57], the effect on each abundance is smaller than $27 \%$. We conclude that $(n, \gamma)$ reaction rates, in general, are not critical for obtaining accurate yields from SNIa models.

\section{2. $(p, \gamma)$ reactions}

The rates of the reactions we consider are fits to either theoretical or experimental results published in Refs. [42,56, 58,59,61].

The discrepancy between the different rates of the reactions ${ }^{25} \mathrm{Mg}+p \rightleftarrows{ }^{26} \mathrm{Al}+\gamma,{ }^{26} \mathrm{Mg}+p \rightleftarrows{ }^{27} \mathrm{Al}+\gamma,{ }^{26} \mathrm{Al}+$ $p \rightleftarrows{ }^{27} \mathrm{Si}+\gamma,{ }^{27} \mathrm{Al}+p \rightleftarrows{ }^{28} \mathrm{Si}+\gamma$, and ${ }^{30} \mathrm{Si}+p \rightleftarrows{ }^{31} \mathrm{P}+$ $\gamma$ computed from the above references is less than a factor of 10 for $T \gtrsim 10^{9} \mathrm{~K}$, well within the range explored in Sec. IV A. The reaction ${ }^{45} \mathrm{Sc}+p \rightleftarrows{ }^{46} \mathrm{Ti}+\gamma$, which contributes to the linking of QSE groups in silicon burning, is only evaluated in JINA through three somewhat different theoretical models. 
TABLE XI. Sensitivity of the nucleosynthesis to the rate of $(\alpha, p)$ reactions with parent nuclide given in the first column. ${ }^{\text {a }}$

\begin{tabular}{|c|c|c|c|}
\hline${ }^{28} \mathrm{Si}$ & 0.77 & & ${ }^{26} \mathrm{Mg},{ }^{31-33} \mathrm{P},{ }^{35} \mathrm{~S},{ }^{37} \mathrm{Cl}$ \\
\hline${ }^{30} \mathrm{P}$ & 0.68 & & ${ }^{43} \mathrm{Ca}$ \\
\hline${ }^{27} \mathrm{Al}$ & 0.63 & ${ }^{27} \mathrm{Al},{ }^{35} \mathrm{~S}$ & ${ }^{24-26} \mathrm{Mg},{ }^{26} \mathrm{Al},{ }^{29,30} \mathrm{Si},{ }^{32,33} \mathrm{P},{ }^{33} \mathrm{~S},{ }^{37} \mathrm{Cl},{ }^{43} \mathrm{Ca},{ }^{47} \mathrm{Ti}$ \\
\hline${ }^{31} \mathrm{P}$ & 0.61 & & ${ }^{30} \mathrm{Si},{ }^{31-33} \mathrm{P},{ }^{34,35} \mathrm{~S},{ }^{35} \mathrm{Cl}$ \\
\hline${ }^{24} \mathrm{Mg}$ & 0.58 & ${ }^{35} \mathrm{~S},{ }^{43} \mathrm{Ca}$ & ${ }^{24-26} \mathrm{Mg},{ }^{26,27} \mathrm{Al},{ }^{30} \mathrm{Si},{ }^{32} \mathrm{P},{ }^{34} \mathrm{~S},{ }^{35,37} \mathrm{Cl},{ }^{47} \mathrm{Ti}$ \\
\hline${ }^{33} \mathrm{~S}$ & 0.55 & & ${ }^{43} \mathrm{Ca}$ \\
\hline${ }^{39} \mathrm{~K}$ & 0.48 & & ${ }^{43} \mathrm{Ca},{ }^{47} \mathrm{Ti}$ \\
\hline${ }^{40} \mathrm{Ca}$ & 0.44 & & ${ }^{43} \mathrm{Ca}$ \\
\hline${ }^{29} \mathrm{Si}$ & 0.44 & & ${ }^{32} \mathrm{P}$ \\
\hline${ }^{13} \mathrm{~N}$ & 0.39 & & ${ }^{14} \mathrm{~N},{ }^{28} \mathrm{Si},{ }^{37,38} \mathrm{Ar},{ }^{40,43} \mathrm{Ca},{ }^{45} \mathrm{Sc},{ }^{44} \mathrm{Ti},{ }^{48,49} \mathrm{~V},{ }^{50} \mathrm{Cr},{ }^{52,53} \mathrm{Mn}$ \\
\hline${ }^{35} \mathrm{Cl}$ & 0.38 & & ${ }^{38} \mathrm{Ar},{ }^{39} \mathrm{~K},{ }^{41,42} \mathrm{Ca},{ }^{47} \mathrm{Ti}$ \\
\hline${ }^{57} \mathrm{Ni}$ & 0.17 & & ${ }^{61} \mathrm{Ni},{ }^{65} \mathrm{Zn}$ \\
\hline${ }^{48} \mathrm{Cr}$ & 0.13 & & ${ }^{48} \mathrm{~V},{ }^{49} \mathrm{~V}$ \\
\hline${ }^{45} \mathrm{Ti}$ & 0.12 & & ${ }^{45} \mathrm{Sc}$ \\
\hline${ }^{23} \mathrm{Na}$ & 0.12 & ${ }^{26} \mathrm{Mg},{ }^{43} \mathrm{Ca}$ & ${ }^{14} \mathrm{~N},{ }^{21} \mathrm{Ne},{ }^{23} \mathrm{Na},{ }^{29} \mathrm{Si},{ }^{32} \mathrm{P},{ }^{33} \mathrm{~S},{ }^{37} \mathrm{Cl},{ }^{40} \mathrm{Ca},{ }^{45} \mathrm{Sc},{ }^{44,47} \mathrm{Ti}$ \\
\hline${ }^{41} \mathrm{Ca}$ & 0.068 & & ${ }^{43} \mathrm{Ca},{ }^{47} \mathrm{Ti}$ \\
\hline${ }^{46} \mathrm{Ti}$ & 0.068 & & ${ }^{46} \mathrm{Ti}$ \\
\hline${ }^{35} \mathrm{Ar}$ & 0.061 & & ${ }^{43} \mathrm{Ca}$ \\
\hline${ }^{34} \mathrm{~S}$ & 0.054 & ${ }^{37} \mathrm{Cl}$ & \\
\hline${ }^{39} \mathrm{Ca}$ & 0.049 & & ${ }^{47} \mathrm{Ti}$ \\
\hline${ }^{30} \mathrm{Si}$ & 0.032 & & ${ }^{33} \mathrm{P}$ \\
\hline${ }^{42} \mathrm{Ca}$ & 0.020 & & ${ }^{46} \mathrm{Ti}$ \\
\hline${ }^{21} \mathrm{Ne}$ & $1.6 \times 10^{-4}$ & & ${ }^{21} \mathrm{Ne}$ \\
\hline${ }^{47} \mathrm{Cr}$ & $8.9 \times 10^{-5}$ & ${ }^{47} \mathrm{Ti}$ & \\
\hline
\end{tabular}

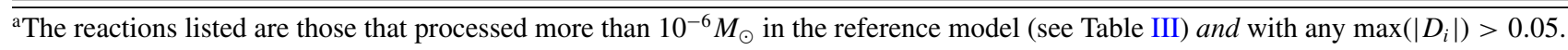

In the temperature range of interest, the rates given by these models match perfectly.

The rate of the reaction ${ }^{17} \mathrm{~F}+p \rightleftarrows{ }^{18} \mathrm{Ne}+\gamma$ is given in JINA for two different fits to experimental rates, which differ by more than one order of magnitude for temperatures in the range $10^{9} \lesssim T \lesssim 10^{10} \mathrm{~K}$. We have recomputed the nucleosynthesis with both evaluations of the rate of this reaction and show the results in the second row of Table XXII. Only the yield of ${ }^{14} \mathrm{~N}$, a marginal product of the supernova nucleosynthesis, changes by more than $10 \%$.

The reaction ${ }^{30} \mathrm{Si}+p \rightleftarrows{ }^{31} \mathrm{P}+\gamma$ has the largest $D_{i}$; consequently we have recomputed the nucleosynthesis using the three most recent evaluations of its rate from JINA. The results are shown in the third and fourth rows of Table XXII, in which we have taken as a reference the recommended rate from Ref. [59], which is compared to two other evaluations owing to Refs. [42] and [56]. In the temperature range of interest, the rates computed from these sources differ by less than a factor of 3 , and the same applies to the rate computed from the older Ref. [62]. The species whose yields are most sensitive to the different prescriptions for this rate are more or less the same as already noted in Tables VII and XV. However, the changes in the yields are more consistent with those shown in Table XV, indicating that the use of the enhancement factor function of temperature, as in Eq. (4), might describe better the actual uncertainties in the nucleosynthesis than using a fixed enhancement factor, at least at the current level of knowledge of this reaction rate (but see Secs. IV D and V). 

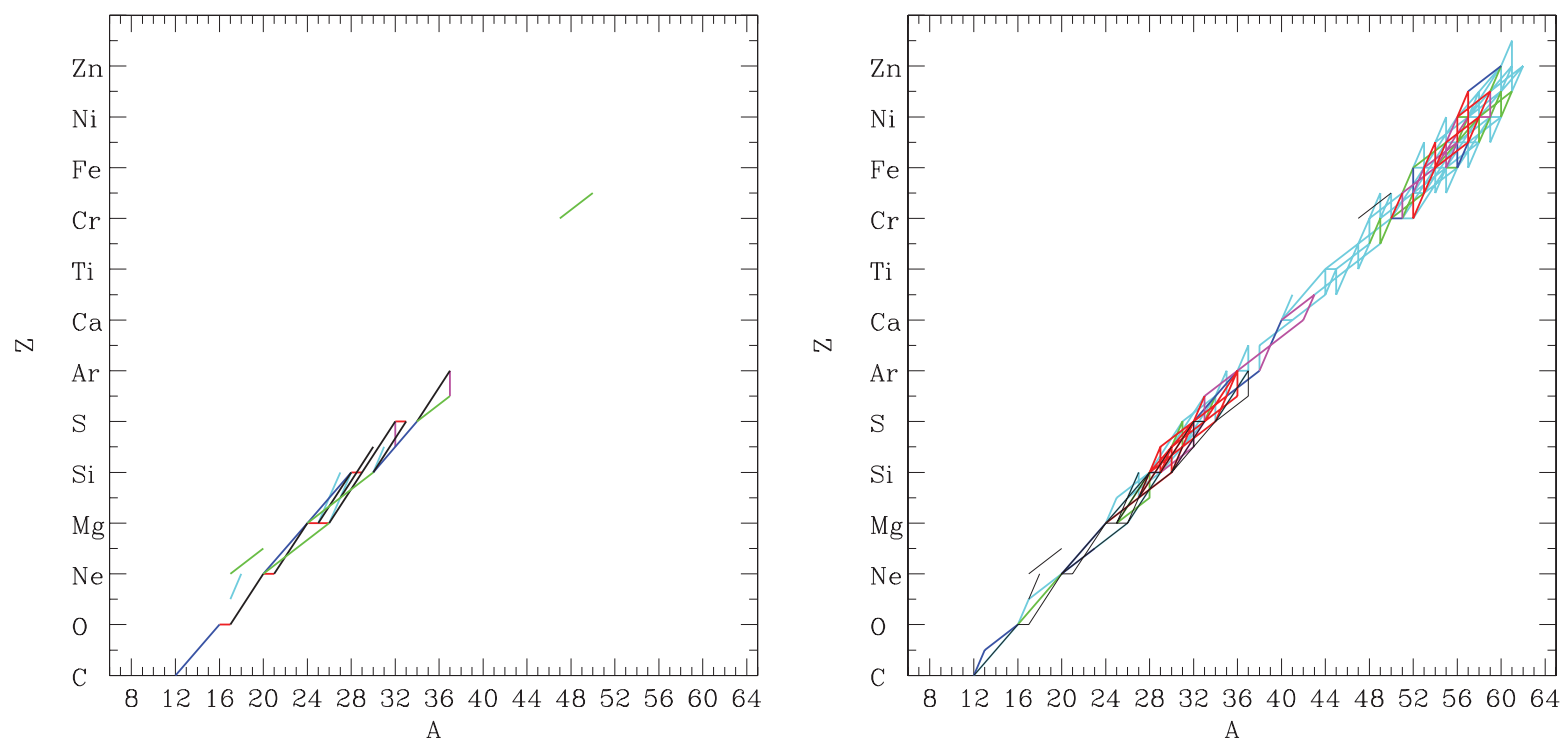

FIG. 12. (Color online) Chart of the most influential reactions compared to the maximum mass flow path. (Left) Reactions with max $\left(\left|D_{i}\right|\right)>$ 0.3 in Tables VI to XI. The type of each reaction can be deduced from the differences of atomic and baryonic number of the nuclei it connects or from the color in which they are drawn (online only): $(n, \gamma)$ reactions in red, $(p, \gamma)$ reactions in cyan, $(p, n)$ reactions in magenta, $(\alpha, \gamma)$ reactions in blue, $(\alpha, n)$ reactions in black, and $(\alpha, p)$ reactions in green. We recall that direct and inverse reaction rates were modified simultaneously. (Right) Reactions that carry a large mass flow. In this plot, the mass flow has been color coded (online version) according to the value of $\mathfrak{M}_{j k}$ : red for $\mathfrak{M}_{j k}>0.5 M_{\odot}$, magenta for $0.5 \geqslant \mathfrak{M}_{j k}>0.4 M_{\odot}$, blue for $0.4 \geqslant \mathfrak{M}_{j k}>0.3 M_{\odot}$, green for $0.3 \geqslant \mathfrak{M}_{j k}>0.2 M_{\odot}$, and cyan for $0.2 \geqslant \mathfrak{M}_{j k}>0.01 M_{\odot}$ (in the print version these lines appear as thick, successively lighter tones of gray). The chart of most influential reactions has been superimposed as black thin lines.

\section{3. $(p, n)$ reactions}

Only two $(p, n)$ reactions in Table VIII have any $\left|D_{i}\right|>$ 0.3 ; these are ${ }^{32} \mathrm{P}+p \rightleftarrows{ }^{32} \mathrm{~S}+n$ and ${ }^{37} \mathrm{Cl}+p \rightleftarrows{ }^{37} \mathrm{Ar}+n$. In the JINA library there are only theoretical rates of these reactions, all of them obtained from the NON-SMOKER code, using different nuclear inputs. In the range of temperatures of interest, the different rates for these reactions match each other perfectly.

\section{4. $(\alpha, \gamma)$ reactions}

The rates of the reactions ${ }^{24} \mathrm{Mg}+\alpha \rightleftarrows{ }^{28} \mathrm{Si}+\gamma$ and ${ }^{30} \mathrm{Si}+$ $\alpha \rightleftarrows{ }^{34} \mathrm{~S}+\gamma$ are derived from different evaluations obtained with the NON-SMOKER code with different nuclear inputs, and from Ref. [62]. All these rates, for a given reaction, agree within a factor smaller than the enhancement factor we have explored earlier in this paper; hence, we do not continue with the analysis of these two reactions.

The different prescriptions for the rate of the reaction ${ }^{20} \mathrm{Ne}+\alpha \rightleftarrows{ }^{24} \mathrm{Mg}+\gamma$ are from Refs. [42,56,60,62,67]. Within the temperature range of interest, these rates show discrepancies of nearly an order of magnitude. Thus, as this reaction is one of the most influential for SNIa nucleosynthesis, we have recomputed the yields for the three most recent prescriptions of its rate. The results are shown in the fifth and sixth rows in Table XXII. The list of species whose yields are most sensitive to the prescription used for this reaction is quite similar to that found in Table IX. The first point to note is the long list of species whose yield varies more than $10 \%$ when using either of the rates from Refs. [67], [42], or [60]. The species most sensitive to the changes in the ${ }^{20} \mathrm{Ne}+\alpha \rightleftarrows{ }^{24} \mathrm{Mg}+\gamma$ rates is ${ }^{24} \mathrm{Mg}$, whose yield changes by $156 \%$ when using the rate from Ref. [42] instead that from Ref. [67] and by $84 \%$ when using the rate from Ref. [60]. Several other species, such as ${ }^{27} \mathrm{Al}$ and ${ }^{30} \mathrm{Si}$, experience changes in the range $70 \%-90 \%$. The yields obtained using the theoretical rate in Ref. [56] (not shown in Table XXII) agree quite well with those obtained using the experimental rate in Ref. [60]. On the contrary, using the theoretical rates in Ref. [42], obtained with the same code as in Ref. [56] but with different nuclear inputs, gives yields that differ from those belonging to the rates from Ref. [60] by as much as $42 \%$.

The three most recent evaluations of the rate of the reaction ${ }^{12} \mathrm{C}+\alpha \rightleftarrows{ }^{16} \mathrm{O}+\gamma$ in JINA are from Refs. [60], [63], and [64]. We have recomputed the supernova nucleosynthesis for these three prescriptions of the rate. Not a single species experiences a change of abundance larger than $10 \%$.

The reaction ${ }^{58} \mathrm{Ni}+\alpha \rightleftarrows{ }^{62} \mathrm{Zn}+\gamma$ is important for the $\alpha$ rich freeze-out of NSE, which affects a large portion of SNIa ejecta. Hence, we have recomputed the nucleosynthesis with the three most recent rates given in JINA, from Ref. [42] and from Ref. [56] using different nuclear inputs. Not a single species experiences a change of abundance larger than $10 \%$.

\section{5. $(\alpha, n)$ reactions}

The different prescriptions for the reactions that have any $\left|D_{i}\right|>0.3$ in Table $\mathrm{X}$ give rates that agree with each other within the factor of 10 explored in this paper. These reactions are ${ }^{17} \mathrm{O}+\alpha \rightleftarrows{ }^{20} \mathrm{Ne}+n,{ }^{21} \mathrm{Ne}+\alpha \rightleftarrows{ }^{24} \mathrm{Mg}+n$, 
TABLE XII. Rates that influence the yields of each product species, from carbon to chlorine. ${ }^{\text {a }}$

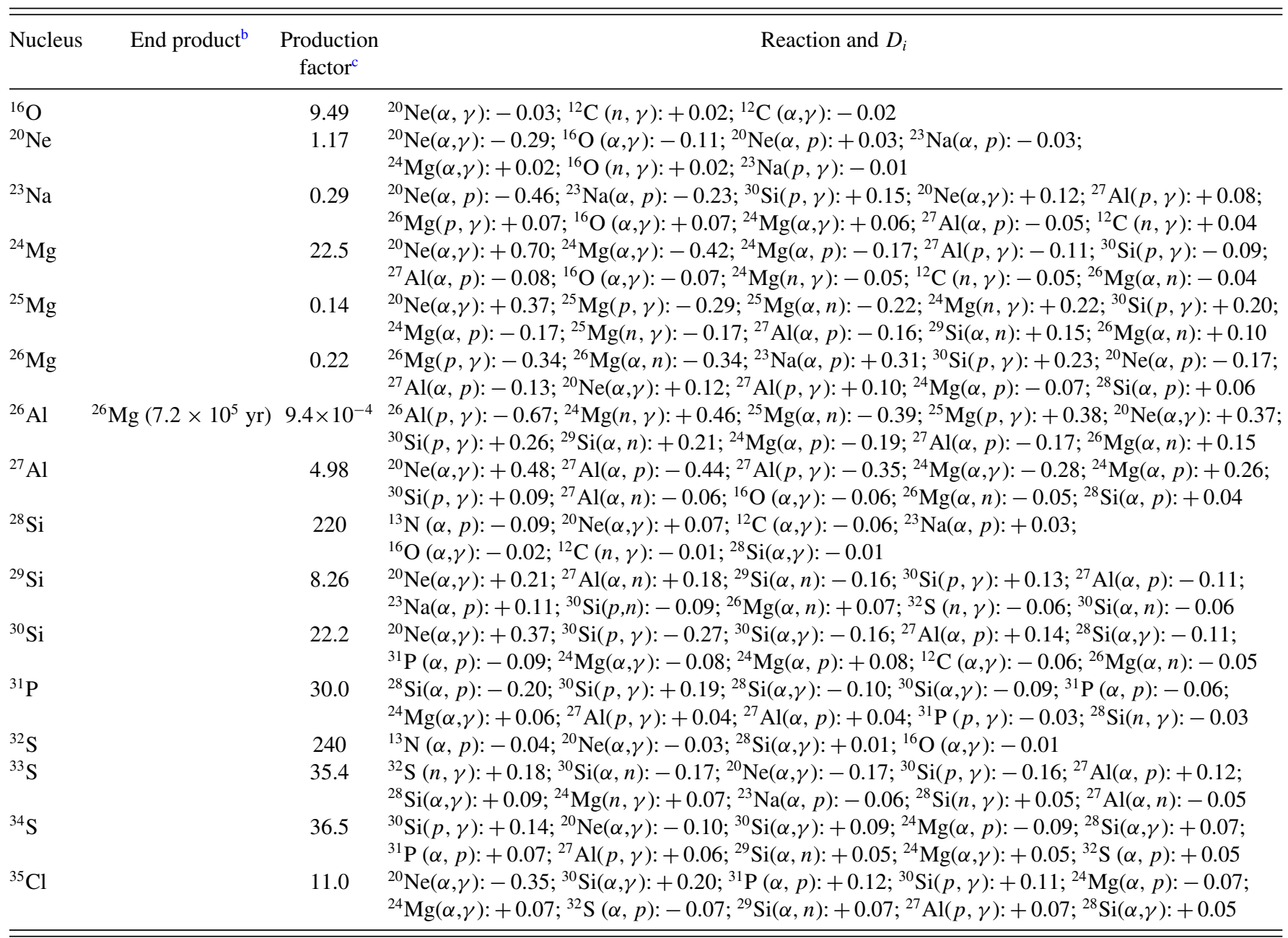

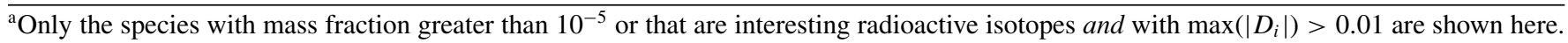
For each nucleus, we show a maximum of ten reactions.

${ }^{b}$ For radioactive nuclei, here is shown the end product of the disintegration chain as well as the longest half-life in the decay chain.

${ }^{\mathrm{c}}$ Mass fraction of the species in the supernova ejecta normalized to its solar mass fraction. For radioactive nuclides the normalization is with respect to the solar mass fraction of the end product of the disintegration chain.

${ }^{25} \mathrm{Mg}+\alpha \rightleftarrows{ }^{28} \mathrm{Si}+n,{ }^{26} \mathrm{Mg}+\alpha \rightleftarrows{ }^{29} \mathrm{Si}+n,{ }^{27} \mathrm{Al}+\alpha \rightleftarrows$ ${ }^{30} \mathrm{P}+n,{ }^{29} \mathrm{Si}+\alpha \rightleftarrows{ }^{32} \mathrm{~S}+n,{ }^{30} \mathrm{Si}+\alpha \rightleftarrows{ }^{33} \mathrm{~S}+n$, and ${ }^{34} \mathrm{~S}+$ $\alpha \rightleftarrows{ }^{37} \mathrm{Ar}+n$. The rates are from a variety of sources, most of them from calculations with the NON-SMOKER code using different nuclear inputs, but there are as well rates based on experimental measurements by Refs. $[60,62,65]$.

\section{6. $(\alpha, p)$ reactions}

The different prescriptions for the rates of the reactions ${ }^{17} \mathrm{Ne}+\alpha \rightleftarrows{ }^{20} \mathrm{Na}+p,{ }^{20} \mathrm{Ne}+\alpha \rightleftarrows{ }^{23} \mathrm{Na}+p,{ }^{23} \mathrm{Na}+\alpha \rightleftarrows$ ${ }^{26} \mathrm{Mg}+p,{ }^{27} \mathrm{Al}+\alpha \rightleftarrows{ }^{30} \mathrm{Si}+p,{ }^{34} \mathrm{~S}+\alpha \rightleftarrows{ }^{37} \mathrm{Cl}+p$, and ${ }^{47} \mathrm{Cr}+\alpha \rightleftarrows{ }^{50} \mathrm{Mn}+p$ all agree within a factor of 10 for the temperature range of interest to us. These reaction rates come from several calculations with the NON-SMOKER code using different nuclear inputs, as well as experimental measurements in Refs. $[60,65,66]$. The different rates of the reactions ${ }^{58} \mathrm{Ni}+$ $\alpha \rightleftarrows{ }^{61} \mathrm{Cu}+p$ and ${ }^{56} \mathrm{Ni}+\alpha \rightleftarrows{ }^{59} \mathrm{Cu}+p$, both of importance for the $\alpha$-rich freeze-out from NSE, agree quite well within the temperature range of interest. Thus, we do not continue with the analysis of these reaction rates.

The three most recent evaluations of the rate of the reaction ${ }^{24} \mathrm{Mg}+\alpha \rightleftarrows{ }^{27} \mathrm{Al}+p$ in the JINA database are from Refs. [42,59,61]. Within the temperature range of interest, the rate from the last reference differs from the other rates by as much as three orders of magnitude. Hence, we have recomputed the nucleosynthesis for the three different prescriptions of this rate. There is no significant difference in the chemical composition obtained with the rates of Refs. [42] and [59]; that is, not a single species experiences a change of abundance larger than $10 \%$, which is a consequence of the match between the rates from these two references. However, when comparing the yields obtained with the rate from Ref. [61] with those obtained with the rate from Ref. [59], many important variations in the yield of species with a significant abundance show up.

The three most recent evaluations of the rate of the reaction ${ }^{28} \mathrm{Si}+\alpha \rightleftarrows{ }^{31} \mathrm{P}+p$ in JINA are from the same references 
TABLE XIII. Rates that influence the yields of each product species, from argon to nickel (continuation of Table XII). ${ }^{\mathrm{a}}$

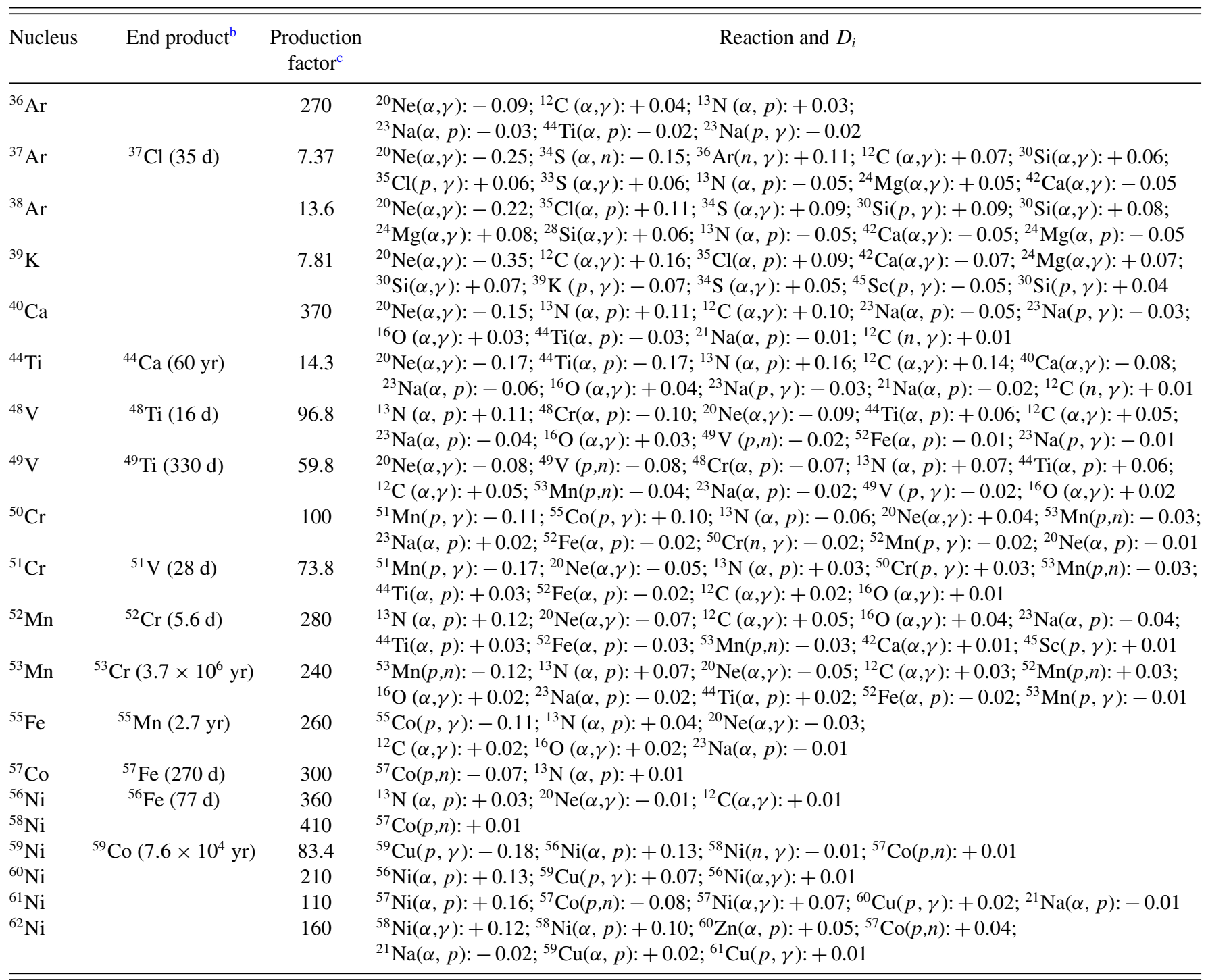

${ }^{\mathrm{a}}$ Only the species with mass fraction greater than $10^{-5}$ or that are interesting radioactive isotopes and with $\max \left(\left|D_{i}\right|\right)>0.01$ are shown here. For each nucleus, we show a maximum of ten reactions.

${ }^{b}$ For radioactive nuclei here is shown the end product of the disintegration chain as well as the longest half-life in the decay chain.

${ }^{\mathrm{c}}$ Mass fraction of the species in the supernova ejecta normalized to its solar mass fraction. For radioactive nuclides the normalization is with respect to the solar mass fraction of the end product of the disintegration chain.

as the reaction on ${ }^{24} \mathrm{Mg}$. Although the discrepancies of the rates are not as large as for $\mathrm{Mg}$, they reach one order of magnitude. Hence, we have recomputed as well the supernova nucleosynthesis for the three prescriptions of the rate of the reaction on ${ }^{28} \mathrm{Si}$. However, not a single species experiences a change of abundance larger than $10 \%$.

The reaction ${ }^{44} \mathrm{Ti}+\alpha \rightleftarrows{ }^{47} \mathrm{~V}+p$ is important for bridging the gap between QSE groups in silicon burning. Hence, we have recomputed the nucleosynthesis with the three most recent rates given in JINA, from Ref. [42] and from Ref. [56] using different nuclear inputs. Even though these rates differ up to near an order of magnitude in the range of temperatures of interest, not a single species experiences a change of abundance larger than $10 \%$.

\section{Summary of the sensitivity of the yields to different rate prescriptions}

The most notable reactions in Table XXII are ${ }^{30} \mathrm{Si}+p \rightleftarrows$ ${ }^{31} \mathrm{P}+\gamma,{ }^{20} \mathrm{Ne}+\alpha \rightleftarrows{ }^{24} \mathrm{Mg}+\gamma$, and ${ }^{24} \mathrm{Mg}+\alpha \rightleftarrows{ }^{27} \mathrm{Al}+p$. Within the set of most influential reactions and most influenced species, there are two important details. First, very few reactions appear whose parent nuclei belong to the Fe group or that are important for the bridging of the QSE groups in silicon burning. Second, in Table XXII there are no product species belonging to the Fe group whose yield depends significantly on the explored reaction rates. This is most remarkable because the elements of the Fe group constitute the main nucleosynthetic products of SNIa. 
TABLE XIV. Sensitivity of the nucleosynthesis to the rate of $(n, \gamma)$ reactions with the parent nuclide given in the first column, with enhancement factor given by Eq. (4). ${ }^{\mathrm{a}}$

\begin{tabular}{|c|c|c|}
\hline Parent nuclide & Nuclei with $\left|D_{i}\right|>0.3$ & Nuclei with $0.3>\left|D_{i}\right|>0.05$ \\
\hline${ }^{28} \mathrm{Si}$ & & ${ }^{21} \mathrm{Ne},{ }^{25} \mathrm{Mg},{ }^{26} \mathrm{Al},{ }^{35} \mathrm{~S}$ \\
\hline${ }^{55} \mathrm{Fe}$ & & ${ }^{55} \mathrm{Mn}$ \\
\hline${ }^{32} \mathrm{~S}$ & & ${ }^{32,33} \mathrm{P},{ }^{33,35} \mathrm{~S}$ \\
\hline${ }^{36} \mathrm{Ar}$ & & ${ }^{37} \mathrm{Cl},{ }^{37} \mathrm{Ar}$ \\
\hline${ }^{24} \mathrm{Mg}$ & & ${ }^{21} \mathrm{Ne},{ }^{25} \mathrm{Mg},{ }^{26} \mathrm{Al},{ }^{35} \mathrm{~S}$ \\
\hline${ }^{25} \mathrm{Mg}$ & & ${ }^{17} \mathrm{O},{ }^{21} \mathrm{Ne},{ }^{25} \mathrm{Mg},{ }^{26} \mathrm{Al}$ \\
\hline${ }^{56} \mathrm{Fe}$ & & ${ }^{57} \mathrm{Fe}$ \\
\hline${ }^{16} \mathrm{O}$ & & ${ }^{17} \mathrm{O}$ \\
\hline${ }^{46} \mathrm{Ti}$ & & ${ }^{46} \mathrm{Ti}$ \\
\hline${ }^{20} \mathrm{Ne}$ & ${ }^{21} \mathrm{Ne}$ & \\
\hline${ }^{12} \mathrm{C}$ & & ${ }^{21} \mathrm{Ne}$ \\
\hline${ }^{31} \mathrm{P}$ & & ${ }^{32} \mathrm{P}$ \\
\hline
\end{tabular}

${ }^{\text {a The reactions listed are those that processed more than } 10^{-6} M_{\odot} \text { in the reference model (see Table }}$ III) and with any $\max \left(\left|D_{i}\right|\right)>0.05$.

\section{Sensitivity to different temperature ranges}

As a final step in our present study, we now perform an analysis of the temperature dependence of the sensitivity of the yields to the reaction rates. We analyze here the three most notable rates found in Sec. IV C7. To this end, we consider again a fixed enhancement factor of the rates, a factor of 10 , but this time we limit it to a temperature window $10^{9} \mathrm{~K}$ wide. We explore windows centered on temperatures 1.5, 2.5, 3.5, $4.5,5.5$, and $6.5 \times 10^{9} \mathrm{~K}$. We show the results in Figs. 13 to 15 , where we plot for selected species the relative variation of their yield, defined as

$$
r_{i j}=\frac{m_{i j}-m_{i}}{m_{i}^{\prime}-m_{i}},
$$

where $m_{i}$ is the mass of nucleus $i$ ejected in our reference model (Table II), $m_{i}^{\prime}$ is the yield of the same species when the rate of the reaction being analyzed is multiplied by a factor of 10 independent of temperature, and $m_{i j}$ is the yield when the rate of the reaction is multiplied by the same factor only in the window $j \times 10^{9} \leqslant T \leqslant(j+1) \times 10^{9} \mathrm{~K}$. We selected the species to plot in the figures from among those with nonnegligible abundances that present a large difference between $m_{i}$ and $m_{i}^{\prime}$. We also required that the species covered a wide range of $Z$. Finally, we choose the same species to explore the sensitivities of all three reactions being considered: ${ }^{20} \mathrm{Ne}$, ${ }^{24} \mathrm{Mg},{ }^{26} \mathrm{Al},{ }^{30} \mathrm{Si},{ }^{32} \mathrm{P},{ }^{35} \mathrm{~S},{ }^{38} \mathrm{Ar}$, and ${ }^{47} \mathrm{Ti}$.

We show in Fig. 13 the results for the reaction ${ }^{30} \mathrm{Si}+p \rightleftarrows$ ${ }^{31} \mathrm{P}+\gamma$. We note that all the nuclei follow the same behavior

TABLE XV. Sensitivity of the nucleosynthesis to the rate of $(p, \gamma)$ reactions with the parent nuclide given in the first column, with enhancement factor given by Eq. (4). ${ }^{\mathrm{a}}$

\begin{tabular}{|c|c|c|}
\hline Parent nuclide & Nuclei with $\left|D_{i}\right|>0.3$ & Nuclei with $0.3>\left|D_{i}\right|>0.05$ \\
\hline${ }^{29} \mathrm{Si}$ & & ${ }^{26} \mathrm{Al},{ }^{35} \mathrm{~S},{ }^{43} \mathrm{Ca}$ \\
\hline${ }^{35} \mathrm{Cl}$ & & ${ }^{37} \mathrm{Cl}$ \\
\hline${ }^{30} \mathrm{Si}$ & ${ }^{35} \mathrm{~S}$ & ${ }^{23} \mathrm{Na},{ }^{25,26} \mathrm{Mg},{ }^{26} \mathrm{Al},{ }^{29,30} \mathrm{Si}^{31,32} \mathrm{P},{ }^{33,34} \mathrm{~S},{ }^{35} \mathrm{Cl},{ }^{43} \mathrm{Ca},{ }^{47} \mathrm{Ti}$ \\
\hline${ }^{27} \mathrm{Al}$ & & ${ }^{23} \mathrm{Na},{ }^{24-26} \mathrm{Mg},{ }^{26,27} \mathrm{Al},{ }^{32} \mathrm{P},{ }^{35} \mathrm{~S},{ }^{43} \mathrm{Ca},{ }^{47} \mathrm{Ti}$ \\
\hline${ }^{59} \mathrm{Cu}$ & & ${ }^{59} \mathrm{Ni}$ \\
\hline${ }^{56} \mathrm{Co}$ & & ${ }^{56} \mathrm{Co}$ \\
\hline${ }^{33} \mathrm{~S}$ & & ${ }^{43} \mathrm{Ca}$ \\
\hline${ }^{51} \mathrm{Mn}$ & & ${ }^{50,51} \mathrm{Cr}$ \\
\hline${ }^{47} \mathrm{~V}$ & & ${ }^{47} \mathrm{Ti}$ \\
\hline${ }^{26} \mathrm{Al}$ & ${ }^{26} \mathrm{Al}$ & \\
\hline${ }^{59} \mathrm{Co}$ & & ${ }^{59} \mathrm{Co}$ \\
\hline${ }^{26} \mathrm{Mg}$ & & ${ }^{26} \mathrm{Mg},{ }^{26} \mathrm{Al},{ }^{35} \mathrm{~S},{ }^{43} \mathrm{Ca},{ }^{47} \mathrm{Ti}$ \\
\hline${ }^{25} \mathrm{Mg}$ & & ${ }^{21} \mathrm{Ne},{ }^{25} \mathrm{Mg},{ }^{26} \mathrm{Al},{ }^{35} \mathrm{~S}$ \\
\hline${ }^{58} \mathrm{Fe}$ & & ${ }^{58} \mathrm{Fe}$ \\
\hline${ }^{45} \mathrm{Sc}$ & & ${ }^{45} \mathrm{Sc}$ \\
\hline${ }^{62} \mathrm{Cu}$ & & ${ }^{63} \mathrm{Cu},{ }^{65} \mathrm{Zn}$ \\
\hline${ }^{37} \mathrm{Cl}$ & & ${ }^{37} \mathrm{Cl}$ \\
\hline${ }^{17} \mathrm{~F}$ & & ${ }^{14} \mathrm{~N}$ \\
\hline${ }^{64} \mathrm{Ga}$ & & ${ }^{63} \mathrm{Cu},{ }^{65} \mathrm{Zn}$ \\
\hline
\end{tabular}

${ }^{a}$ The reactions listed are those that processed more than $10^{-6} M_{\odot}$ in the reference model (see Table III) and with any $\max \left(\left|D_{i}\right|\right)>0.05$. 
TABLE XVI. Sensitivity of the nucleosynthesis to the rate of $(p, n)$ reactions with the parent nuclide given in the first column, with enhancement factor given by Eq. (4). ${ }^{\text {a }}$

\begin{tabular}{llll}
\hline \hline Parent nuclide & Nuclei with $\left|D_{i}\right|>0.05$ & Parent nuclide & Nuclei with $\left|D_{i}\right|>0.05$ \\
\hline${ }^{53} \mathrm{Mn}$ & ${ }^{53} \mathrm{Mn}$ & ${ }^{32} \mathrm{P}$ & ${ }^{32} \mathrm{P}$ \\
${ }^{30} \mathrm{Si}$ & ${ }^{35} \mathrm{~S}$ & ${ }^{45} \mathrm{Sc}$ & ${ }^{45} \mathrm{Sc}$ \\
${ }^{58} \mathrm{Co}$ & ${ }^{58} \mathrm{Co}$ & ${ }^{37} \mathrm{Cl}$ & ${ }^{37} \mathrm{Cl}$ \\
${ }^{54} \mathrm{Mn}$ & ${ }^{54} \mathrm{Mn}$ & ${ }^{62} \mathrm{Cu}$ & ${ }^{63} \mathrm{Cu},{ }^{65} \mathrm{Zn}$ \\
${ }^{55} \mathrm{Mn}$ & ${ }^{55} \mathrm{Mn}$ & ${ }^{47} \mathrm{Ti}$ & ${ }^{46} \mathrm{Ti}$ \\
\hline
\end{tabular}

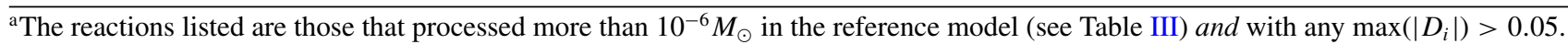

with respect to the temperature window in which the reaction rate is modified. There is a modest variation of order $20 \%$ of the yields for the window centered on $1.5 \times 10^{9} \mathrm{~K}$ and a large increase for the next window, centered on $2.5 \times 10^{9} \mathrm{~K}$. In the window centered on $3.5 \times 10^{9} \mathrm{~K}$ there are some species that experience large variations of their yields while others are scarcely affected at all. The yield of ${ }^{20} \mathrm{Ne}$ (open triangles) shows a peculiar behavior, with $r_{i j}<0$ in the first thermal window, meaning that increasing the rate of the reaction only at low temperatures $\left(T \leqslant 2 \times 10^{9} \mathrm{~K}\right)$ results in a variation of the yield of ${ }^{20} \mathrm{Ne}$ of opposite sign as that obtained if the reaction rate is increased for any temperature. Note that the sign of $r_{i j}$ of ${ }^{20} \mathrm{Ne}$ in the next window is positive, and it has the largest $r_{i j}$ among the species shown in the figure: Increasing the reaction rate only in the interval $2 \times 10^{9} \leqslant T \leqslant 3 \times 10^{9} \mathrm{~K}$ produces a change of the yield of this species that is as much as that obtained by increasing the reaction rate for all temperatures. Modifying the rate on thermal windows above $4 \times 10^{9} \mathrm{~K}$ has no effect on any of the final abundances of the species.

We show as well in Fig. 13 the ratio of the rates belonging to the three prescriptions adopted for the rate of the reaction ${ }^{30} \mathrm{Si}+p \rightleftarrows{ }^{31} \mathrm{P}+\gamma$, which were discussed in Sec. IV C2 and in Table XXII. The uncertainty in the rates derived from these different prescriptions is more or less uniform for temperatures above $\sim 2 \times 10^{9} \mathrm{~K}$. The rate from Ref. [56] differs most from that based on Ref. [59] at temperatures where the yields are most sensitive to this reaction rate. However, the discrepancy between these rates is much lower than the factor of 10 used in our simulations; thus, we believe that the supernova yields should not be affected by any reasonable future change of this reaction rate.

Figure 14 summarizes the results for the reaction ${ }^{20} \mathrm{Ne}+$ $\alpha \rightleftarrows{ }^{24} \mathrm{Mg}+\gamma$. The most noticeable difference with respect to Fig. 13 is the behavior and range of the variations of the yield of ${ }^{38} \mathrm{Ar}$ (asterisks). The maximum sensitivity of this species occurs in the temperature window $4 \times 10^{9} \leqslant T \leqslant 5 \times 10^{9} \mathrm{~K}$, where the change of its yield reaches a value seven times larger than the change with a rate modified at all temperatures. This is compensated by the fact that modifying the rate at temperatures in the interval $2 \times 10^{9} \leqslant T \leqslant 4 \times 10^{9} \mathrm{~K}$ produces a change of the yield of ${ }^{38} \mathrm{Ar}$ of opposite sign. The rest of nuclei plotted show a behavior similar to the one in Fig. 13, with maximum $\left|r_{i j}\right| \sim 1.5$ ( ${ }^{32} \mathrm{P}$, solid pentagons).

As revealed by Fig. 14, the different prescriptions for the rate of the reaction ${ }^{20} \mathrm{Ne}+\alpha \rightarrow{ }^{24} \mathrm{Mg}+\gamma$ show a maximum discrepancy by a factor of $\sim 10$ in the temperature range $10^{9}-$ $10^{10} \mathrm{~K}$. However, both the rate from Ref. [42] and that from Ref. [60] differ from the rate given in Ref. [67] by a similar factor in the interval $2 \times 10^{9} \leqslant T \leqslant 4 \times 10^{9} \mathrm{~K}$.

Finally, we show in Fig. 15 the results for the reaction ${ }^{24} \mathrm{Mg}+\alpha \rightleftarrows{ }^{27} \mathrm{Al}+p$. It highlights the behavior of ${ }^{30} \mathrm{Si}$ (open

TABLE XVII. Sensitivity of the nucleosynthesis to the rate of $(\alpha, \gamma)$ reactions with the parent nuclide given in the first column, with enhancement factor given by Eq. (4). ${ }^{\mathrm{a}}$

\begin{tabular}{|c|c|c|}
\hline Parent nuclide & Nuclei with $\left|D_{i}\right|>0.3$ & Nuclei with $0.3>\left|D_{i}\right|>0.05$ \\
\hline${ }^{28} \mathrm{Si}$ & & ${ }^{30} \mathrm{Si}$ \\
\hline${ }^{32} \mathrm{~S}$ & & ${ }^{37} \mathrm{Cl}$ \\
\hline${ }^{16} \mathrm{O}$ & & ${ }^{21} \mathrm{Ne},{ }^{23} \mathrm{Na}$ \\
\hline${ }^{24} \mathrm{Mg}$ & ${ }^{43} \mathrm{Ca}$ & ${ }^{24} \mathrm{Mg},{ }^{27} \mathrm{Al},{ }^{35} \mathrm{~S},{ }^{47} \mathrm{Ti}$ \\
\hline${ }^{12} \mathrm{C}$ & & ${ }^{39} \mathrm{~K},{ }^{41,42} \mathrm{Ca},{ }^{45} \mathrm{Sc},{ }^{44,46} \mathrm{Ti}$ \\
\hline${ }^{33} \mathrm{~S}$ & & ${ }^{37} \mathrm{Cl}$ \\
\hline${ }^{30} \mathrm{Si}$ & & ${ }^{30} \mathrm{Si},{ }^{32,33} \mathrm{P},{ }^{34,35} \mathrm{~S},{ }^{35} \mathrm{Cl}$ \\
\hline${ }^{41} \mathrm{Ca}$ & & ${ }^{43} \mathrm{Ca},{ }^{47} \mathrm{Ti}$ \\
\hline${ }^{42} \mathrm{Ca}$ & & ${ }^{46} \mathrm{Ti}$ \\
\hline${ }^{62} \mathrm{Zn}$ & & ${ }^{66} \mathrm{Zn}$ \\
\hline
\end{tabular}

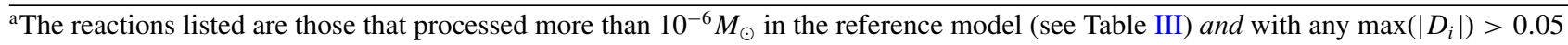


TABLE XVIII. Sensitivity of the nucleosynthesis to the rate of $(\alpha, n)$ reactions with the parent nuclide given in the first column, with enhancement factor given by Eq. (4). ${ }^{\mathrm{a}}$

\begin{tabular}{lcc}
\hline \hline Parent nuclide & Nuclei with $\left|D_{i}\right|>0.3$ & Nuclei with $0.3>\left|D_{i}\right|>0.05$ \\
\hline${ }^{29} \mathrm{Si}$ & & ${ }^{25} \mathrm{Mg},{ }^{26} \mathrm{Al},{ }^{29} \mathrm{Si},{ }^{35} \mathrm{~S},{ }^{43} \mathrm{Ca}$ \\
${ }^{33} \mathrm{Sa}$ & ${ }^{37} \mathrm{Cl},{ }^{43} \mathrm{Ca}$ \\
${ }^{27} \mathrm{Al}$ & ${ }^{43} \mathrm{Ca}$ & ${ }^{25} \mathrm{Mg},{ }^{29} \mathrm{Si},{ }^{32,33} \mathrm{P},{ }^{35} \mathrm{~S},{ }^{47} \mathrm{Ti}$ \\
${ }^{30} \mathrm{Si}$ & & $33 \mathrm{P},{ }^{33,35} \mathrm{~S},{ }^{37} \mathrm{Cl},{ }^{43} \mathrm{Ca},{ }^{47} \mathrm{Ti}$ \\
${ }^{25} \mathrm{Mg}$ & & ${ }^{17} \mathrm{O},{ }^{21} \mathrm{Ne},{ }^{25} \mathrm{Mg},{ }^{26} \mathrm{Al},{ }^{35} \mathrm{~S}$ \\
${ }^{34} \mathrm{~S}$ & & ${ }^{37} \mathrm{Cl},{ }^{37} \mathrm{Ar}$ \\
${ }^{26} \mathrm{Mg}$ & ${ }^{21} \mathrm{Ne},{ }^{25,26} \mathrm{Mg},{ }^{26} \mathrm{Al},{ }^{32} \mathrm{P},{ }^{35} \mathrm{~S},{ }^{43} \mathrm{Ca},{ }^{47} \mathrm{Ti}$ \\
${ }^{41} \mathrm{Ca}$ & ${ }^{43} \mathrm{Ca},{ }^{47} \mathrm{Ti}$ \\
${ }^{21} \mathrm{Ne}$ & ${ }^{21} \mathrm{Ne}$ & \\
${ }^{17} \mathrm{O}$ & & ${ }^{17} \mathrm{O},{ }^{21} \mathrm{Ne}$ \\
\hline \hline
\end{tabular}

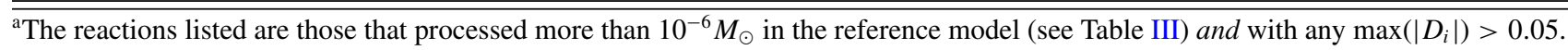

pentagons), whose yield experiences variations up to seven times larger in the temperature window $3 \times 10^{9} \leqslant T \leqslant$ $4 \times 10^{9} \mathrm{~K}$ than when the rate is modified for all temperatures. Furthermore, the variation of its yield changes sign if the thermal window is $2 \times 10^{9} \leqslant T \leqslant 3 \times 10^{9} \mathrm{~K}$, still reaching $\left|r_{i j}\right| \sim 7$. The rest of the nuclei show a behavior similar to that in Fig. 13, although their maximum $\left|r_{i j}\right|$ is now a bit larger, $\max \left(\left|r_{i j}\right|\right) \sim 2$.

The ratio of the rates of the reaction ${ }^{24} \mathrm{Mg}+\alpha \rightleftarrows{ }^{27} \mathrm{Al}+p$ from Refs. [42] and [59] agree quite well for $T \gtrsim 2 \times 10^{9} \mathrm{~K}$ (see Fig. 15). However, the rate from Ref. [61] differs from the other two by more than two orders of magnitude at high temperatures. However, in the range of temperatures where the abundances plotted are most sensitive to this reaction, $2 \times 10^{9} \leqslant T \leqslant 4 \times 10^{9} \mathrm{~K}$, their discrepancy is less than a factor of $\sim 30$. It is interesting to note that, using these rates, the change of the sensitivity of the yield of ${ }^{30} \mathrm{Si}$ in the two temperature windows with $\left|r_{i j}\right| \sim 7$ almost compensates each other, with the result that the final yield of this species is negligibly affected by using the rate from Ref. [61] instead of that from Ref. [59] (thus, it does not appear in the row reserved for this reaction in Table XXII).

\section{CONCLUSIONS}

We have computed the chemical composition of a reference SNIa model with a nucleosynthetic postprocessing code that takes as inputs the nuclear data and the thermodynamic trajectories of each mass shell. Our reference SNIa model is the onedimensional delayed-detonation model DDTc in Ref. [48]. Our nucleosynthetic calculations include 3138 reactions during the integration of the nuclear evolutionary equations but only 1096 of them can contribute significantly to the nucleosynthesis of the supernova model. In this paper, we have explored the sensitivity of the SNIa explosive nucleosynthesis to simple variations on nuclear reaction rates (either a fixed enhancement factor or one that decreases monotonously with temperature) and comparisons between different theoretical and experimental prescriptions of the rates.

The nucleosynthesis resulting from our type Ia supernova model is quite robust with respect to variations of nuclear reaction rates, with the exception of the fusion of two ${ }^{12} \mathrm{C}$ nuclei. The energy of the explosion changes by less than $\sim 4 \%$ when the rates of the reactions ${ }^{12} \mathrm{C}+{ }^{12} \mathrm{C}$ or ${ }^{16} \mathrm{O}+{ }^{16} \mathrm{O}$ are multiplied by a factor of 10 or 0.1 . The changes in the

TABLE XIX. Sensitivity of the nucleosynthesis to the rate of $(\alpha, p)$ reactions with the parent nuclide given in the first column, with enhancement factor given by Eq. (4). ${ }^{\mathrm{a}}$

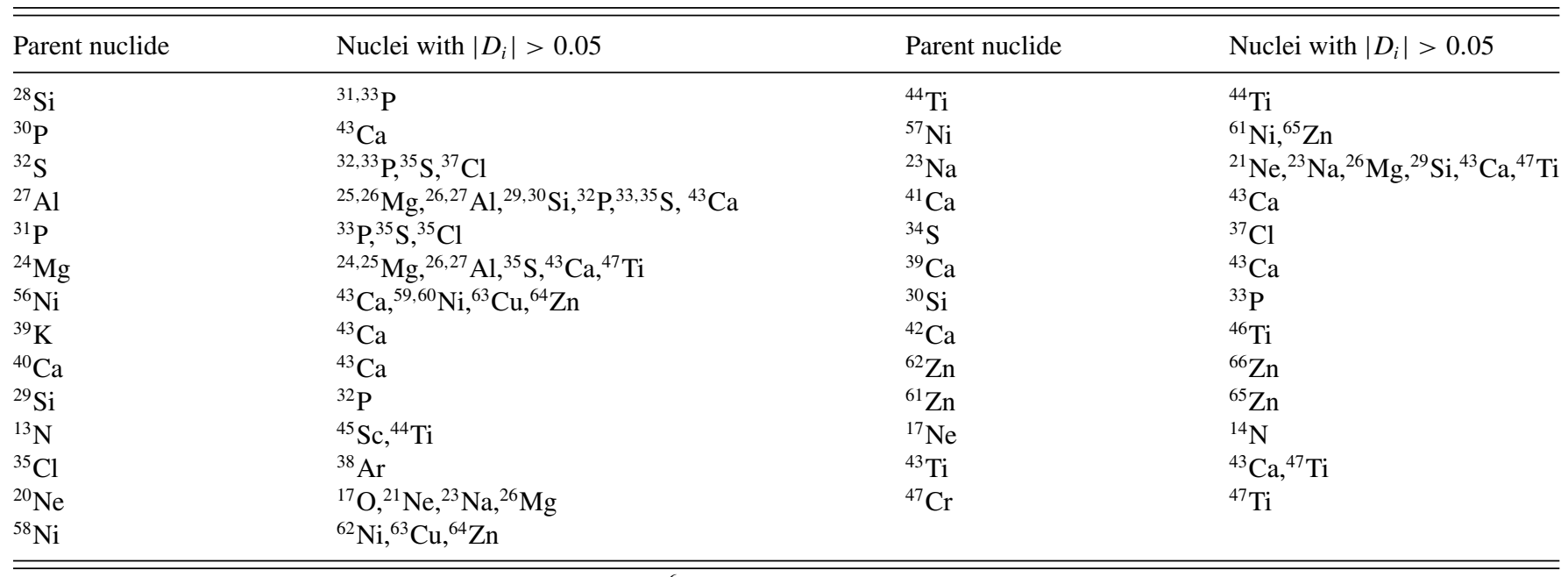

${ }^{\mathrm{a}}$ The reactions listed are those that processed more than $10^{-6} M_{\odot}$ in the reference model (see Table III) and with any max $\left(\left|D_{i}\right|\right)>0.05$. 










TABLE XXII. Sensitivity of the nucleosynthesis to different rate prescriptions for the reactions in the first column.

\begin{tabular}{|c|c|}
\hline Reaction & Product nuclei and percent variation ${ }^{\mathrm{a}}$ \\
\hline${ }^{16} \mathrm{O}+n \rightleftarrows{ }^{17} \mathrm{O}+\gamma^{\mathrm{b}}$ & ${ }^{17} \mathrm{O}:-99 \% ;{ }^{14} \mathrm{~N}:-27 \% ;{ }^{25} \mathrm{Mg}:-25 \% ;{ }^{22} \mathrm{Ne}:+22 \% ;{ }^{43} \mathrm{Ca}:+18 \% ;{ }^{21} \mathrm{Ne}:+15 \% ;{ }^{35} \mathrm{~S}:+14 \% ;{ }^{26} \mathrm{Al}:-11 \%$ \\
\hline${ }^{17} \mathrm{~F}+p \rightleftarrows{ }^{18} \mathrm{Ne}+\gamma^{\mathrm{c}}$ & ${ }^{14} \mathrm{~N}:+79 \%$ \\
\hline${ }^{30} \mathrm{Si}+p \rightleftarrows{ }^{31} \mathrm{P}+\gamma^{\mathrm{e}}$ & $\begin{array}{l}{ }^{35} \mathrm{~S}:+68 \% ;{ }^{32} \mathrm{P}:+26 \% ;{ }^{43} \mathrm{Ca}:+24 \% ;{ }^{30} \mathrm{Si}:-22 \% ;{ }^{26} \mathrm{Al}:+21 \% ;{ }^{25} \mathrm{Mg}:+20 \% ;{ }^{29} \mathrm{Si}:+16 \% ;{ }^{26} \mathrm{Mg}:+16 \% \\
{ }^{33} \mathrm{~S}:-14 \% ;{ }^{31} \mathrm{P}:+13 \% ;{ }^{34} \mathrm{~S}:+12 \%\end{array}$ \\
\hline${ }^{20} \mathrm{Ne}+\alpha \rightleftarrows{ }^{24} \mathrm{Mg}+\gamma^{\mathrm{g}}$ & $\begin{array}{l}{ }^{24} \mathrm{Mg}:+84 \% ;{ }^{40} \mathrm{~K}:-58 \% ;{ }^{43} \mathrm{Ca}:-56 \% ;{ }^{27} \mathrm{Al}:+52 \% ;{ }^{48} \mathrm{Ti}:-51 \% ;{ }^{30} \mathrm{Si}:+48 \% ;{ }^{44} \mathrm{Ca}:-48 \% ;{ }^{35} \mathrm{~S}:-37 \% \\
{ }^{42} \mathrm{Ca}:-37 \% ;{ }^{45} \mathrm{Sc}:-37 \% ;{ }^{33} \mathrm{P}:+36 \% ;{ }^{36} \mathrm{~S}:+35 \% ;{ }^{35} \mathrm{Cl}:-35 \% ;{ }^{47} \mathrm{Ti}:-32 \% ;{ }^{44} \mathrm{Ti}:-30 \% ;{ }^{26} \mathrm{Al}:+29 \% \\
{ }^{32} \mathrm{P}:+29 \% ;{ }^{38} \mathrm{Ar}:-29 \% ;{ }^{11} \mathrm{Ca}:-28 \% ;{ }^{39} \mathrm{~K}:-26 \% ;{ }^{46} \mathrm{Ti}:-26 \% ;{ }^{37} \mathrm{Ar}:-24 \% ;{ }^{25} \mathrm{Mg}:+21 \% ;{ }^{29} \mathrm{Si}:+21 \% \\
{ }^{34} \mathrm{~S}:-20 \% ;{ }^{40} \mathrm{Ca}:-19 \% ;{ }^{20} \mathrm{Ne}:-16 \% ;{ }^{33} \mathrm{~S}:-16 \% ;{ }^{14} \mathrm{~N}:-15 \% ;{ }^{37} \mathrm{Cl}:-14 \% ;{ }^{28} \mathrm{Si}:+12 \% ;{ }^{48} \mathrm{~V}:-11 \%\end{array}$ \\
\hline
\end{tabular}

${ }^{\mathrm{a}}$ We only show species for which the percent variation is largest than $10 \%$ in absolute value and whose yield is $\gtrsim 10^{-8} M_{\odot}$.

${ }^{\mathrm{b}}$ Rate from Ref. [57] vs rate from Ref. [55].

${ }^{\mathrm{c}}$ The reference rate from Ref. [58] is compared to a rate that incorporates several contributions from M. Wiescher, as given in the JINA Database.

${ }^{\mathrm{d}}$ Rate from Ref. [42] vs rate from Ref. [59].

${ }^{\mathrm{e}}$ Rate from Ref. [56] vs rate from Ref. [59].

${ }^{\mathrm{f}}$ Rate from Ref. [42] vs rate from Ref. [67].

${ }^{g}$ Rate from Ref. [60] vs rate from Ref. [67].

${ }^{\mathrm{h}}$ Rate from Ref. [61] vs rate from Ref. [59].

nucleosynthesis owing to the modification of the rates of these fusion reactions are also quite modest; for instance, no species with a mass fraction larger than 0.02 experiences a variation of its yield larger than a factor of 2 . The robustness of the production of ${ }^{56} \mathrm{Ni}$ and many other Fe-group isotopes stands out. If the enhancement factor of the rates is a decreasing function of temperature, the effect on the yields of all the species is even less than with a fixed enhancement factor. For instance, no species experiences a variation of its yield larger than $30 \%$ with respect to the rate of ${ }^{16} \mathrm{O}+{ }^{16} \mathrm{O}$ or with respect to the $3 \alpha$ rate. We have checked that the modifications in the nucleosynthesis produced when the fusion rates are modified only in the nucleosynthetic code are quite similar to those obtained when the rates are modified in the full supernova simulation.

We provide the sensitivities of the yield of each relevant nuclear species ejected in the supernova with respect to those nuclear reactions that affect it. In general, the yields of Fegroup nuclei are less sensitive than the yields of intermediatemass elements. However, the yields of ${ }^{28} \mathrm{Si}$ and ${ }^{32} \mathrm{~S}$, as well as ${ }^{54} \mathrm{Fe},{ }^{56} \mathrm{Ni}$, and ${ }^{58} \mathrm{Ni}$, do not change appreciably within the range of enhancement factors of the nuclear reaction rates explored here. The only reactions for which the relative change of the abundance of any species is larger (in absolute value) than the relative change in the rate $\left(\left|D_{i}\right|>1\right)$ is ${ }^{30} \mathrm{Si}+p \rightleftarrows$ ${ }^{31} \mathrm{P}+\gamma$. In general, radiative captures of protons are the group of reactions with the largest influence on the supernova yields. Other important groups of reactions are radiative captures of $\alpha$ particles, most notably the reactions ${ }^{20} \mathrm{Ne}+\alpha \rightleftarrows{ }^{24} \mathrm{Mg}+$ $\gamma$, for which there are 33 species whose yields change by more than $12 \%$, and the $(\alpha, p)$ reactions ${ }^{13} \mathrm{~N}+\alpha \rightleftarrows{ }^{16} \mathrm{O}+p$, ${ }^{20} \mathrm{Ne}+\alpha \rightleftarrows{ }^{23} \mathrm{Na}+p,{ }^{23} \mathrm{Na}+\alpha \rightleftarrows{ }^{26} \mathrm{Mg}+p,{ }^{24} \mathrm{Mg}+\alpha \rightleftarrows$ ${ }^{27} \mathrm{Al}+p$, and ${ }^{27} \mathrm{Al}+\alpha \rightleftarrows{ }^{30} \mathrm{Si}+p$.

We have discussed the sensitivity of the nucleosynthesis to the rates of reactions that take part in the most relevant nucleosynthetic processes in SNIa. Modifying the rates of the reactions that bridge the gap between QSE groups in explosive silicon burning has a very limited effect on the yields. They affect mainly nuclei that belong to this gap. Changing the rate of reactions relevant for the $\alpha$-rich freeze-out does not produce important changes on the abundances of nuclear species either. This can be explained both by the small amount of matter that goes through $\alpha$-rich freeze-out from NSE and by the small excess of $\alpha$ particles at freeze-out (see Fig. 1, where the maximum value of $Y_{\alpha}$ does not attain 0.01 $\left.\mathrm{mol} \mathrm{g}^{-1}\right)$. It is as well remarkable that the reactions involving nuclei with $Z>22$ have a tiny influence on the supernova nucleosynthesis.

We have relied on the JINA REACLIB Database to estimate realistic uncertainties of the most relevant reaction rates, by comparing the most recent prescriptions for the rates of these reactions. We have paid special attention to the reactions ${ }^{30} \mathrm{Si}+p \rightleftarrows{ }^{31} \mathrm{P}+\gamma,{ }^{20} \mathrm{Ne}+\alpha \rightleftarrows{ }^{24} \mathrm{Mg}+\gamma$, and ${ }^{24} \mathrm{Mg}+\alpha \rightleftarrows{ }^{27} \mathrm{Al}+p$, especially the last one for which there is a discrepancy of up to three orders of magnitude between the rates owing to Refs. [59] and [61]. In spite of this large difference of rates, the maximum change in the yields is only $52 \%$, belonging to ${ }^{35} \mathrm{~S}$. 


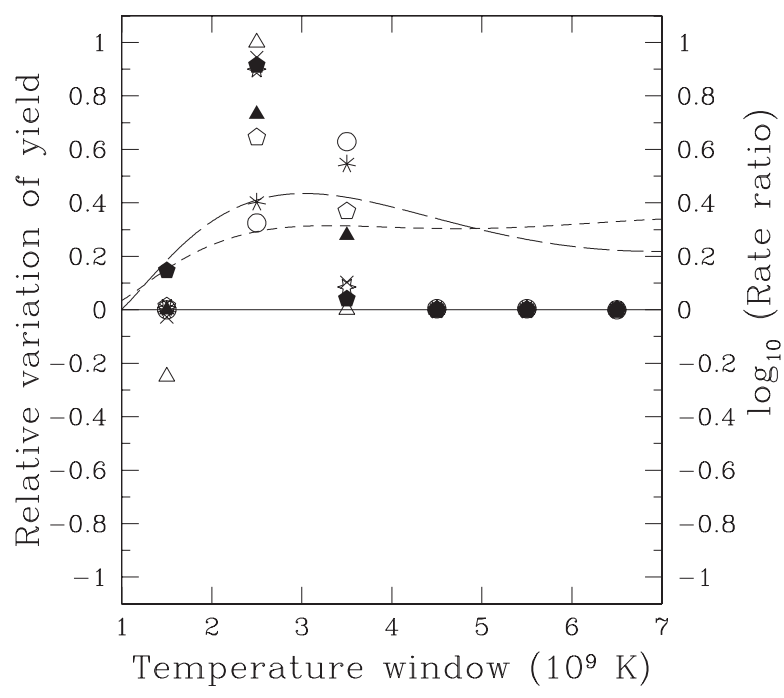

FIG. 13. Sensitivity of the yield of selected species to variations by a factor of $\times 10$ in the rate of the reactions ${ }^{30} \mathrm{Si}+p \rightleftarrows{ }^{31} \mathrm{P}+\gamma$ in different temperature ranges of size $10^{9} \mathrm{~K}$. The points give the difference between the yield of a species for an enhanced rate in a temperature window and its yield in our reference model, normalized by the difference between the yield for an enhanced rate at all temperatures and the yield of our reference model [see Eq. (6)]. The points are centered on each temperature window, and each symbol represents a product nucleus as follows: Open triangles stand for ${ }^{20} \mathrm{Ne}$, solid triangles for ${ }^{24} \mathrm{Mg}$, crosses for ${ }^{26} \mathrm{Al}$, open pentagons for ${ }^{30} \mathrm{Si}$, solid pentagons for ${ }^{32} \mathrm{P}$, stars for ${ }^{35} \mathrm{~S}$, asterisks with seven vertices for ${ }^{38} \mathrm{Ar}$, and open circles for ${ }^{47} \mathrm{Ti}$. The dashed lines (scaled according to the right axis) give the logarithm of the ratio of the different rates of the reaction ${ }^{30} \mathrm{Si}+p \rightarrow{ }^{31} \mathrm{P}+\gamma$ in JINA. The short-dashed line belongs to the ratio of the rate from Ref. [42] to that from Ref. [59], while the long-dashed line belongs to the ratio of the rate from Ref. [56] to that from Ref. [59]. The horizontal solid line marks the zero of axes, that is, no variation of the yield and rate ratio equal to one.

There are two main reasons for the small relative impact of the uncertainties of individual nuclear reaction rates on the supernova yields. First, the nuclear flows that determine the final abundances during the supernova explosion are driven collectively by many reactions, which are much faster than the hydrodynamic explosion time scale because of the high temperatures involved. The relevance of any individual rate is much diluted within this large pool of reactions. A similar conclusion was reached by Ref. [52] in the context of type II supernovae. They cite three major causes, which we can adapt to nucleosynthesis in SNIa. (1) The dominant nuclear flows are governed by the fusion reactions of the fuel, carbon and oxygen, while the rest are only perturbations on the main stream. (2) The nuclear flow follows the path of least resistance; that is, if one reaction rate drops by a large factor there is always another reaction capable of playing its role. (3) If the freeze-out from high temperatures is fast enough, the rates of individual reactions are much less important than the properties of nuclei (binding energy, partition function).

Second, there are narrow temperature ranges where the yields are more sensitive to the rates. For instance, the temperatures at which a modification of the rate of the

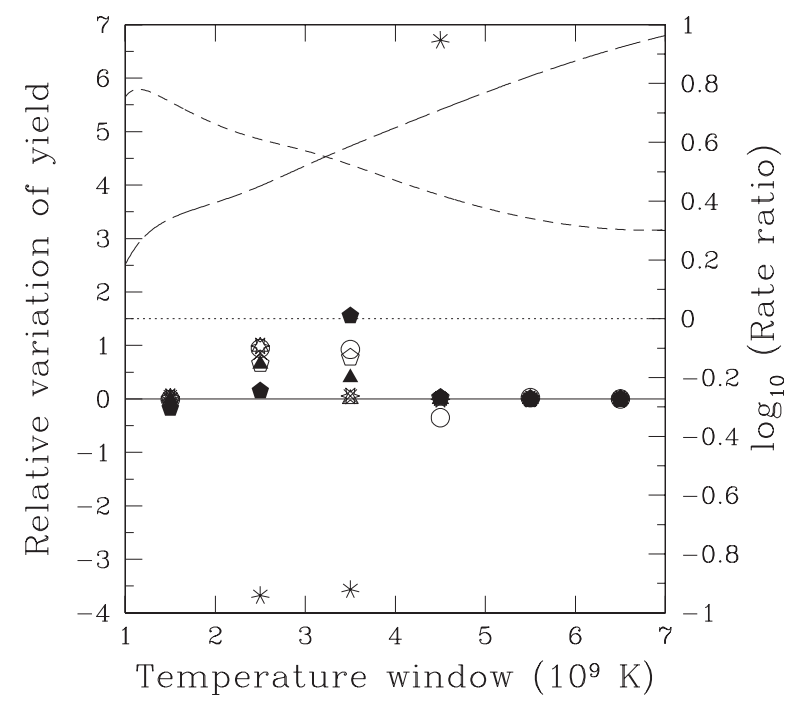

FIG. 14. Sensitivity of the yield of selected species to variations by a factor of $\times 10$ in the rate of the reactions ${ }^{20} \mathrm{Ne}+\alpha \rightleftarrows{ }^{24} \mathrm{Mg}+\gamma$ in different temperature windows of size $10^{9} \mathrm{~K}$. The meaning of the points is the same as in Fig. 13. The dashed lines (scaled according to the right axis) give the logarithm of the ratio of the different rates of the reaction ${ }^{20} \mathrm{Ne}+\alpha \rightarrow{ }^{24} \mathrm{Mg}+\gamma$ in JINA. The short-dashed line belongs to the ratio of the rate from Ref. [42] to that from Ref. [67], while the long-dashed line belongs to the ratio of the rate from Ref. [60] to that from Ref. [67]. The two horizontal lines mark the zero of the left axis, that is, no variation of the yield (solid line), and the zero of the right axis, that is, rate ratio equal to one (dotted line).

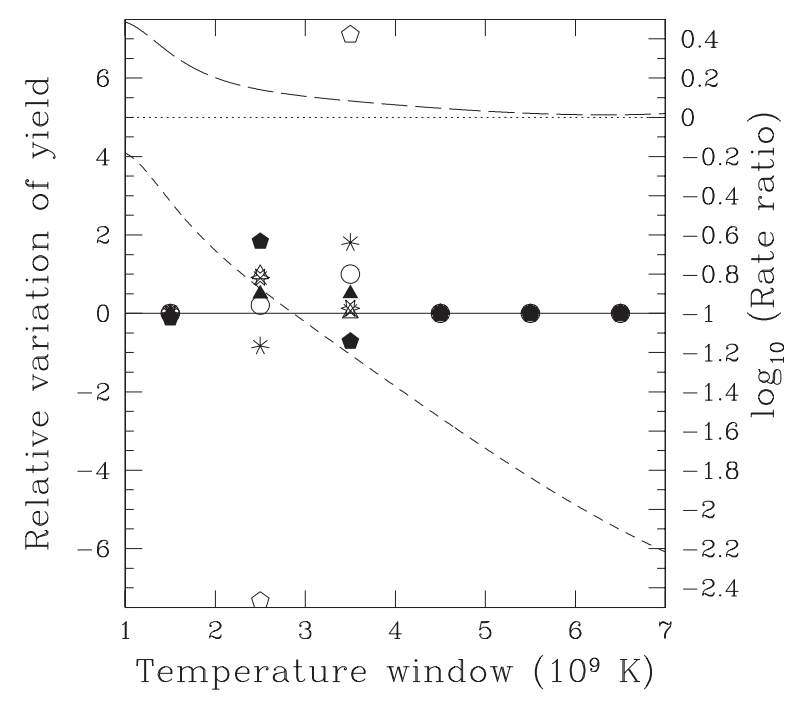

FIG. 15. Sensitivity of the yield of selected species to variations by a factor of $\times 10$ in the rate of the reactions ${ }^{24} \mathrm{Mg}+\alpha \rightleftarrows{ }^{27} \mathrm{Al}+p$ in different temperature windows of size $10^{9} \mathrm{~K}$. The meaning of the points is the same as in Fig. 13. The dashed lines (scaled according to the right axis) give the logarithm of the ratio of the different rates of the reaction ${ }^{24} \mathrm{Mg}+\alpha \rightarrow{ }^{27} \mathrm{Al}+p$ in JINA. The long-dashed line belongs to the ratio of the rate from Ref. [42] to that from Ref. [59], while the short-dashed line belongs to the ratio of the rate from Ref. [61] to that from Ref. [59]. The two horizontal lines mark the zero of the left axis, that is, no variation of the yield (solid line), and the zero of the right axis, that is, rate ratio equal to one (dotted line). 
above-mentioned three reactions has a larger impact are in the range $2 \times 10^{9} \lesssim T \lesssim 4 \times 10^{9} \mathrm{~K}$ (see Figs. 13 to 15 ). One kind of rate uncertainty we have not explored is that owing to the erroneous location of a resonance. Such a kind of error might originate an increase of the rate (with respect to the presently recommended one) in a temperature range and a decrease in a contiguous one. If this were the case, the changes of the yields of some species might be exacerbated. Thus, this kind of error in the nuclear reaction rates might be the most relevant with respect to the supernova yields.

We conclude that the explosion model chiefly determines the element production of type Ia supernovae and derived quantities such as their luminosity, while the individual nuclear reaction rates used in the simulations have a small influence on the kinetic energy and final chemical composition of the ejecta. Often it is argued that discrepancies of up to a factor of 2 between isotopic ratios in SNIa ejecta and those in the solar system, especially within the Fe-group, can be attributed to uncertainties in nuclear reaction rates. Our results show that the uncertainty in individual thermonuclear reaction rates cannot account for this factor. It remains to be seen if the yields are more sensitive to uncertainties in nuclear masses, weak interaction rates, or the simultaneous modification of the bulk of thermonuclear reaction rates. The sensitivity of the supernova nucleosynthesis to simultaneous random modifications in the bulk of thermonuclear reaction rates will be the subject of future work. In this respect, our finding is interesting in that the most influential reactions depict a clear path in a plot $Z$ vs $A$ (Fig. 12), going from ${ }^{12} \mathrm{C}$ up to ${ }^{37} \mathrm{Ar}$ through many branches involving mainly reactions with $\alpha$ particles plus the fusion reaction ${ }^{12} \mathrm{C}+{ }^{12} \mathrm{C}$. Modifications of these rates "in phase" may have interesting consequences for the chemical composition of supernova ejecta.

Finally, it is worth noting that reaction rate variations may also have an impact on the hydrostatic evolution of the progenitor of the exploding WD. Given the robustness of the explosive yields, it may well be that changes in progenitor evolution are the largest source of reaction rate sensitivity in thermonuclear supernovae.

\section{ACKNOWLEDGMENTS}

This work has been partially supported by a MEC grant, the European Union ERDF funds, and the Generalitat de Catalunya. G.M.P. is partly supported by the Deutsche Forschungsgemeinschaft through Contract No. SFB 634, the Helmholtz International Center for FAIR within the framework of the LOEWE program launched by the state of Hesse, and the Helmholtz Association through the Nuclear Astrophysics Virtual Institute (VH-VI-417).
[1] A. G. Riess, A. V. Filippenko, P. Challis, A. Clocchiatti, A. Diercks, P. M. Garnavich, R. L. Gilliland, C. J. Hogan, S. Jha, R. P. Kirshner, B. Leibundgut, M. M. Phillips, D. Reiss, B. P. Schmidt, R. A. Schommer, R. C. Smith, J. Spyromilio, C. Stubbs, N. B. Suntzeff, and J. Tonry, Astron. J. 116, 1009 (1998).

[2] S. Perlmutter et al., Nature (London) 391, 51 (1998).

[3] B. P. Schmidt et al., Astrophys. J. 507, 46 (1998).

[4] S. Perlmutter et al. and The Supernova Cosmology Project, Astrophys. J. 517, 565 (1999).

[5] A. G. Riess, P. E. Nugent, R. L. Gilliland, B. P. Schmidt, J. Tonry, M. Dickinson, R. I. Thompson, T. Budavári, S. Casertano, A. S. Evans, A. V. Filippenko, M. Livio, D. B. Sanders, A. E. Shapley, H. Spinrad, C. C. Steidel, D. Stern, J. Surace, and S. Veilleux, Astrophys. J. 560, 49 (2001).

[6] J. L. Tonry, Phys. Scr., T 117, 11 (2005).

[7] F. X. Timmes, S. E. Woosley, and T. A. Weaver, Astrophys. J. Suppl. Ser. 98, 617 (1995).

[8] F. Thielemann, D. Argast, F. Brachwitz, W. R. Hix, P. Höflich, M. Liebendörfer, G. Martinez-Pinedo, A. Mezzacappa, K. Nomoto, and I. Panov, in From Twilight to Highlight: The Physics of Supernovae, edited by W. Hillebrandt and B. Leibundgut (Springer-Verlag, Berlin, 2003), p. 331.

[9] F. Matteucci, E. Spitoni, S. Recchi, and R. Valiante, Astron. Astrophys. 501, 531 (2009).

[10] M. Stehle, P. A. Mazzali, and W. Hillebrandt, Nucl. Phys. A 758, 470 (2005)

[11] P. A. Mazzali, F. K. Röpke, S. Benetti, and W. Hillebrandt, Science 315, 825 (2007).

[12] C. Badenes, Proc. Natl. Acad. Sci. USA 107, 7141 (2010).

[13] J. Isern, E. Bravo, and A. Hirschmann, Adv. Space Res. 38, 1434 (2006).
[14] J. N. Bahcall, W. F. Huebner, S. H. Lubow, P. D. Parker, and R. K. Ulrich, Rev. Mod. Phys. 54, 767 (1982).

[15] L. M. Krauss and P. Romanelli, Astrophys. J. 358, 47 (1990).

[16] M. S. Smith, L. H. Kawano, and R. A. Malaney, Astrophys. J. Suppl. Ser. 85, 219 (1993).

[17] C. Iliadis, A. Champagne, J. José, S. Starrfield, and P. Tupper, Astrophys. J. Suppl. Ser. 142, 105 (2002).

[18] R. G. Izzard, M. Lugaro, A. I. Karakas, C. Iliadis, and M. van Raai, Astron. Astrophys. 466, 641 (2007).

[19] L. The, D. D. Clayton, L. Jin, and B. S. Meyer, Astrophys. J. 504, 500 (1998).

[20] W. R. Hix, M. S. Smith, S. Starrfield, A. Mezzacappa, and D. L. Smith, Nucl. Phys. A 718, 620 (2003).

[21] A. Arcones and G. Martínez-Pinedo, Phys. Rev. C 83, 045809 (2011).

[22] W. Hillebrandt and J. C. Niemeyer, Annu. Rev. Astron. Astrophys. 38, 191 (2000).

[23] P. Nugent, E. Baron, D. Branch, A. Fisher, and P. H. Hauschildt, Astrophys. J. 485, 812 (1997).

[24] S. E. Woosley and D. Kasen, Astrophys. J. 734, 38 (2011).

[25] E. Bravo, L. G. Althaus, E. García-Berro, and I. Domínguez, Astron. Astrophys. 526, A26 (2011).

[26] R. Napiwotzki, D. Koester, G. Nelemans, L. Yungelson, N. Christlieb, A. Renzini, D. Reimers, H. Drechsel, and B. Leibundgut, Astron. Astrophys. 386, 957 (2002).

[27] H. Saio and K. Nomoto, Astrophys. J. 500, 388 (1998).

[28] L. Segretain, G. Chabrier, and R. Mochkovitch, Astrophys. J. 481, 355 (1997).

[29] D. J. Jeffery, D. Branch, and E. Baron, arXiv:astro-ph/0609804 (2006).

[30] D. A. Howell, M. Sullivan, P. E. Nugent, R. S. Ellis, A. J. Conley, D. Le Borgne, R. G. Carlberg, J. Guy, D. Balam, 
S. Basa, D. Fouchez, I. M. Hook, E. Y. Hsiao, J. D. Neill, R. Pain, K. M. Perrett, and C. J. Pritchet, Nature (London) 443, 308 (2006).

[31] R. A. Scalzo et al., Astrophys. J. 713, 1073 (2010).

[32] M. Tanaka, K. S. Kawabata, M. Yamanaka, K. Maeda, T. Hattori, K. Aoki, K. Nomoto, M. Iye, T. Sasaki, P. A. Mazzali, and E. Pian, Astrophys. J. 714, 1209 (2010).

[33] J. M. Silverman, M. Ganeshalingam, W. Li, A. V. Filippenko, A. A. Miller, and D. Poznanski, Mon. Not. R. Astron. Soc. 410 , 585 (2011).

[34] C. Badenes, E. Bravo, and K. J. Borkowski, Adv. Space Res. 35, 987 (2005).

[35] P. Höflich, C. Gerardy, E. Linder et al., in Stellar Candles for the Extragalactic Distance Scale, edited by D. Alloin and W. Gieren, Lecture Notes in Physics Vol. 635 (Springer-Verlag, Berlin, 2003), pp. 203-227.

[36] O. Straniero, I. Domínguez, G. Imbriani, and L. Piersanti, Astrophys. J. 583, 878 (2003).

[37] P. Hoeflich, J. C. Wheeler, and F. K. Thielemann, Astrophys. J. 495, 617 (1998).

[38] F. K. Röpke, M. Gieseler, M. Reinecke, C. Travaglio, and W. Hillebrandt, Astron. Astrophys. 453, 203 (2006).

[39] A. L. Piro and L. Bildsten, Astrophys. J. 673, 1009 (2008).

[40] D. A. Chamulak, E. F. Brown, F. X. Timmes, and K. Dupczak, Astrophys. J. 677, 160 (2008).

[41] T. Rauscher, A. Heger, R. D. Hoffman, and S. E. Woosley, Astrophys. J. 576, 323 (2002).

[42] R. H. Cyburt, A. M. Amthor, R. Ferguson, Z. Meisel, K. Smith, S. Warren, A. Heger, R. D. Hoffman, T. Rauscher, A. Sakharuk, H. Schatz, F. K. Thielemann, and M. Wiescher, Astrophys. J. Suppl. Ser. 189, 240 (2010).

[43] N. Itoh, H. Totsuji, S. Ichimaru, and H. E. Dewitt, Astrophys. J. 234, 1079 (1979).

[44] E. E. Salpeter and H. M. van Horn, Astrophys. J. 155, 183 (1969).

[45] A. M. Khokhlov, E. S. Oran, and J. C. Wheeler, Astrophys. J. 478, 678 (1997).

[46] G. M. Fuller, W. A. Fowler, and M. J. Newman, Astrophys. J. Suppl. Ser. 48, 279 (1982).

[47] G. Martínez-Pinedo, K. Langanke, and D. J. Dean, Astrophys. J. Suppl. Ser. 126, 493 (2000).

[48] C. Badenes, K. J. Borkowski, and E. Bravo, Astrophys. J. 624, 198 (2005).

[49] G. Folatelli, M. M. Phillips, N. Morrell, M. Tanaka, K. Maeda, K. Nomoto, M. Stritzinger, C. R. Burns, M. Hamuy, P. Mazzali,
L. Boldt, A. Campillay, C. Contreras, S. Gonzalez, M. Roth, F. Salgado, W. L. Freedman, B. F. Madore, S. E. Persson, and N. B. Suntzeff, Astrophys. J. 745, 74 (2012).

[50] M. Tanaka, P. A. Mazzali, V. Stanishev, I. Maurer, W. E. Kerzendorf, and K. Nomoto, Mon. Not. R. Astron. Soc. 410, 1725 (2011).

[51] C. Badenes, K. J. Borkowski, J. P. Hughes, U. Hwang, and E. Bravo, Astrophys. J. 645, 1373 (2006).

[52] R. D. Hoffman, S. E. Woosley, T. A. Weaver, T. Rauscher, and F.-K. Thielemann, Astrophys. J. 521, 735 (1999).

[53] W. R. Hix and F. Thielemann, Astrophys. J. 460, 869 (1996).

[54] W. R. Hix and F. Thielemann, Astrophys. J. 511, 862 (1999).

[55] I. Dillmann, M. Heil, F. Käppeler, R. Plag, T. Rauscher, and F. Thielemann, in Capture Gamma-Ray Spectroscopy and Related Topics, edited by A. Woehr and A. Aprahamian, American Institute of Physics Conference Series Vol. 819 (American Institute of Physics, Melville, 2006), pp. 123-127.

[56] T. Rauscher and F. Thielemann, At. Data Nucl. Data Tables 75, 1 (2000).

[57] Z. Y. Bao and F. Käppeler, At. Data Nucl. Data Tables 36, 411 (1987).

[58] D. W. Bardayan, J. C. Blackmon, C. R. Brune, A. E. Champagne, A. A. Chen, J. M. Cox, T. Davinson, V. Y. Hansper, M. A. Hofstee, B. A. Johnson, R. L. Kozub, Z. Ma, P. D. Parker, D. E. Pierce, M. T. Rabban, A. C. Shotter, M. S. Smith, K. B. Swartz, D. W. Visser, and P. J. Woods, Phys. Rev. C 62, 055804 (2000).

[59] C. Iliadis, J. M. D’Auria, S. Starrfield, W. J. Thompson, and M. Wiescher, Astrophys. J. Suppl. Ser. 134, 151 (2001).

[60] C. Angulo et al., Nucl. Phys. A 656, 3 (1999).

[61] L. van Wormer, J. Goerres, C. Iliadis, M. Wiescher, and F. Thielemann, Astrophys. J. 432, 326 (1994).

[62] G. R. Caughlan and W. A. Fowler, At. Data Nucl. Data Tables 40, 283 (1988).

[63] L. Buchmann, Astrophys. J. 468, L127 (1996).

[64] R. Kunz, M. Fey, M. Jaeger, A. Mayer, J. W. Hammer, G. Staudt, S. Harissopulos, and T. Paradellis, Astrophys. J. 567, 643 (2002).

[65] A. F. Scott, A. J. Morton, S. G. Tims, V. Y. Hansper, and D. G. Sargood, Nucl. Phys. A 552, 363 (1993).

[66] S. E. Hale, A. E. Champagne, C. Iliadis, V. Y. Hansper, D. C. Powell, and J. C. Blackmon, Phys. Rev. C 70, 045802 (2004).

[67] C. Iliadis, R. Longland, A. E. Champagne, A. Coc, and R. Fitzgerald, Nucl. Phys. A 841, 31-250 (2010). 